\title{
AN OBSERVATIONAL STUDY OF PERIPHERALLY INSERTED CENTRAL CATHETER (PICC)-RELATED COMPLICATIONS AMONGST ONCOLOGY PATIENTS
}

by

\author{
Mary Fairhall
}

A thesis submitted to Victoria University of Wellington in partial fulfillment of the requirements for the

Degree of Master of Arts (Applied)

in Nursing

Victoria University of Wellington

2008 


\begin{abstract}
This thesis reports on a retrospective observational study that examined the complication rate of peripherally inserted central catheters (PICCs) within a regional cancer centre. PICCs are increasingly used for delivery of chemotherapy and other intravenous therapies in oncology patients. A literature review revealed that almost all published research on PICC complications reported on silicone (Groshong ${ }^{\mathrm{TM}}$ ) catheter use, rather than the polyurethane (Arrow ${ }^{\mathrm{TM}}$ ) PICCs used at Christchurch Hospital. Also, much literature referred to PICCs being inserted by non-nurses, whereas the Christchurch service uses specially-trained nurses to insert them. The purpose of the study was to identify the nature, incidence and rates of polyurethane (Arrow $^{\mathrm{TM}}$ ) PICC complications in an adult oncology cohort. Ethics Committee approval was gained to retrospectively follow all PICCs inserted in adult oncology patients at Christchurch Hospital over a 13-month period from 1st March 2006 until $31^{\text {st }}$ March 2007. Data collected were analysed utilising the statistical computer package SPSS. One hundred and sixty-four PICCs were inserted into 156 individual oncology patients over this period. The median dwell time was 68 days (range 6-412, IQR 39-126) for a total of 14,276 catheter-days. Complications occurred in 25 (15\%) out of 164 PICC lines, in 22 (15\%) of the 156 patients for an overall complication rate of 1.75 per 1000 catheter-days. However, only 16 of the 25 PICCs with complications required early removal $(9.75 \%$ of the cohort) for a favourably low serious complication rate of 1.12 per 1000 catheter-days. The three commonest complications were infection at $4.3 \%(7 / 164)$ or 0.49 infection complications/1000 PICC-days, PICC migration at $3 \%(5 / 164)$ or $0.35 / 1000$ catheter days, and thrombosis at $2.4 \%(4 / 164)$ or $0.28 / 1000$ catheter days. The median time to complication was 41 days (range 2-160, IQR 25-77). Those with complications were more likely to have a gastro-intestinal or an ovarian cancer diagnosis, and less likely to have colorectal cancer $(\mathrm{p}=0.001)$. These findings provide support for the safe and effective use of polyurethane (Arrow ${ }^{\mathrm{TM}}$ ) PICCs for venous access within the adult oncology context. Furthermore, it suggests that cost effective nurse-led (Arrow) PICC insertions can contribute to a low complication rate. This benchmark study should be followed by further prospective studies examining the relationship of cancer diagnosis to PICC complication rates in oncology patients.
\end{abstract}


Key words: PICC, peripherally inserted central catheter, complications, oncology, observational study, CVAD, vascular access device. 


\section{Dedication}

I wish to dedicate this thesis to my late mother, Margaret (Peg) Fairhall who with her gentle wisdom and story-telling, taught me everything I needed to know about how to be an excellent nurse. 


\section{Acknowledgements}

Completing a Masters degree is a huge undertaking for anyone, but especially so for nurses who frequently juggle work commitments, caring for growing children as well as elderly parents and extended family members, at the same time as studying. There has been a great deal of juggling of the above in the journey of this thesis and it has only been possible through the support and encouragement of some very good people along the way.

Firstly, thank you to the oncology patients I have met and nursed whose journeys have inspired me to learn and know more about oncology so I can better nurse them, and meet their complex and varied needs. Their strength and courage has motivated me through the highs and lows of thesis-writing.

To Hayley Beckman, Oncology Outpatients' Nurse and research assistant extraordinaire, without you this thesis would not have been possible. You have done an amazing job collating all the data and so willingly supporting me in this research process. You have been a tower of strength and you are an amazing nurse.

To my very good friend and mentor, Esther Vallance, who has walked this road before me and has unhesitatingly supported and encouraged me throughout this journey. Thank you for your great company, your patience and fortitude, and for your laughter and great wisdom. You have helped me see the path forward and been a rock and an inspiration when I have faltered. Your support has been immense.

To my husband Sandy, and children Hugh, Alice and Clare who have been extremely patient and encouraging whilst sharing me with this process of writing. Thank you for waiting for dinner and giving up the computer when I needed it and the many other little ways you have unselfishly supported and assisted me over the past two years.

Thank you to Dr. Kathy Nelson who has been such an understanding and wise supervisor, patiently waiting for me at times and encouraging me forward when I 
have felt bogged down. Your friendship and wisdom have eased the thesis journey, and I am enormously grateful for all your assistance.

To Doryan Mahalm, Dr. Mark Jeffery, Dr. Jane O’Malley and Helen O’Connor, thank you all for your time, assistance and wisdom.

To my Clinical Nurse Manager, Sally Braycotton, and Clinical Nurse Specialist, Sarah Ellery, thank you for your support and encouragement and for allowing me time to write.

To my very supportive employer, Canterbury District Health Board, thank you for CTA funding and for encouraging and enabling staff to undertake further learning and study whilst employees. It is greatly appreciated.

Thank you to the Thomas Tippett Trust, the Cancer Society and NERF for offering funding assistance for this project. Without your generous assistance this research would not have been possible. 


\section{Table of Contents}

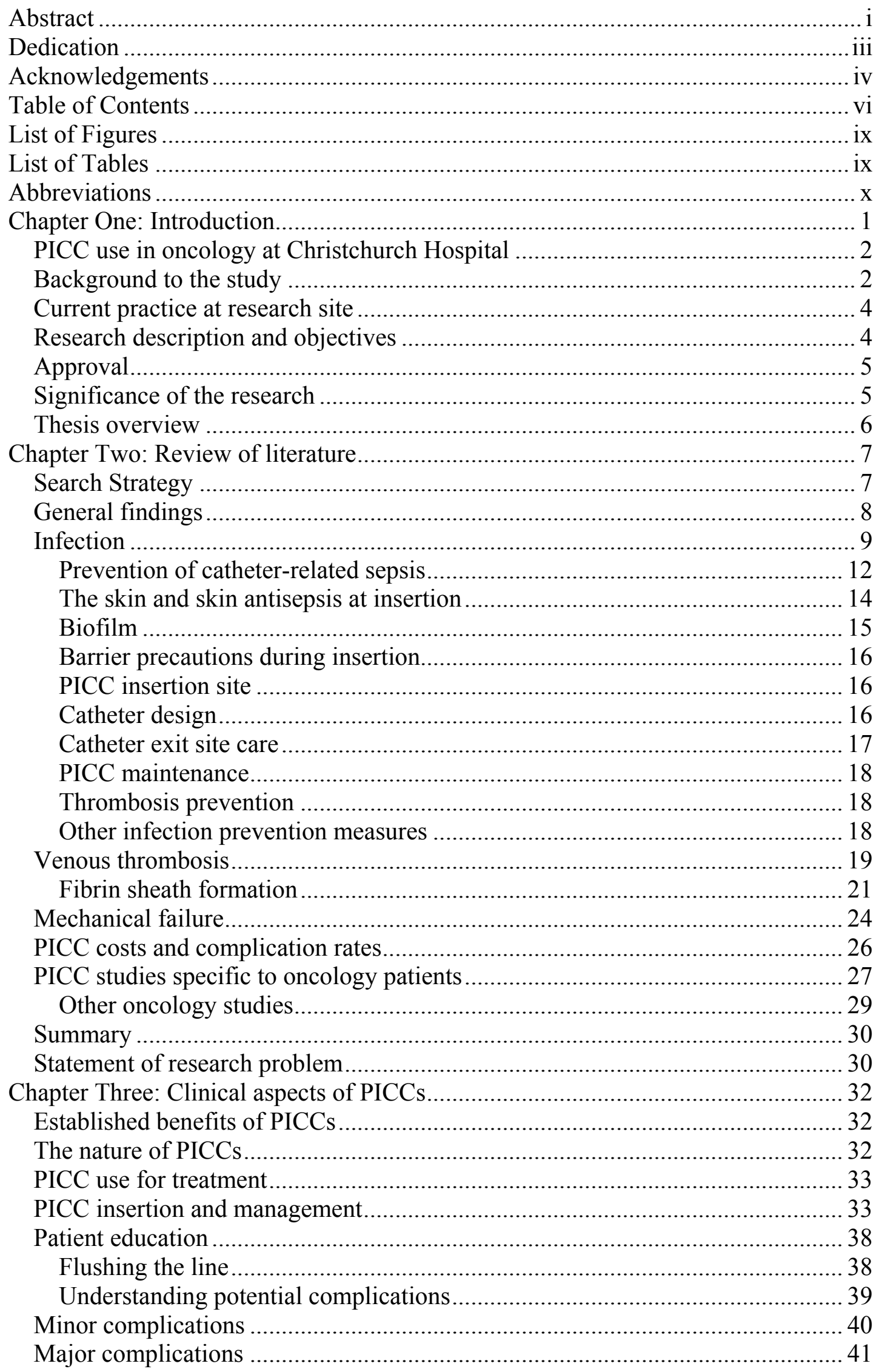




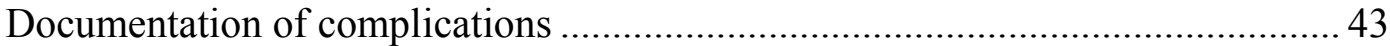

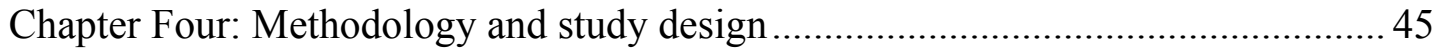

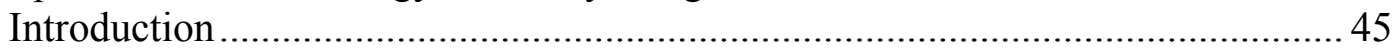

History of observational methodology and its philosophical underpinnings........ 45

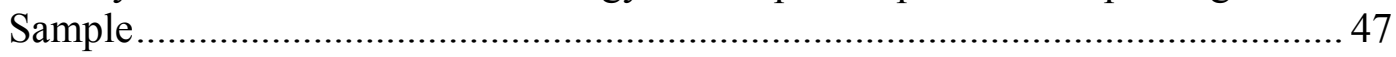

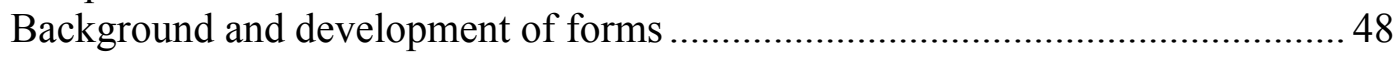

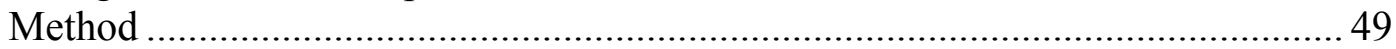

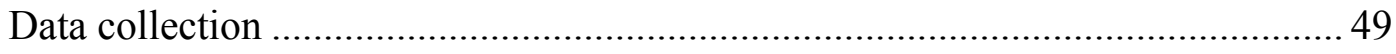

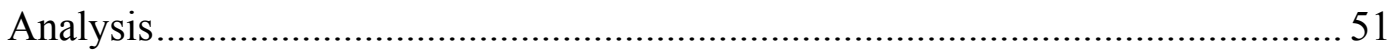

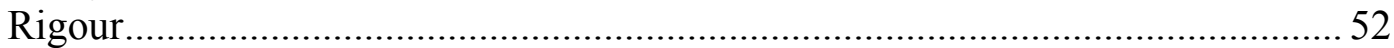

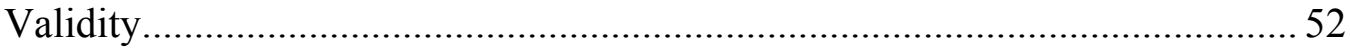

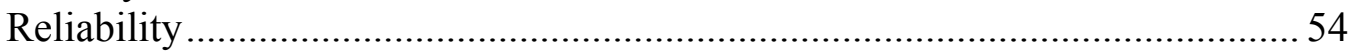

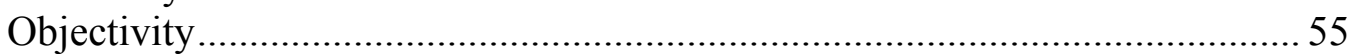

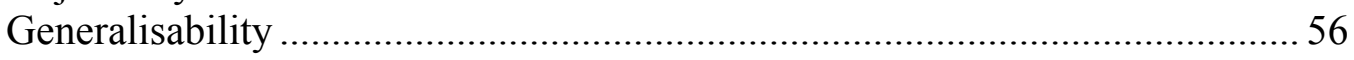

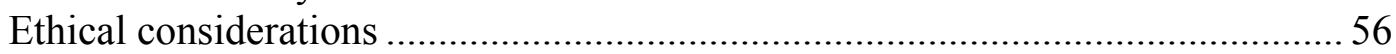

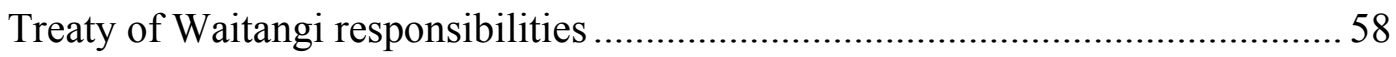

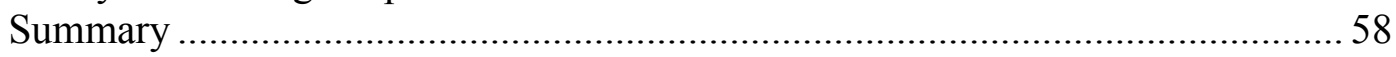

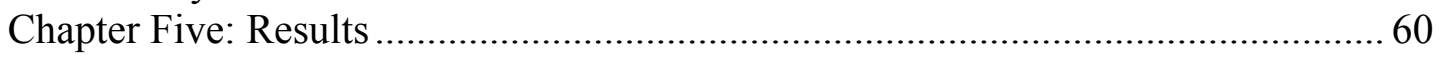

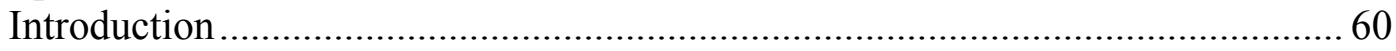

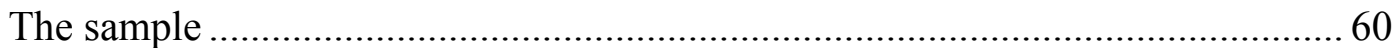

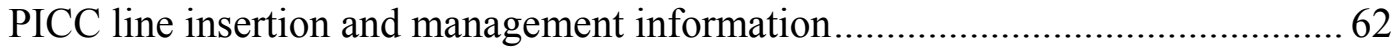

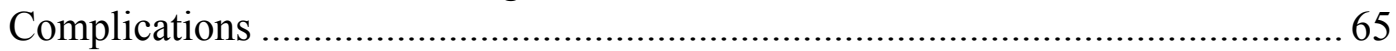

Differences between patients and PICCs with and without a complication .......... 66

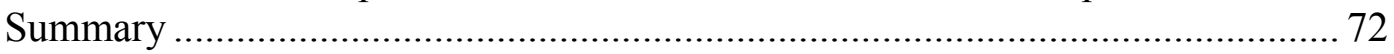

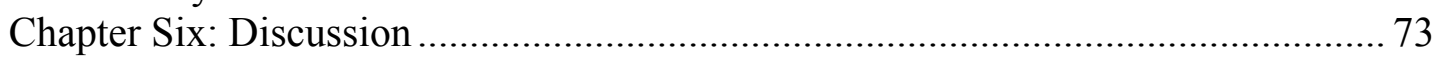

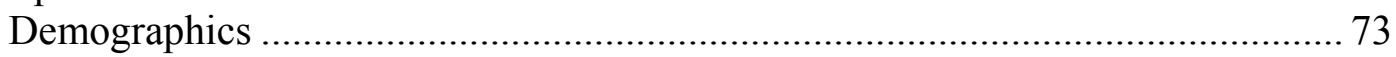

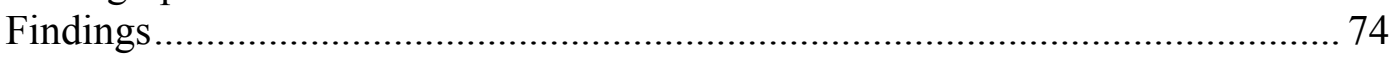

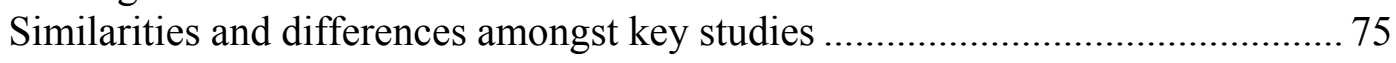

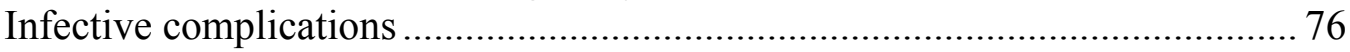

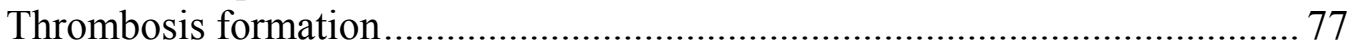

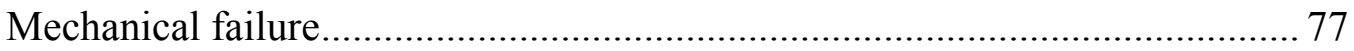

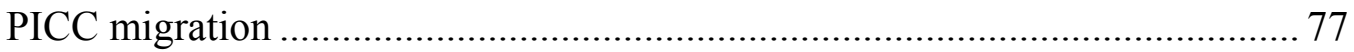

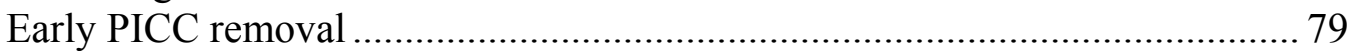

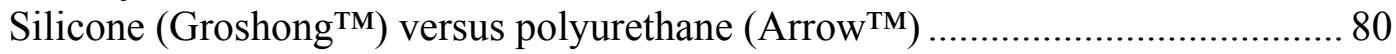

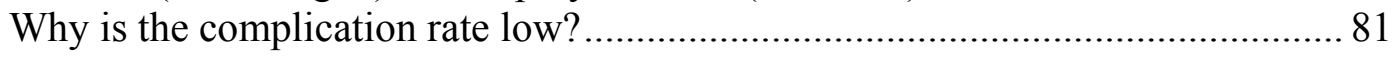

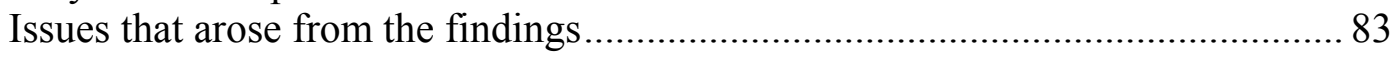

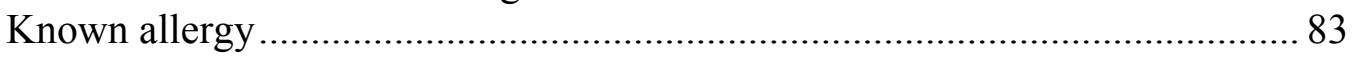

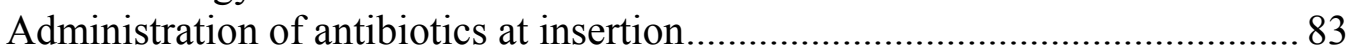

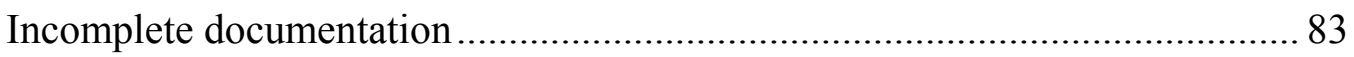

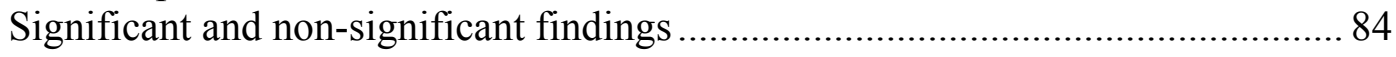

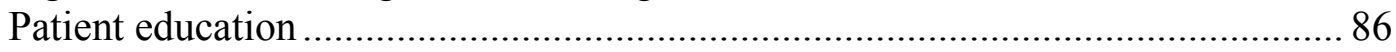

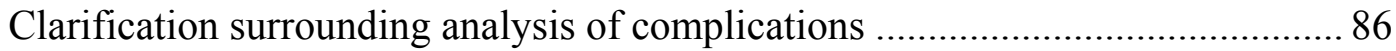

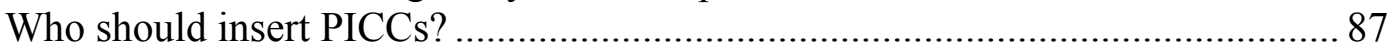

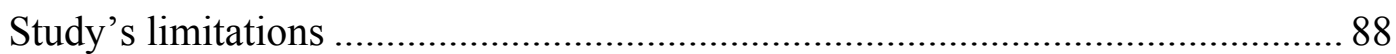

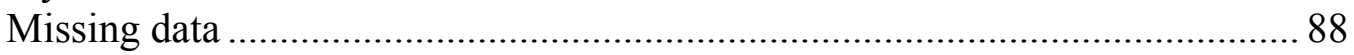

Individual variation in reporting of PICC outcomes...................................... 90

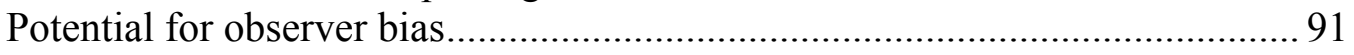

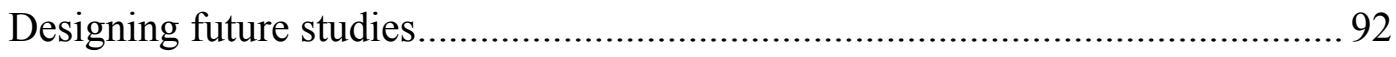

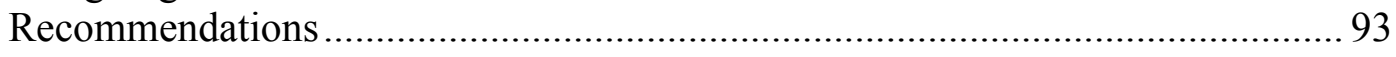

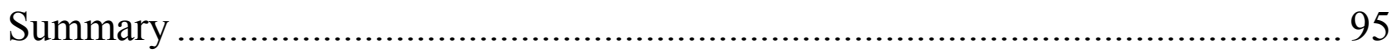




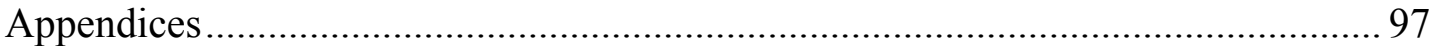

Appendix 1 - Upper-South Regional Ethics Committee Approval........................99

Appendix 2 - A Guide to your PICC/Midline: CLC2000-saline lock .................. 102

Appendix 3 - PICC/Midline Patient Information form ....................................... 113

Appendix 4 - Canterbury District Health Board Protocols for Peripherally Inserted

Central Catheters Management ......................................................................... 114

Appendix 5 - De-Clotting a PIC Catheter using the 'POP Technique' ............... 136

Appendix 6 - Maintenance and Care Plan form................................................. 138

Appendix 7 - Central Venous Access Device Record form................................ 139

Appendix 8 - Proposed Central Venous Access Device Record form................. 140

Appendix 9 - Canterbury District Health Board Approval for use of its documents

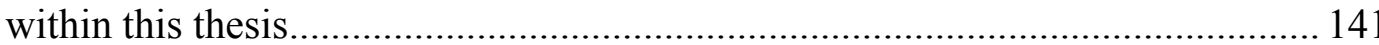

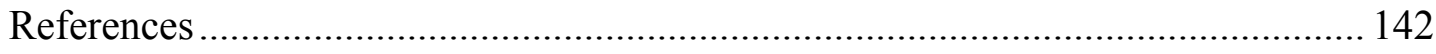




\section{List of Figures}

Figure 1: Focus for preventive strategies...................................... 14

Figure 2: All PICC complications compared over time........................... 71

Figure 3: Complications requiring PICC removal compared over time..............72

\section{List of Tables}

Table 1: Summary of PICC complications as reported in the literature

Table 2: Factors that increase the risk of venous access device-related infections...12

Table 3: Healthcare Infection Control Practices Advisory Committee (HICPAC)

Recommendations for the prevention of CRS ........................13

Table 4: Demographic and clinical features of the 156 patients with PICC lines....61

Table 5: Indicators for PICC insertion ........................................ 62

Table 6: Reasons for PICC line removal....................................64

Table 7: PICC complications by number, percent and rates......................66

Table 8: Demographic and clinical features of the 156 by presence/absence of

Complications......................................................67

Table 9: PICC details by presence/absence of complications $(n=164) \ldots \ldots \ldots \ldots \ldots . .68$

Table 10: Summary of complications encountered, treatment and PICC removal....70

Table 11: Comparison of findings with 4 key studies............................ 76 


\section{Abbreviations}

$\begin{array}{ll}\text { AIDS } & \text { Acquired Immune Deficiency Syndrome } \\ \text { CDHB } & \text { Canterbury District Health Board } \\ \text { CINAHL } & \text { Cumulative Index of Nursing and Allied Health Literature } \\ \text { CR-BSI } & \text { Catheter related blood stream infection } \\ \text { CRS } & \text { Catheter related sepsis } \\ \text { CVAD } & \text { Central Venous Access Device } \\ \text { CVA } & \text { Cerebro-vascular Accident } \\ \text { CVC } & \text { Central Venous Catheter } \\ \text { DSA } & \text { Digital Subtraction Angiography } \\ \text { DVT } & \text { Deep vein thrombosis } \\ \text { ECF } & \text { Epirubicin, Cisplatin \& Fluorouracil (chemotherapy) } \\ \text { FAS } & \text { Fibrin Analysis System } \\ \text { HICPAC } & \text { Hospital Infection Control Practices Advisory Committee } \\ \text { IV } & \text { Intravenous } \\ \text { LMWH } & \text { Low molecular-weight heparin } \\ \text { NHI } & \text { National Health Index } \\ \text { NNIS } & \text { National Nosocomial Infections Surveillance System Report } \\ \text { PICC } & \text { Peripherally Inserted Central Catheter } \\ \text { PWO } & \text { Persistent withdrawal occlusion } \\ \text { SPSS } & \text { Statistical Package for Social Sciences } \\ \text { STROBE } & \text { the Strengthening the Reporting of Observational Studies in } \\ & \text { Epidemiology Statement } \\ \text { SVC } & \text { Superior vena cava } \\ \text { tPA } & \text { Tissue plasminogen activator } \\ \text { TPN } & \text { Total parenteral nutrition } \\ \text { VAD } & \text { Venous Access Device } \\ \text { 5-FU } & \text { Fluorouracil (chemotherapy) }\end{array}$




\section{Chapter One: Introduction}

Peripherally-inserted central catheters (PICCs) are increasingly being used in oncology patients to deliver chemotherapy and other intravenous fluids and medications (Yap, Karapetis, Lerose, Iyer, \& Koczwara, 2006). PICCs first gained popularity in the United States of America in the 1980s, becoming increasingly popular because of their reduction in cost and potential complications. At Christchurch Hospital insertions were commenced in 1991 by an Intravenous Nurse Specialist in the Bone Marrow Transplant Unit at a rate of approximately 15 per month (Mahalm, Pithie, \& Chambers, 2003). Since this time, PICC use has increased substantially to the point that in 2007 specially-trained nurses inserted an average of 100 PICCs per month (Doryan Mahalm, Charge Nurse Manager, Radiology, personal communication, 19 May 2007). PICCs are invaluable in the care of oncology patients, more so because of the dynamic and changing nature of chemotherapy regimens. Hence, they have become an essential part of patient care. This study concerns itself with PICC lines and their use in oncology patients. This first chapter provides a background to the study, describes key terms, and presents an overview of the thesis.

PICCs fall within the wider category of central venous access devices (CVADs). The four major types of CVADs are non-tunnelled percutaneous central venous catheters (CVCs), tunnelled central venous catheters (Hickman lines), implanted ports (Portacaths), and PICCs (Orr \& Ryder, 1993). A PICC is defined as "a catheter inserted into a peripheral vein with the tip residing in the lower one-third of the superior vena cava" just above the right atrium (Intravenous Nurse Society, 1997, p. 172). These catheters are primarily inserted into either the basilic or brachial vein of the upper arm (Mahalm et al., 2003). PICCs are used for patients receiving vesicant or irritant chemotherapies, long term or large chemotherapy regimens, infusional chemotherapy regimens, long-term treatment with intravenous (IV) antibiotics, total parenteral nutrition, or repeated administration of blood or blood products. 


\section{PICC use in oncology at Christchurch Hospital}

This thesis reports on a retrospective observational study examining the nature of PICC complications, and their rates, within the regional cancer centre based at Christchurch Hospital. The Christchurch Hospital Oncology Service provides services for approximately 680,000 people in the South Island of New Zealand. People come from a large geographical area spanning from Nelson in the north to Timaru in the south, as well as the West Coast of the South Island. Some of these areas are sparsely populated and oncologists travel to Greymouth, Ashburton, Timaru, Nelson and Blenheim to run outlying clinics on a monthly basis. The service sees about 2,650 new patients per year and this number excludes haematological cancers. Patients with haematological cancers are assessed and treated separately through the Haematology Department and the Bone Marrow Transplant Unit of Christchurch Hospital and not the Oncology Service where this present study is situated. Of these new patients with cancer, approximately 140 patients per year are offered PICCs as the preferred method of cancer treatment delivery.

\section{Background to the study}

This study was undertaken to explore possible improvements in practice related to PICC line insertion and management in oncology patients. Oncologists and nurses anecdotally perceived a marked increase in the number of PICC complications being managed by the service and were concerned to explore this trend more specifically and scientifically. It was possible that this persistent, perceived increase in PICC complications may be a fault in PICC management or insertion, or merely related to a dramatic increase in their use, rather than an actual increase in complications per se. It was decided that an exploratory study was warranted.

PICC complications are any untoward or iatrogenic event which occurs to the patient as a result of having a PICC line in place. These complications can be divided into minor and major complications. Minor complications are complications that can usually be corrected with conservative management and do not necessitate the removal of the PICC line. They are not the focus of this study. Major complications are potentially more serious and usually result in early removal of the PICC line. The three major PICC complications are infection/sepsis, thrombosis, and mechanical 
failure, which includes catheter occlusion due to kinking, catheter migration, or fibrin sheath occlusion; leakage or broken catheter, or accidental removal (Cheong, Perry, Karapetis, \& Koczara, 2004; Mahalm, et al., 2003; Walshe, Malak, Eagan, \& Sepkowitz, 2002). A retrospective study of PICC complications within the Christchurch Hospital Oncology Department had previously been attempted but had to be aborted due to the high incidence of incomplete documentation (Ruth Gerring, Staff Nurse, Oncology, personal communication, 19 May 2007). Since the occurrence of PICC complications has the potential to significantly compromise patients' quality of life and also impair treatment options and outcomes, oncology nurses have an important role to play in minimising PICC complications. Hence, PICC complications remained an issue of concern, leading to the current study.

To understand PICC complications a literature search was undertaken. The details and critique of this search are outlined and discussed in the next chapter. The review revealed that there were two main types of PICCs, one made of silicone (Groshong ${ }^{\mathrm{TM}}$ ) and the other made of polyurethane (Arrow ${ }^{\mathrm{TM}}$ ). Most international published studies report on the silicone (Groshong ${ }^{\mathrm{TM}}$ ) catheter because it would seem it is more widely used (Renner, 1998). The main advantage of Groshong ${ }^{\text {TM }}$ catheters is that they only require flushing once a week when not in use. In contrast, the polyurethane (Arrow ${ }^{\mathrm{TM}}$ ) catheter requires daily flushing when not used. At Christchurch Hospital, the site at which this research study was conducted, the polyurethane (Arrow ${ }^{\mathrm{TM}}$ ) PICC is the catheter of choice. However, an extensive search of the literature, whilst revealing several laboratory-based studies, demonstrated a scarcity of published clinical studies to confirm this. Only one local unpublished study (Mahalm et al., 2003) was located which had researched complications in polyurethane PICCs and this was in a general hospital population. Thus, there emerged a gap in the literature with regards to polyurethane PICC complications. It was also noted that much of the literature refers to PICCs being inserted by non-nurses, mostly radiologists. However the Christchurch Hospital service has a team of specially-trained nurses to insert the PICCs, with radiological back-up for difficult insertions. Furthermore only a few studies were found on PICC complications relating specifically to oncology patients. 
The reason polyurethane (Arrow ${ }^{\mathrm{TM}}$ ) PICCs were used at Christchurch Hospital was two-fold. First, laboratory-based scientific literature suggested that polyurethane catheters developed fewer complications than silicone catheters (Angle et al., 1997; Mayo, Helsabeck, \& Horne, 1995; Renner, 1998; Sheretz et al., 1995). Secondly, silicone PIC catheters had previously been trialled in the Haematology Department of Christchurch Hospital. Staff experienced difficulty inserting the silicone catheters and had trouble repairing them when the catheters failed (Doryan Mahalm, Charge Nurse Manager, Radiology, personal communication, 1 February 2008). So a change was made to polyurethane (Arrow ${ }^{\mathrm{TM}}$ ) catheters, with anecdotal evidence of improved success.

\section{Current practice at research site}

All PICCs are inserted by a dedicated team of specially-trained nurses in Digital Subtraction Angiography, the interventional suite of the Radiology Department at Christchurch Hospital, with radiologist back-up for difficult insertions. These nurses currently insert about 100 PICCs per month. Those inserted into oncology patients are primarily managed by oncology nurses for cancer treatments, most often chemotherapy regimens. Either the oncology nurses or district nurses change the PICC dressing every seven days and monitor the PICC line site for any complications. A major advantage for the oncology patient with a PICC in situ is that their chemotherapy treatment can be continued as an outpatient rather than as an inpatient. Nurses educate the patient or a relative about how to safely flush the PICC at home. However, on occasions, major PICC complications do occur which may necessitate hospitalisation and/or premature removal of the PICC.

\section{Research description and objectives}

The objectives of this retrospective observational research study were to identify and describe PICC complications and their rates, in a cohort of oncology patients.

Data were retrospectively accessed from medical records and were analysed by using the Statistical Package for Social Sciences (SPSS) version 14. The current study sought to identify and describe the complications that occurred whilst the PICC was in situ, as well as the rates of those complications. Tentative relationships between emergent variables, for example, differences between patients that had complications 
and those that did not, were also of interest to the researcher. The research question became: what are the types and rates of polyurethane (Arrow ${ }^{\mathrm{TM}}$ ) PICC-related complications in an oncology patient cohort over a 13-month period at Christchurch Hospital?

\section{Approval}

Ethics approval for this study was applied for in November 2006 and granted in February 2007 by the Upper-South Regional Ethics Committee (Appendix 1).

\section{Significance of the research}

The expressed intention of this study was to contribute to the knowledge of PICC use in oncology patients. This study is important for three reasons: it provides an understanding about the type of complications and the complication rate of polyurethane (Arrow ${ }^{\mathrm{TM}}$ ) catheters in oncology patients and attempts to compare its findings with the literature; it provides evidence for the role of nurses in inserting and managing PICC lines; and it informs ongoing service improvement, through the findings and recommendations. By documenting the types and rates of complications, it enables services to look at how complications might be minimised or ideally, prevented. This study also contributes data for the Christchurch Hospital service when it makes decisions surrounding use of polyurethane (Arrow ${ }^{\mathrm{TM}}$ ) PICCs. Formulating a research study that could be easily replicated was also a stated objective.

According to von Elm et al. (2007, p. 867) "the credibility of research depends on a critical assessment by others of the strengths and weaknesses in study design, conduct and analysis". To this end, von Elm et al. developed guidelines for reporting observational studies called STROBE (The Strengthening the Reporting of Observational Studies in Epidemiology Statement). Their checklist of 22 items that inform these guidelines were utilised to guide the description, the methodology, and the findings of this study. 


\section{Thesis overview}

This thesis presents the findings of a retrospective observational study that examined complications and their rates in polyurethane (Arrow ${ }^{\mathrm{TM}}$ ) PICC lines within a regional cancer centre. This introductory chapter has provided a background to the study, described key terms and presented an overview of the thesis.

In Chapter 2 the literature pertaining to PICC complications specifically and CVADrelated complications generally, are presented. Four specific studies on PICC complications in oncology patients are discussed in more depth, in order to establish the rationale for the design of this study.

Chapter 3 details the clinical setting for this study and describes in detail how PICCs are inserted and managed within the Christchurch Hospital service.

Chapter 4 presents the observational research methodology used for this study and discusses its retrospective design. The sample, data collection method, data analysis and the steps taken to ensure validity and reliability are described. Ethical considerations are also outlined.

Chapter 5 presents the research findings utilising the Statistical Package for the Social Sciences (SPSS), version 14. The results are described, and further presented in tables.

Chapter 6 discusses the findings in relation to the literature and clinical practice. Explanations are given for expected as well as unexpected findings. The research design process and limitations are also discussed, as are implications and recommendations for nursing practice. Suggestions are made so that similar studies can be successfully replicated by others in the oncology setting. 


\section{Chapter Two: Review of literature}

Peripherally-inserted central catheters (PICCs) offer certain advantages over other central venous access devices (CVADs) but despite their widespread use, few studies have focused on PICCs in patients with cancer. In this chapter the findings of a nursing and multidisciplinary literature review relating to PICC-related complications specifically, and CVAD-related complications generally, are presented. The literature presented is comprehensive, covering both complications as well as interventions that minimise complications. As there are only a small number of published studies on PICC lines in the oncology setting, the wider body of knowledge about central venous access devices (CVADs) in other patient population groups is encompassed. The rationale for, and design of this study, emerged from these research findings, and the current body of knowledge available on PICCs and their complications in oncology patients is presented.

\section{Search Strategy}

A search of the online databases including the Cumulative Index of Nursing and Allied Health Literature (CINAHL), Medline, PubMed, Medscape, Cochrane and Blackwell Synergy, was executed using the following search terms: peripherally inserted central catheter or PICC or central venous access device or CVAD or vascular access device or VAD and complications and/or oncology or cancer. In a second search, the following search terms were added: and/or infection or sepsis and/or thrombosis or thrombus and/or mechanical failure. The search strategies initially identified 2149 articles. The search was narrowed to 1170 articles when 'paediatric' or 'pediatric' patient studies were excluded, and further narrowed to 215 when 'renal', 'dialysis', 'urology', and 'intrathecal' and any duplicates were excluded. They were ordered by relevancy. The title and abstract screening of the 215 unique citations identified 140 as potentially eligible for review. The full text screening of the 140 citations identified 119 potentially eligible publications. Several excellent articles were also identified from the reference lists in published research. The Oncology Clinical Nurse Specialist, the Gynaecology Clinical Nurse Specialist, the Haematology Research Nurse, and the Radiology Charge Nurse Manager were also asked for any relevant articles they had on the topic which uncovered at least 20 
other relevant, older articles. Initially only journal articles from $1^{\text {st }}$ January 1995 to $31^{\text {st }}$ December 2007 were accessed as it was during this time period that PICCs were in routine use at Christchurch Hospital, but it quickly became clear that some earlier articles were critical to understanding the current body of knowledge, so these key articles were also included in the literature search. The research accessed was both national and international including studies based in the United States of America, Canada, United Kingdom, Australia, and Holland.

\section{General findings}

The literature search produced four main publication types; literature reviews, clinical guidelines, laboratory studies and clinical research. The review's findings are categorised into five main sections. The first three are; infective complications, thrombosis, and mechanical failure. Section four provides information surrounding cost effectiveness of PICCs and their complications compared with other CVADs. The fifth section represents literature on CVAD and PICC complications specifically in oncology patients.

When reviewing the literature on PICC complications, it became clear that most published research studies on PICC complication rates used silicone (Groshong ${ }^{\mathrm{TM}}$ ) catheters (Cardella, Cardella, Bacci, Fox, \& Post, 1996; Chemaly et al., 2002; Eastridge \& Lefor, 1995; Goodwin \& Carlson, 1993; Macklin \& Chernecky, 1997; Mayo et al., 1995; Pasquale, Campbell, \& Magnant, 1992; Schmid, 2000; Smith, Friedell, Cheatham, Martin, Cohen, \& Horowitz, 1998; Todd, 1998; Walshe et al., 2002), and that little is known about the complication rates with polyurethane $\left(\right.$ Arrow $\left.^{\mathrm{TM}}\right)$ catheters.

Furthermore literature, though scant, does exist that suggests complication rates with PICCs is higher in oncology patients compared with the general population probably due to immuno-compromise and prothrombotic tendency (Cheong et al., 2004; Last, Mansi, Oakley, \& Lofts, 1998; Smith et al., 1998; Walshe et al., 2002; Yap et al., 2006). However, the convenience of the PICC device is so highly rated by oncology patients that authors consider the risks worth taking (Chernecky, 2001; Mahalm et al., 2003; Walshe et al.). 
Potential complications are inherent in any device that is inserted into the body, and more specifically directly into the bloodstream. Several potential/actual complications were revealed in the literature, the rates of which were varied. For example, complication rates requiring PICC removal varied from 23.6\% (Mahalm et al., 2003) in the general hospital population to as high as $40.7 \%$ in a group of oncology patients with solid tumours (Cheong et al., 2004). Although rates of complications varied, three major complications did emerge from the literature, namely infection/sepsis, venous thrombosis, and mechanical failure, and these are now discussed.

To introduce the literature review on complications, the causes of infection, thrombosis and mechanical failure in PICCs are summarised in Table 1. Following this table, each major complication of PICCs is discussed in depth.

\section{Table 1: Summary of PICC complications as reported in the literature}

\begin{tabular}{|c|c|c|}
\hline $\begin{array}{ll}\text { Infection } \\
\text { Higher with: } \\
\text { - } & \text { oncology patients, esp. } \\
\text { neutropenic patients } \\
\text { - } & \text { poor nutritional status } \\
\text { - } & \text { multiple catheter } \\
\text { - } & \text { multiple lumens } \\
\text { - } & \text { poor insertion technique } \\
\text { - } & \text { poor PICC maintenance } \\
\text { - } & \text { technique } \\
\text { - } & \text { presence of biofilm } \\
\text { - } & \text { lipid infusion or TPN } \\
\text { - } & \text { hub contamination } \\
\text { - } & \text { silicone catheters } \\
\text { - } & \text { longer dwell time }\end{array}$ & 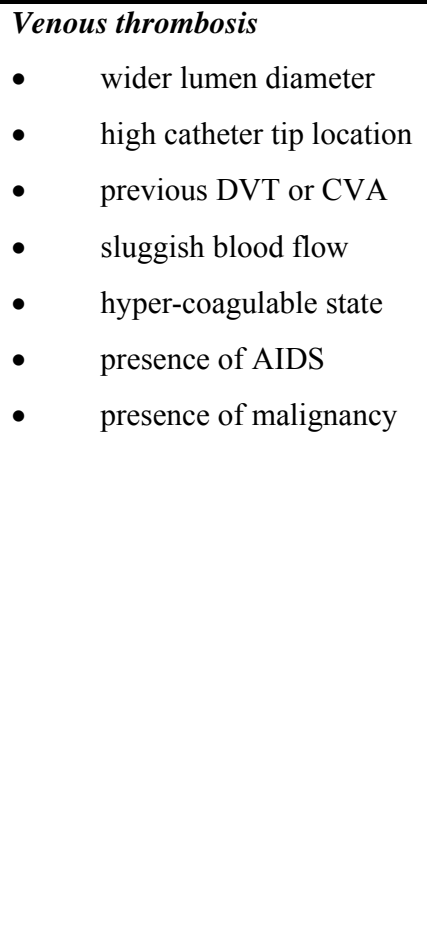 & $\begin{array}{l}\text { Mechanical failure } \\
\text { - } \quad \text { catheter tip displacement } \\
\text { - } \quad \text { catheter migration } \\
\text { - } \quad \text { catheter malposition } \\
\text { - } \quad \text { excessive pressure when } \\
\text { flushing } \\
\text { - } \quad \text { inadequate catheter } \\
\text { construction } \\
\text { fibrin sheath or tail } \\
\text { formation } \\
\text { - } \text { lipid occlusion } \\
\text { kinking of catheter } \\
\text { silicone versus } \\
\text { polyurethane catheter } \\
\text { construction }\end{array}$ \\
\hline
\end{tabular}

\section{Infection}

The most common CVAD complication is infection or sepsis and its presence is a 
major hazard for patients with PICCs. This is particularly problematic for immunocompromised oncology patients, and even more so those with neutropenia (Moran \& Camp-Sorrell, 2002; Rotstein, Brock, \& Roberts, 1995). Although PICCs generally have been associated with lower infection rates compared with other non-tunnelled CVCs (Mahalm et al., 2003; Moran \& Camp-Sorrell, 2002; Ng, Ault, Ellrodt, \& Maldonado, 1997), PICC line infections do occur. The incidence of infection varies between oncology and non-oncology patients. Schmid (2000), when reporting on a number of studies, described a range of incidences of between zero and $7 \%$ bloodstream infections in general hospital patients and Mahalm and colleagues reported an incidence of $9.2 \%$ confirmed or suspected infections out of 660 polyurethane PICCs inserted into general hospital patients.

In comparison, in a prospective study of oncology patients with PICC lines, Walshe et al. (2002) described an incidence of 7.4\% reported infections (2.5 per 1000 catheter-days) as compared with Eastridge and Lefor (1995) who reported 10\%. Furthermore, Cheong et al. (2004) reported a rate of $25.7 \%$ in a group of oncology patients with solid tumours. A possible confounding factor in oncology patients is their predisposition to infection due to being immuno-suppressed from chemotherapy and/or their disease process. This is especially true of patients with haematological malignancies, or those undergoing high dose therapies with stem cell rescue. These patients are not part of this study's cohort. Some studies have suggested that multilumen catheters were associated with a greater risk of infection than were single lumen catheters (Yeung, May, \& Hughes, 1988; Pemberton, Lyman, Lander, \& Covinsky, 1986). This may be due to increased trauma at the insertion site because of the larger catheter size and/or because multiple lumens increase the frequency of CVAD manipulations. At least two studies have identified that multiple catheter manipulations significantly increased the risk of infection (Eastridge \& Lefor, 1995; Schmid, 2000).

These infections cause significant morbidity, diagnostic uncertainty, delays in cancer therapy and occasionally, mortality (Moran \& Camp-Sorrell, 2002). Episodes of infection prolong hospitalisation by seven days or more (Pittet, Tarara, \& Wenzel, 1994) and expose the patient to expensive and potentially toxic antimicrobial and antifungal agents. Frequently these infections require catheter removal and 
sometimes catheters may be unnecessarily removed for suspected, but unconfirmed sepsis (Sanders, 2006).

Having a catheter related blood stream infection (CR-BSI), more commonly known as catheter-related sepsis (CRS), can have a significant economic impact on a hospital service with described costs ranging from US\$10,000 to $\$ 35,000$ (Donowitz, Maki, Crnich, Pappas, \& Rolston, 2001). There is also a significant personal cost for the individual and their family, for example, by loss of earnings and travel costs. Rickard (2003) also describes the human cost, such as stress and anxiety which is difficult to quantify. Alongside these considerations, any mortality has huge personal and economic impacts on families and societies (Pittet et al., 1994). Hence prevention of catheter-related sepsis is important if costs are not to outweigh the benefits of PICC access. Several factors can increase the risk of catheter related sepsis (CRS), including patient factors, catheter factors, and caregiver factors (Moran \& Camp-Sorrell, 2002). Table 2 provides a combined list of these factors identified from the literature. The strength of the evidence for each of these factors varies, and the extent of each factor's impact has not been assessed. 
Table 2: Factors that increase the risk of venous access device-related infection

\section{Patient Factors}

- Decreased immune system

- Presence of infection at the time of venous access device (VAD) insertion

- Presence of fibrin sheath, biofilm or thrombus in or around VAD

- Lack of education to assess for signs of complications

- Poor VAD maintenance

- Poor nutritional status

- Elderly or very young

- Loss of skin integrity

- Antibiotic therapy

\section{Catheter Factors}

- Polyvinyl chloride or silicone material

- Insertion site in chest or groin

- Type of catheter (non-tunnelled vs. implanted port)

- Multiple catheter lumens

\section{Caregiver Factors}

- Poor aseptic technique during catheter insertion

- Poor aseptic technique during routine maintenance

- Lack of education to assess for signs of infection

- Poor hand-washing technique

\section{Prevention of catheter-related sepsis}

Guidelines have been published and updated (O'Grady et al., 2002c) for the prevention of CRS by the USA based but world-respected Hospital Infection Control Practices Advisory Committee (HICPAC). See Table 3 for a full description of these recommendations. 


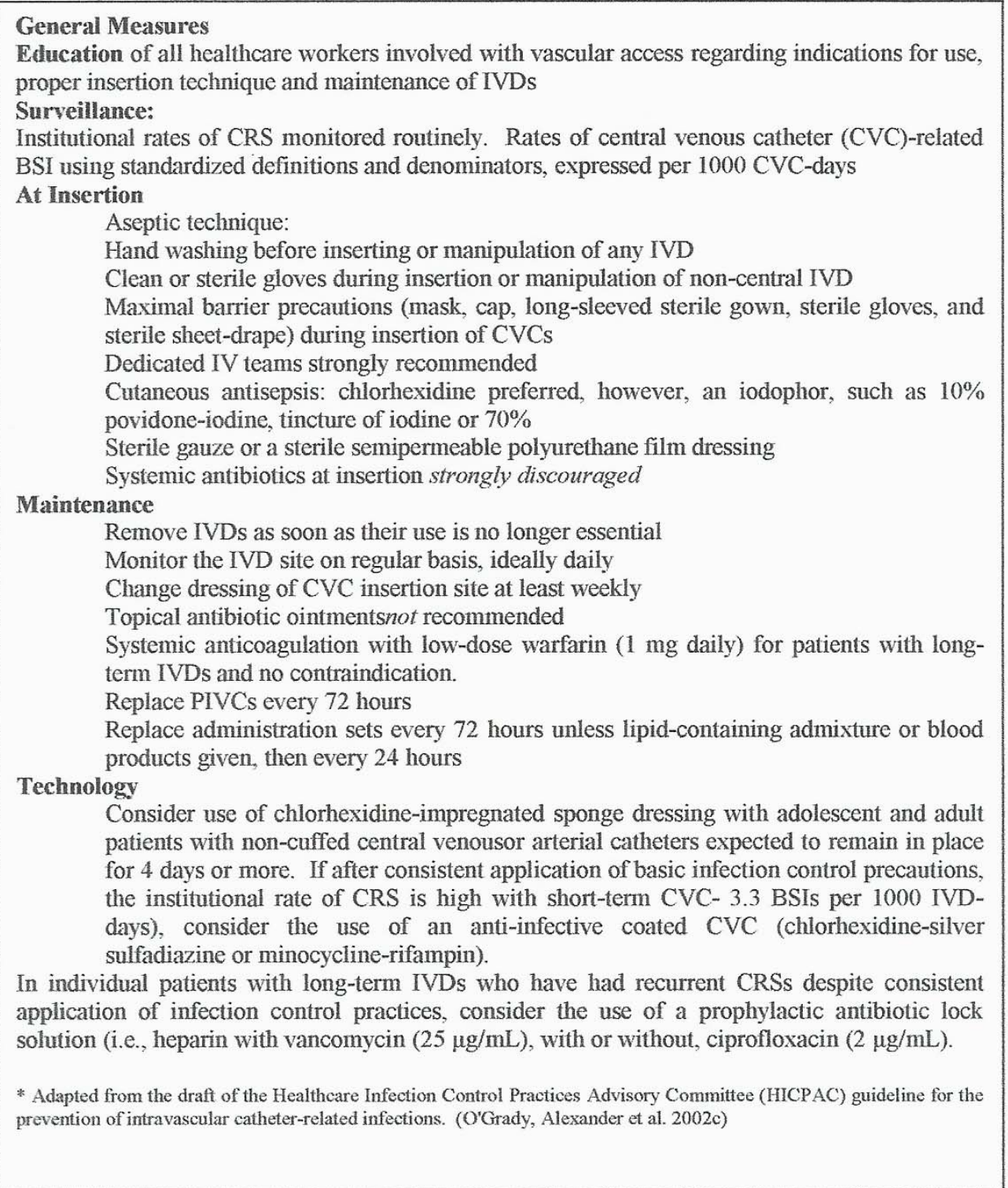

Source: Sanders, J. (2006). A prospective double blind randomised clinical trial of 70 percent ethanol to prevent catheter related sepsis in tunnelled catheters in haematology patients treated with chemotherapy. p.8 (Reproduced with permission of author)

With the introduction of standardised guidelines, the USA based National

Nosocomial Infections Surveillance System Report (NNIS) has described a reduction

in CRS, but this incidence needs to be further reduced in order to benefit immunocompromised and neutropenic oncology patients. Figure 1 reproduces a summary of the different areas that have been the foci for preventive strategies (Mermel, 2000; Sanders, 2006). These are discussed further in the following section. 


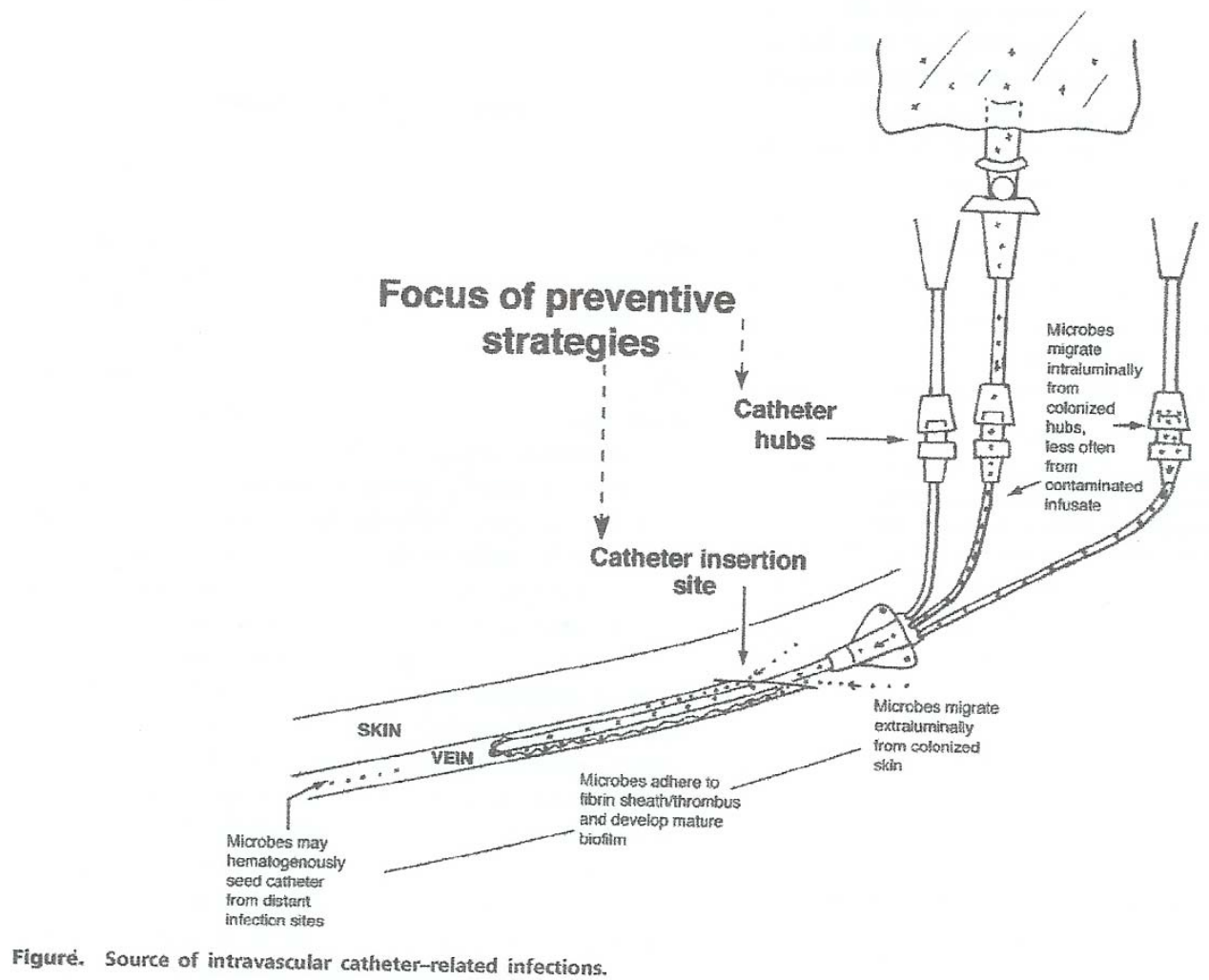

Figure 1: Focus for preventive strategies

Source: Mermel, L. A. (2000). Prevention of intravascular catheter-related infections. Annals of Internal Medicine, 132(5), p.392 (Reproduced with permission of Annals of Internal Medicine)

\section{The skin and skin antisepsis at insertion}

The skin is normally a good barrier to micro-organisms. Once this cutaneous barrier is broken at PICC insertion however, normal skin organisms such as Staphylococcus aureus and S. epidermis (Maki, Bennett, \& Bractiman, 1992) can cross the skin barrier into the catheter and/or bloodstream. There is now strong evidence that normal skin flora at the PICC insertion site are a major risk factor for the development of CRS (Rickard, 2003). Therefore, disinfection of the skin prior to PICC insertion and during follow-up care is a very important infection prevention strategy. According to Maki, Ringer, and Alvarado (1991), it is equally important that the cleansing agent of choice has been tested in randomised controlled clinical trials. They assessed the efficacy of three antiseptic solutions for skin antisepsis at catheter insertion site: $70 \%$ alcohol, $10 \%$ povidone-iodine, and aqueous solution of $2 \%$ chlorhexidine gluconate. The $2 \%$ chlorhexidine treatment was associated with the lowest rate of device-related infection ( 2.3 per 100 catheter days vs. 7.1 for the povidone-iodine and 9.3 for the alcohol, $p=0.02$ ). Only one out of the 14 infusion- 
related bacteraemias occurred from the chlorhexidine group leading the authors to conclude that the $2 \%$ chlorhexidine gluconate solution could substantially reduce the incidence of device-related infection. Mimoz et al. (1996) carried out a similar study, finding that the chlorhexidine gluconate antiseptic was significantly superior in preventing gram-positive bacterial infections but not gram-negative infections.

Two other external potential sources of infection are contamination of the infusate (Maki \& Mermel, 1998) and touch contamination of the PICC hub (Maki, 1991). Infusate contamination is relatively uncommon although total parenteral nutrition (TPN) and lipid emulsions provide a better medium for bacterial and fungal growth. For this reason, their administration time should not exceed 12 hours (Orr \& Ryder, 1993). Touch contamination of the hub by health personnel accessing the PICC has been described as a common source of catheter-related sepsis. It has been estimated to occur in up to $22 \%$ of infusion systems and is a good predictor of catheter sepsis (Maki \& Ringer, 1987). Rigorous hand-washing with 2\% chlorhexidine gluconate wash and non-touch, aseptic technique whenever the PICC is accessed, have been found to improve these statistics (O'Grady et al., 2002a).

\section{Biofilm}

The skin insertion site is a major source of contamination of CVADs within 24 hours of insertion. It has been a more recent discovery that organisms may become embedded in a polysaccharide matrix of biofilm produced by the micro-organisms that stick to the inside lumen of the catheter. These microbes may also be free to move about unattached to the biofilm (free living) (Mack, 1999). Once the microbes are attached within the biofilm, because they grow at such a slow rate, they are difficult to remove and are impervious to the body's own immune systems, such as macrophages and other phagocytes (Rickard, 2003). Thus they can live using the surrounding nutrients without hindrance from the immune system or antibiotic therapy. Micro-organisms can be released from the biofilm, seeding into the patient's bloodstream, causing an infection. The biofilm's antimicrobial resistance is a major concern as it has been estimated that $65 \%$ of nosocomial infections can be attributed to biofilms costing more than a billion US dollars per annum (Archibald \& Gaynes, 1997; Costerton, 1995; Potera, 1999). 
One solution to overcoming this biofilm without expensive anti-microbial therapy is the use of the Fibrin Analysis System (FAS) Brush (FAS Medical, Middlesex, England). This endoluminal brush cleans the inside of the catheter removing the biofilm. To date results with this brush have been mixed (Sanders, 2006; Tighe, Kite, Fawley, Thomas, \& McMahon, 1996). In spite of all these preventive measures, infection still occurs.

\section{Barrier precautions during insertion}

Barrier precautions undertaken during insertion can range from sterile gloves and drapes only, to surgical asepsis including sterile gloves, drapes, long-sleeved gown with surgical mask and cap. Studies show that treatment centres that follow full aseptic precautions during CVAD insertion have significantly lower rates of CRS compared to those that do not (Abi-Said et al., 1999; Maki, 1994). It has also been shown that having the same team of dedicated specialists to insert the catheters does reduce the rate of CRS. In a randomised trial CVADs inserted by these specialists had $0-0.2$ catheter infections per 100 catheter days as compared to lines inserted by others (1.5-2.1 catheter infections per 100 catheter days) (Maki, 1994; Sanders, 2006; Tomford, Hershey, McLaren, Porter, \& Cohen, 1984).

\section{PICC insertion site}

It has been established that the site at which CVADs are inserted influences the risk rate of CRS. Duerksen, Papineau, Siemens, and Yaffe (1999) showed a lower rate of CRS in PICC lines compared to other tunnelled and non tunnelled catheters. The risk of infection from PICCs is decreased because fewer organisms live on the arm compared to those on the chest and neck, and because the area is some distance away from the nose and mouth (Moran \& Camp-Sorrell, 2002).

\section{Catheter design}

The material used to manufacture PICCs has been associated with a variable incidence of infection. Sheretz et al. (1995) found that silicone catheters had a greater risk of infection and colonisation of organisms on the catheter than polyurethane and Teflon catheters. Similarly, Sheth et al. (1983) showed that the colonisation rates of 
polyvinyl chloride or polyethylene catheters had a higher incidence than catheters made of Teflon or polyurethane.

Antimicrobial-impregnated or antimicrobial-coated CVADs have been an important addition to the group of preventive strategies (Mermel, 2000). A meta-analysis of studies testing the efficacy of chlorhexidine and silver sulfadiazine impregnated catheters demonstrated that their short term use reduced the risk for CRS (Veenstra, Saint, Saha, Lumley, \& Sullivan, 1999). In another meta-analysis of 11 studies, Marin, Lee, and Skurnick (2000) showed that a combination of anti-microbial impregnated and heparin-bonded CVADs reduced infection by an average of $2.32 \%$. Standard use of these antimicrobial-coated catheters has not eventuated within oncology populations to date, due to concern for antibiotic resistance over time and the fact that the antimicrobial coating appears to break down after a few weeks. They may have a place for at risk neutropenic patients requiring short-term CVAD use. O'Grady et al. (2002b) recommend use of anti-infective coated CVADs when the institutional rate of CRS is high despite consistent application of basic infection control precautions.

There is also evidence to suggest that a smooth surface on the CVAD will reduce infection (Raad et al., 1993; Tebbs, Sawyer, \& Elliot, 1994). Tebbs et al. showed that the smoother the CVAD, the less likely it is that microbes will form a biofilm.

\section{Catheter exit site care}

When changing the dressing, practice has evolved through best practice guidelines (O'Grady et al, 2002a) to cleaning the site with chlorhexidine $2 \%$ cleansing solution with moderate friction in a grid-like motion (Doryan Mahalm, Charge Nurse Manager, Radiology, personal communication, 10 March 2008) however at the time the current study was undertaken the practice entailed cleaning with gentle friction with a circular, spiral motion from the exit-site outwards. Randomised controlled trials comparing gauze dressings and semi-permeable polyurethane dressings showed no difference between the two groups in relation to the rate of catheter site colonisation (Hoffmann, Weber, Samsa, \& Rutala, 1992; Maki \& Mermel, 1997; Maki, Stolz, Wheeler, \& Mermel, 1994). However, a local published study 
(Chambers et al., 2005) found that the use of a transparent semi-permeable polyurethane dressing in combination with a slow release chlorhexidine gluconate patch (Biopatch) was very effective in reducing exit site and tunnel infections. In the Chambers et al. study of neutropenic patients, only $9 \%$ (5/58 catheters) of the chlorhexidine patch group developed exit site/tunnel infections compared with $43 \%$ (23/54) of those in the control group (with no Biopatch).

\section{PICC maintenance}

The more frequently the PICC is accessed the higher the risk for contamination (Sanders, 2006). The surface of the hub should be disinfected prior to each time it is accessed (Maki \& Mermel, 1998). Needleless systems are now standard practice for use with CVADs. These include needleless syringes for accessing the PICC, but also new catheter hub endings and ports. Some studies have demonstrated an increased risk with these devices due to the device's design, or staff not adhering to maintenance procedures such as hand-washing, swabbing of the hub, and timely changing of the hub or port endings (Brown, Moss, \& Elliott, 1997; O'Grady et al., 2002a). Two studies revealed a decreased risk of infection when needle-less positive pressure connectors were used (Yebenes et al., 2003, 2004).

The longer the PICC is in place the more likely it is for infection to develop as this gives the microbes more time to grow (Raad et al., 1993). Hence the catheter should be removed as soon as it is no longer required in order to remove the infection risk altogether (Sanders, 2006).

\section{Thrombosis prevention}

Since there is a well established link between CRS and thrombosis, thrombosis prevention is an important aspect of care. Fibrin deposits attaching to the internal surface of the PICC lumen may promote the adherence of cocci and increase the risk of CRS. This phenomenon is covered later in this chapter.

\section{Other infection prevention measures}

Phlebitis (inflammation of a vein) is frequently associated with infection. Mechanical phlebitis can be caused by a large diameter PICC being inserted into a small vein 
resulting in restricted blood flow around the device and friction against the intima of the vessel (Mazzola, Schott-Baer, \& Addy, 1999). Unresolved mechanical phlebitis can develop into infective phlebitis presenting as a swollen, hot and painful PICC insertion site (Mahalm et al., 2003). This risk can be minimised by inserting the smallest PICC possible to meet the patient's treatment needs, and ultrasound guidance now allows for a larger vein (the basilic or brachial vein) to be accessed above the anti-cubital fossa at insertion.

Inserting antibiotic locks into CVADs has also been suggested and tested with promising results; however exposing patients to low levels of vancomycin could increase the risk of antibiotic resistance. A local study tested $70 \%$ ethanol locks in silicone Hickman catheters and found a statistically significant reduction in CRS rates (Sanders, 2006). Following Sanders' local Christchurch Hospital study, practice might have been adjusted so that suspected PICC infections were treated with $70 \%$ ethanol locks in order to prevent premature PICC removal, but unfortunately use of ethanol is not recommended by the manufacturer (Arrow ${ }^{\mathrm{TM}}$ ) with polyurethane catheters. This is likely to be because polyurethane catheters have thinner walls than silicone catheters (Renner, 1998) and ethanol may lead to degradation of the catheter. Literature shows that infection is the most common PICC complication, the second major complication of PICCs is venous thrombosis, which will be discussed next.

\section{Venous thrombosis}

After PICC placement, one of the major concerns is thrombosis. A thrombosis is where blood has changed from a liquid into a solid state, producing a blood clot (Hamilton, 2006). Any access device inserted into the vascular system increases the risk of thrombus formation, either in the blood vessel or in the catheter itself (BoweGeddes \& Nichols, 2005). Venous thrombosis is diagnosed by generalised swelling of the arm with radiological or ultrasound confirmation (Mahalm et al., 2003). Allen et al. (2000), in their retrospective study of PICC lines, reported symptomatic venous thrombosis rates based on clinical signs and symptoms to range from $1 \%$ to $4 \%$. However, they found the true incidence of thrombosis based on venography, to be much higher at $38 \%$. The highest incidence by site was cephalic vein $57 \%$, basilic vein $14 \%$ and brachial vein $10 \%$. Other researchers have also found that thrombosis 
occurs more frequently than is recognised and may, due to lack of signs and symptoms in the patient, be complicated by pulmonary embolism (Chemaly et al., 2002).

Thrombus formation in a vessel occurs for three primary reasons, known as the Triad of Virchow (Orr \& Ryder, 1993; Walshe et al., 2002). First, as a result of endothelial injury or irritation to the vein wall in which the PICC line is placed, secondly, from venous stasis, obstruction, or change in blood flow due to the catheter's presence, and thirdly, from platelet aggregation due to hypercoagulability, or a combination of these factors (Bowe-Geddes \& Nichols, 2005). Two catheter-associated variables are also related to thrombosis, namely catheter diameter and catheter tip location. Eastridge and Lefor (1995), in a retrospective study of 274 cancer patients with 322 CVADs inserted, identified that indwelling central catheters with a large diameter (12.5 French) tend to have a higher incidence of thrombosis and a shorter mean time until failure (40 days) than do catheters with relatively smaller diameter (10 French, with 146 days mean time until failure).

Although Eastridge and Lefor (1995) studied Hickman catheters as opposed to PICC lines, with all catheters inserted by a surgeon rather than specially-trained nurses, they found that $20 \%$ of all 12.5 -French triple lumen Hickman catheters needed removal because of venous thrombosis compared with only $7 \%$ of all 10 -French catheters. Eastridge and Lefor proposed that the reason for this disparity was most likely related to an increase in venous stasis and perhaps a greater degree of endothelial injury with the larger diameter catheters, as well as an increase in catheter manipulations because of more lumens. Grove and Pevec (2000) also found that catheter diameter was statistically significant as a risk factor for thrombosis. Another identified risk factor is high catheter tip placement within the superior vena cava resulting in a higher incidence of thrombosis than when the catheter tip is placed low in the superior vena cava. This is likely due to a higher degree of catheter tip mobility leading to a greater chance of endothelial injury and damage to the vessel wall (Kuter, 2004; Nightingale et al., 1997; Schwarz, Coit, \& Groeger, 2000).

Patient risk factors for CVAD-related thrombosis include previous history of deep vein thrombosis (DVT) and pulmonary embolism, a history of cerebro-vascular 
accident (CVA), hyper-coagulable state, or the presence of AIDS or malignancy (Chemaly et al., 2002). Malignancy seems to result in a higher rate of CVAD thrombosis than no malignancy (Kuter, 2004). Also, Anderson et al. (1989), in a very large study of Hickman catheters in oncology patients, found that some types of cancer may be associated with higher rates of catheter-related thrombosis. They found that $45 \%$ of patients with adenocarcinoma of the lung developed catheterrelated thrombosis compared with only $9 \%$ of those with head and neck cancer. Finally, the type of chemotherapy being infused may play a factor. At least one very small prospective study found that clotting occurred in six of $11(55 \%)$ catheters through which sclerosing chemotherapy was infused, but in only nine of 29 (31\%) infused with non-sclerosing chemotherapy (Bern et al.,1990).

PICC-related thrombosis refers to all types of thrombi, including fibrin sheaths, intraluminal clots, and deep vein thromboses.

\section{Fibrin sheath formation}

Within 24 hours of PICC insertion, a fibrin sheath forms around most catheters (Rosovsky \& Kuter, 2005). However, the presence of these sheaths does not predict DVT or embolisation of the vein in which the catheter is placed. Detailed studies have shown that these sheaths are always colonised by cocci (Kuter, 2004). There will be more discussion about fibrin sheaths later in this chapter when describing mechanical failure of PICCs.

Two forms of thrombi may develop in patients with PICCs, an intraluminal thrombus or a mural thrombus DVT. An intraluminal thrombus forms inside the catheter itself and can result in clotting within the lumen, causing partial or complete occlusion despite routine flushing with saline or heparinised saline (Bowe-Geddes \& Nichols, 2005). Anderson et al. (1989) found this to be a very common occurrence (93\%) in their study of Hickman catheters, however two other studies reported a frequency of 11-13\% (Ray, Stacey, Imrie, \& Filshie, 1996; Schwarz et al., 2000). Other causes of intraluminal occlusion are precipitation of incompatible drugs or lipid occlusions from parenteral nutrition (Hamilton, 2006). These lipid occlusions are more common with silicone PICCs, as lipid emulsion tends to adhere to silicone (Bowe-Geddes \& 
Nichols, 2005). Lipid occlusions require 70\% ethyl alcohol to dissolve.

Fortunately, most other intraluminal thrombi can be successfully lysed with fibrinolytic agents like urokinase or tissue plasminogen activator (Actilyse tPA), which are instilled for 30-120 minutes and then withdrawn from the PICC (BoweGeddes \& Nichols, 2005). If there is difficulty instilling the fibrinolytic agent, the 'POP technique' is utilised (Appendix 5). This is a mechanical percussive technique that has been researched and found in one study to restore patency in $86 \%$ of occluded catheters (Fetzer \& Manning, 2004). The technique uses a 10-ml luer-lock syringe containing one to two mls of normal saline attached to the hub of the occluded PICC. The syringe plunger is pulled back and released at two-second intervals until patency is restored. Releasing the plunger causes a 'pop' sound whilst sending a shock wave down the catheter.

Importantly, the inability to withdraw blood from the PICC does not necessarily mean an intraluminal thrombosis has formed. Gould, Carloss, and Skinner (1993) found that $57 \%$ of thrombosed CVCs versus $27 \%$ of non-thrombosed CVCs failed to draw blood when blood withdrawal was attempted. Another study using venography showed that $58 \%$ of catheters with blood withdrawal problems had thrombosis but $42 \%$ did not, demonstrating that an inability to withdraw blood from the catheter does not always mean it is occluded by thrombus. Non-thrombotic mechanical problems can commonly prevent blood flow hence diagnosis of the PICC problem is essential before removing the PICC (Stephens, Haire, \& Kotulak, 1995). These mechanical difficulties are described later in this chapter.

A mural thrombus in contrast, can form between the catheter and the vein wall of the PICC, and can also be partial or complete. Studies show that it affects $12-74 \%$ of all CVADs in general (Bowe-Geddes \& Nichols, 2005; Kuter, 2004). It is of interest to note that as many as $71 \%$ of patients with these mural thrombi are asymptomatic. In contrast, an average of $12 \%$ of all patients with CVADs develop a symptomatic thrombus describing symptoms such as arm, neck or head pain or swelling, erythema of the extremity, venous distension, or numbness of the extremity. Thus only about one in three mural thrombi result in symptoms (Kuter, 2004). In a small number of longitudinal studies reviewed by Kuter, most CVC-related thrombi occurred within 
30 days of insertion, with $15-30 \%$ of cases causing postphlebitic syndrome and $11 \%$ causing pulmonary embolism.

In addition, thrombosis is a major risk factor for infection (Rosovsky \& Kuter, 2005). According to Hamilton (2006), the potential for micro-organisms to reside within thrombi is well known. This is not perhaps surprising since, as previously stated, almost all cannulated vessels contain a fibrin sheath that is seeded with adherent cocci (Kuter, 2004). The presence of a blood clot may provide nutrients for microbes to proliferate, leading to infection. Therefore, regular flushing of the PICC is essential to reduce the risk of thrombus formation (Hamilton, 2006).

To minimise the risk of thrombus formation, the smallest PICC possible to meet the patient's needs should be inserted, and a post-insertion X-ray should be checked to ensure the PICC catheter tip resides in the lower third of the superior vena cava (SVC) where a turbulent circulation will reduce the risk of thrombosis.

Efforts have been made to reduce CVAD thrombosis with prophylactic anticoagulation using low-molecular-weight heparin (LMWH). Monreal et al. (1996), in a prospective study of oncology patients with Portacaths, found a statistically significant difference in outcome $(p=0.002)$ for those receiving dalteparin vs. no therapy: DVT developed in $1 / 16$ patients $(6 \%)$ taking dalteparin vs. $8 / 13(62 \%)$ in those receiving no anticoagulant therapy. However, similar more recent studies using larger patient numbers, have failed to show any statistical difference (Kuter, 2004; Reichardt et al., 2002). Hence, lack of clear and unequivocal evidence has halted this practice in prophylactic use of LMWH to prevent thrombosis in oncology patients with CVADs, in many centres internationally.

Low-dose warfarin prophylaxis (usually $1 \mathrm{mg}$ /day) to prevent thrombosis has also been trialled in oncology patients with CVADs. Although several older trials supported its use (Bern et al., 1990; Boraks et al., 1998), two more recent studies in oncology patients (Eastman et al., 2001; Heaton, Han, \& Inder, 2002) showed no benefit. Although these studies may reflect better catheter care and design, the routine use of low dose warfarin cannot be currently justified in this patient group, especially in those on fluorouracil (5-FU) chemotherapy, or who have impaired liver 
function or inadequate nutrition (Magagnoli et al., 2003; Masci et al., 2003;

Rosovsky \& Kuter, 2005). Not only was venous thrombosis a potential concern, but factors surrounding failure of the catheter itself also emerged.

\section{Mechanical failure}

Mechanical failure includes catheter malposition (and PICC migration), catheter occlusion caused by mechanical obstruction, and catheter damage, such as rupture or breakage. Some researchers include catheter occlusion under this heading while others place it alongside thrombosis complications. In this study it has been placed under both headings. Previously described were catheter occlusions caused by thrombosis, however catheter occlusion can also occur due to mechanical obstruction, which can be internal or external (Farjo, 2003). Internal mechanical obstruction of the PICC can result from the catheter tip migrating into a smaller vein or the tip resting against a vessel wall. Even when the catheter tip is in the superior vena cava, it can become obstructed due to 'Pinch-off' syndrome, a condition where the catheter can become compressed between the clavicle and the first rib. External mechanical obstruction occurs when kinked PICCs or clamped tubing compress the PICC lumen.

Non-thrombotic occlusions can be caused by extraluminal events such as a fibrin tail or fibrin sheath, causing partial or complete occlusion (Farjo, 2003). A fibrin tail occurs when fibrin, blood cells and platelets accumulate and adhere to the tip of the catheter, occluding the back flow of blood and creating a one-way valve. Intravenous fluids can still be infused but blood sampling can become difficult. When the fibrin tail totally encases the PICC tip, both infusion of fluid and blood withdrawal become impossible (Bowe-Geddes \& Nichols, 2005). Other causes for extraluminal occlusion include previous radiotherapy to the axilla or supraclavicular fossa, or enlarged and/or removed large axillary nodes. This is one of the reasons why PICCs are placed on the side furthest from surgery of women with breast cancer.

Stephens et al. (1995) point out not to assume that catheter access failures, for example, not being able to withdraw blood, are caused by thrombus formation. They found that $40 \%$ of the catheter dysfunctions in their study were caused by non- 
thrombotic causes such as catheter tip displacement, which were salvageable once proper diagnosis, was made.

Catheter malposition can occur due to catheter migration or intrathoracic pressure usually caused by coughing or vomiting. Since vomiting and/or coughing can be very common symptoms in oncology patients, it is important for oncology personnel to be mindful of this mechanical complication. If malposition is suspected the distal tip termination should be confirmed by chest $\mathrm{x}$-ray because malposition can lead to serious complications. For example, PICC positioning proximal to the superior vena cava can lead to phlebitis and thrombosis formation or, if the PICC tip moves into the distal right atrium or right ventricle, dangerous cardiac arrhythmias may occur (Wise, Richardson, \& Lum, 2001). Ensuring the catheter is adequately secured is essential to preventing catheter dislodgement or migration. Studies have shown that the use of stat-lock securement devices is safer and more effective than suturing the PICC in place (Bowe-Geddes, \& Nichols, 2005). Further to potential mechanical failure, it appears that different types of catheters have differing complications.

Catheter damage can occur with any PICC. This can be caused by defective products but is more often precipitated by improper care and maintenance. Sometimes excessive pressure is inadvertently applied when flushing the PICC. Use of syringes smaller than $10 \mathrm{cc}$ can cause excessive intraluminal pressure which may result in catheter rupture. Counter to this, use of syringes larger than $5 \mathrm{cc}$ when withdrawing blood from the catheter can create a vacuum, resulting in the catheter collapsing. Other documented causes of damage include contact with sharp objects such as scissors, entangling the external portion of the PICC in equipment, bed linen or patient clothing, or applying luer locking devices too tightly and cracking the catheter hub (Bowe-Geddes \& Nichols, 2005).

Once a catheter has been damaged, it is contaminated and will need replacing or repair. The literature seems to suggest that PICC line repair is required more often with silicone PICCs than polyurethane PICCs. One study showed that polyurethane catheters had superior flow rates and tensile strength when compared to silicone catheters (Angle et al., 1997). Silicone catheters have thicker walls and this causes slower flow rates thus increasing the risk of thrombus formation. Catheter rupture or 
fracture most often affects silicone PICCs, according to Masoorli (1997). A study by Renner (1998) also described silicone catheters experiencing snapping or fracturing more easily than polyurethane catheters. The reason proposed was that they were less smooth, flexible and less hydrophilic than polyurethane.

The silicone (Groshong ${ }^{\mathrm{TM}}$ ) catheter (unlike the polyurethane catheter used at Christchurch Hospital) has a unique three-way slit valve, which theoretically requires less care than other non-valved catheters because the valve is designed to prevent blood reflux into the catheter tubing. This allows for the PICC to only be flushed weekly when not in use. However, one study found that blood was visible in the translucent Groshongs even when the catheters had not recently been used (Mayo et al., 1995). In Mayo et al.'s study, adherent blood clots were found in 27 out of 28 removed Groshong catheters, leading them to conclude that the valves of the Groshong catheter commonly allowed blood to reflux inward and that in most cases this blood was concentrated enough to clot. This finding would indicate that silicone (Groshong ${ }^{\mathrm{TM}}$ ) PICCs are more likely to occlude than polyurethane (Arrow ${ }^{\mathrm{TM}}$ ) PICCs which are flushed daily when not in use. Furthermore, Pasquale et al. (1992) reported a statistically significant increase in catheter malfunction with silicone (Groshong ${ }^{\mathrm{TM}}$ ) PICCs compared with Hickman catheters.

The Christchurch Hospital PICC insertion service chooses polyurethane catheters over silicone catheters because of the literature describing problems with silicone PICCs but also because they are deemed more pliable and able to be inserted with greater ease. Although they require daily flushing rather than weekly flushes, it is considered a reasonable compromise given the potential for fewer catheter problems.

\section{PICC costs and complication rates}

Research has established that PICCs are significantly less expensive to insert than tunnelled CVCs or implanted vascular ports because they do not require a general anaesthetic or a surgical procedure for insertion (Cheong et al., 2004). Horattas et al. (2001) retrospectively reviewed all CVADs inserted at their hospital over a 30-month period to compare indications, costs and complications. These included 126 surgically placed CVCs, 264 PICCs, and 294 radiologically-inserted ports. They 
reported a cost saving of US\$3000 - \$4000 per patient for PICC insertion compared with Portacaths and other surgically placed central catheters. In their study PICCs were primarily inserted by nurses with radiological back-up only for difficult insertions. Having specially-trained nurses inserting PICCs, rather than radiologists, significantly reduces costs (Masoorli \& Angeles, 1990; Schmid, 1994, 2000).

Horattas et al. (2001) also describe the cost benefits of PICCs in regard to complications when compared to other CVADs. According to Ryder (1995), centrally placed catheters increase the risk of serious complications including pneumothorax, tension pneumothorax, haemothorax, hydrothorax, hydromediastinum and tracheal puncture. However, with PICCs, these particular risks are virtually non-existent (Horattas et al., 2001; Ryder). The additional costs from a haemothorax alone complicating a central line, is reportedly US $\$ 8300$ (Ryder). Such significant complication costs are not incurred with PICC lines.

PICC cost effectiveness compared to other CVADs has also been described by other researchers (Cardella et al., 1996; Ng et al., 1997; Smith et al., 1998; Walshe, et al., 2002). They are also easier to insert and remove than other CVADs (Snelling, Jones, Figueredo, \& Major, 2001). There is growing evidence to suggest that there are fewer complications with PICC lines than central lines (Goodwin \& Carlson, 1993; Mahalm et al., 2003; Ng et al.; Schmid, 2000; Snelling et al.). Ng et al. outline three separate studies that found that PICCs have a lower incidence of infection compared with subclavian, internal jugular and femoral percutaneous catheters.

\section{PICC studies specific to oncology patients}

Few published studies reported on PICC complications specifically amongst oncology patients. Four studies reflected the clinical situation similar to this present study and were pivotal in informing the present study's methodology and choice of design. First, Walshe et al. (2002) followed all oncology patients who had a PICC successfully inserted over a 12 month period. Since this study was the largest prospective study defining the outcome of PICCs placed in patients with cancer; it was deemed appropriate that it should be considered in the planning of this present study. Walshe et al. adopted a prospective observational study. Even though it 
explored silicone (Groshong ${ }^{\mathrm{TM}}$ ) catheters, the cohort had similarities to this study. It included 351 paediatric and adult oncology patients, the majority (282) of whom had solid tumours. There were 42 patients with haematological cancers, five with bone marrow transplantation, and 22 patients with no cancer. Insertions were done by both nurses at the bedside and radiologists in the radiology department. Walshe et al. found that $115(32.8 \%)$ of the 351 PICCs required premature removal due to a complication, for a rate of 10.9 per 1000 catheter days. It is important to note that patients with haematological malignancy $(\mathrm{p}=0.03)$ and bone marrow transplant $(\mathrm{p}=$ 0.05) were at an increased risk for CRS and those with metastatic disease were at a reduced risk $(p=0.03)$. It was found that infection was the most common complication, followed by phlebitis (23/351) and leaking or broken catheter (21/351). This was followed by accidental removal (19/351), PICC occlusion $(14 / 351)$ and vein thrombosis (12/351).

Secondly, aspects of interest in Cheong et al.'s (2004) PICC study were that it was a retrospective study of 27 patients with solid tumours; conducted in an Australasian context and demonstrating a high complication rate of $40.7 \%(11 / 27)$. Infection complications were high at $25.7 \%$ (7/27) for a rate of 8 per 1000 PICC days. They claim that they found a high complication rate, which indeed they did, however it was a very small oncology cohort. It seems that they were concerned enough with the high complication rate, and arguably the smallness of the study, to implement strategies to reduce complications. These included staff and patient education, insertion technique modification and PICC maintenance utilising a PICC nurse. The PICC nurse's role was to educate nurses on PICC aftercare, ensure adherence to the formal PICC aftercare policies, maintain an audit of the PICC line complications as well as unblock and repair PICC lines. They also adopted the intervention of $2 \%$ chlorhexidine for insertion and PICC dressings. The insertion practice was modified to use larger drapes and a strict cap and mask policy. All of the silicone (Groshong ${ }^{\mathrm{TM}}$ ) PICCs were inserted by a radiologist.

Thirdly, at the same Australasian centre as Cheong et al. (2004), Yap et al. (2006) improved the investigation procedures by utilising a prospective design. They evaluated the impact of the interventions that had been instigated to improve the PICC complication rate and were then keen to compare the findings to their previous 
study (Cheong et al., 2004). Significant improvements in their design are noteworthy. They studied prospectively, still utilising medical records, with a larger cohort of 88 PICCs in 73 patients. It emerged that the complication rate had dropped to $15.9 \%$ $(14 / 88)$ or two complications per 1000 PICC days. They reasoned that the strategies introduced were instrumental in this reduction.

Fourthly, only one unpublished study was found to have researched the efficacy of polyurethane (Arrow ${ }^{\mathrm{TM}}$ ) catheters (Mahalm et al., 2003). In Mahalm et al.'s local, Christchurch Hospital-based prospective unpublished study of 565 polyurethane PICCs and 127 Midline insertions in general hospital patients over a 20 month period, $23.6 \%$ of PICCs were prematurely removed due to blockage (25), catheter migration (8), accidental removal (7), catheter failure (55), and suspected or confirmed infection (61). The high incidence of catheter failure was related to use of $\left(\right.$ Biovue $^{\mathrm{TM}}$ ) polyurethane catheters which were withdrawn from the Australasian market towards the end of the study period because of unacceptable problems with catheter failure. Polyurethane (Arrow ${ }^{\mathrm{TM}}$ ) PICCs were used thereafter with significant anecdotal improvement however no further research had been undertaken until the current study to establish the rate of complications with these.

\section{Other oncology studies}

Other published studies showed similarly high rates of complications in cancer patients. Last et al. (1998) compared complications of PICCs and Hickman catheters in 130 solid tumour patients and reported superiority of PICCs over Hickman catheters. However, the overall PICC complication rate of $38.5 \%$ was high with a serious complication rate of $25 \%$. Smith et al. (1998), in a retrospective review of 838 surgical patients, found a significantly higher complication rate of $44 \%$ in PICCs inserted for chemotherapy.

Another prospective study (Snelling et al., 2001) compared survival time of tunnelled CVADS with PICC lines in patients receiving infusional fluorouracil (5-FU) chemotherapy. They found that survival time was similar for both devices for the first 120 days, but after that the tunnelled CVAD survival was statistically better than PICCs $(p=0.051)$. They concluded that PICCs were less invasive, more cost 
effective and easier to schedule for 5-FU chemotherapy, however their advantage over tunnelled CVADs decreased significantly after 120 days.

\section{Summary}

In summary then, most studies of complication rates following PICC insertions are in non-oncology patients. There are few studies exploring not only PICC lines generally, but more specifically their use in oncology patients. Overall, the literature revealed three main complications; infection, thrombus formation, and mechanical failure. Infection rates were higher in oncology patients especially those with neutropenia and poor nutritional status, and more prevalent amongst CVADs with multiple lumens, higher number of catheter manipulations, thrombosis and poor insertion and PICC maintenance techniques.

Thrombus formation was found to be related to catheter diameter and high catheter tip placement in the SVC. Patient risk factors included previous history of DVT or CVA, hyper-coagulable state, or the presence of AIDS or malignancy, particularly adenocarcinoma of the lung.

Mechanical failure was attributed to catheter tip displacement, catheter migration or malposition, excessive pressure when flushing the PICC, inadequate construction, fibrin sheath formation, lipid occlusion, kinking of the catheter, and whether the catheter was silicone or polyurethane.

\section{Statement of research problem}

A review of relevant literature surrounding the use of PICCs has identified that there are a limited number of studies on PICC lines in oncology patients, most report on silicone (Groshong ${ }^{\mathrm{TM}}$ ) catheters, and results in these studies vary. Hence it was deemed to be of value that PICC line complications in polyurethane (Arrow ${ }^{\mathrm{TM}}$ ) PICCs and their rate of occurrence be accurately identified in an oncology cohort. Such a study had therefore the potential to obtain results which may lead to changes in nursing practice for PICC line management, as well as validating whether the most efficient device was in use for PICC lines within an oncology context. It would also add to the literature on this important area of nursing practice and patient care. Given 
that patient satisfaction is very high for use of these devices (Chernecky, 2001;

Mahalm et al., 2003; Walshe et al., 2002); it is important to formally identify possible complications. This identification is central to the proposed study. The next chapter, Chapter 3, describes how PICCs are clinically inserted and managed. 


\section{Chapter Three: Clinical aspects of PICCs}

This study examines peripherally inserted central catheter (PICC) complications and rates within a cohort at Christchurch Hospital's oncology service. This clinical chapter provides an in-depth explanation of the PICC (Arrow ${ }^{\mathrm{TM}}$ ) used at Christchurch Hospital, how patients are selected for PICC insertion, and the PICC insertion process within the facility. Explanation is also given as to how PICCs are managed post-insertion and the education process surrounding this for both staff and patients.

\section{Established benefits of PICCs}

PICCs have been associated with lower infection rates compared with other nontunnelled central venous catheters (CVCs) (Mahalm et al., 2003; Ng et al., 1997). They are significantly less expensive to insert than tunnelled CVCs or implanted vascular ports (Cardella et al., 1996), and there is no risk of pneumothorax at PICC insertion (Bowe-Geddes \& Nichols, 2005). Furthermore, they are easier to insert and remove than other central lines, and are cost-effective (Cardella et al.). Part of the justification for PICCs being cost effective is that they can be inserted by registered nurses who have been specially trained in this procedure. In contrast other tunnelled CVCs require surgeons and operating theatre time for insertions (Bowe-Geddes \& Nichols, 2005; Orr \& Ryder, 1993). There is also evidence to suggest that there are fewer complications with PICCs than other central lines (Goodwin \& Carlson, 1993; Mahalm et al., 2003; Ng et al., 1997; Schmid, 2000). These and other research findings were discussed in greater detail in the last chapter.

\section{The nature of PICCs}

The polyurethane (Arrow ${ }^{\mathrm{TM}}$ ) PICC lines used at Christchurch Hospital are imported from the United States of America and are produced by Arrow International Inc. manufacturers. They are all $55 \mathrm{cms}$ long, and upon insertion require $\mathrm{x}$-ray confirmation that the tip is correctly positioned in the lower third of the superior vena cava (SVC) prior to use. Furthermore, because PICCs are peripherally placed, and are neither tunnelled nor implanted, surgical intervention is not required (Walshe et al., 2002). Compared with other peripheral catheters, they can usually remain in 
place longer, thus decreasing venous irritation from drug therapies and minimising the need for venous punctures when prolonged treatment administration is required.

\section{PICC use for treatment}

PICCs are often used for patients receiving vesicant or irritant chemotherapies, longterm or large chemotherapy regimens, long-term treatment with intravenous (IV) antibiotics, total parenteral nutrition (TPN), or repeated administration of blood or blood products. They can also be used for infusion of irritant medications or solutions, or infusions of hyper/hypotonic solutions or those with extremes of $\mathrm{pH}$. This is because the catheter tip resides in the lower third of the SVC and blood flow around the catheter is high (usually 2 litres or more per minute). Thus immediate dilution of the infusate helps to prevent chemical irritation of the vessel walls by the prescribed therapy (Bowe-Geddes \& Nichols, 2005). Furthermore, PICCs are ideal for patients with limited venous access or who have experienced trauma or surgery to the chest or peripheries. For patients who require long-term IV therapy, such as antibiotics for osteomyelitis, PICCs allow such treatments to occur as an outpatient rather than in hospital. Patients who are having infusional chemotherapies, (that is, chemotherapies that are given slowly intravenously over 24 hours to one week, or longer), require a PICC in order for treatment to be given as an outpatient rather than in hospital. PICCs also allow ready access for blood sampling and are helpful for patients with needle phobias who require frequent or long-term treatment. PICCs also allow for early hospital discharge with ongoing treatment at home or as an outpatient.

\section{PICC insertion and management}

At Christchurch Hospital PICCs are generally inserted by a team of specially trained nurses in the Digital Subtraction Angiography (DSA) unit, the interventional suite within the Radiology Department. Insertions are performed under ultrasound and fluoroscopy guidance at an average cost of NZ\$200 per insertion. To ensure cost efficiency, to be considered eligible for a PICC line patients are expected to need IV therapy for at least seven days. Seven days in situ is the cut off to achieve cost neutral status. Once inserted, an average of about 50 people have their PICC treatment managed in the community at any one time (Doryan Mahalm, Charge Nurse Manager, Radiology, personal communication, 19 November, 2006). 
Previously, PICCs at Christchurch Hospital were inserted into the antecubital fossa of the right arm, as this was the easiest insertion point. Since 2005, ultrasound guidance has become routinely available in the DSA unit, allowing the PICC to be inserted into a person's non-dominant arm and above the antecubital fossa into either the basilic or brachial vein of the upper arm (Mahalm et al., 2003). This change has appeared to make the device easier to hide and less vulnerable to phlebitis and damage by overuse of the arm. Since normal use of the arm is important to minimise the risk of thrombus formation, this change in entry point is seen to be advantageous in terms of risk and complication minimisation. Anecdotally, this positioning is thought to have significantly reduced the risk of mechanical phlebitis. Furthermore, patients have described increased satisfaction with PICC placement since ultrasound guidance has allowed the PICC insertion to be above the elbow rather than at the elbow (Polak, Anderson, Hagspiel, \& Mungovan, 1998).

Oncologists at Christchurch Hospital make the decision whether their patients would benefit from PICC placement for treatment options. A history is taken at the time of decision-making to ensure the line is well suited to the patient. Most daily activities are manageable with a PICC but some activities that require repetitive or strenuous movements of the PICC arm can increase venous access problems and thus need to be limited; for example, keen golfers and swimmers may not be suited to PICC insertion due to repetitive use of their arms. Bowe-Geddes and Nichols (2005) recommend that the oncologist needs to select the most appropriate central venous access device for the course of therapy, being mindful of minimising pain and venous damage, utilising nursing time efficiently, being cost effective and considering the patient's lifestyle needs. Also, PICC insertion can become difficult or even impossible for patients who have had multiple previous PICCs. Therefore, if a patient requires frequent but intermittent access, an implanted venous port (Portacath) may be a more appropriate choice.

Another important consideration in whether a patient has a PICC line inserted is their ability to adhere to the restrictions that a PICC line imposes. An assessment of their ability to undertake the daily maintenance tasks such as flushing the line and keeping the site and extension tubing dry needs to be made. The patient also needs to be available for the weekly redressing of the insertion site by a nurse. Because the PICC 
is a central line, infection and other complications are possible, thus careful PICC management by the patient is paramount. The extension set, clamp and CLC 2000 ending, as well as the point of entry to the skin are best kept dry, which means covering it with plastic film and wearing a waterproof sleeve when showering. Swimming or other activities that risk getting the PICC wet should be avoided. If the patient is not in a position to attend to these essential management activities, then other means of treatment administration may need to be considered, most likely a peripheral intravenous (IV) line. In practice, most patients and their families manage the PICC well.

Once the oncologist has recommended PICC insertion, the procedure is booked in at the digital subtraction angiography (DSA) suite and the patient is ideally sent for a one-on-one nursing education session in the Oncology Department to discuss the PICC insertion, management and chemotherapy regimen. The patient is given an information booklet compiled by the Canterbury District Health Board (CDHB) called A Guide to your PICC/Midline: CLC2000-Saline Lock (Appendix 2) (reproduced with permission of CDHB, see Appendix 9). This takes the patient step by step through the nature of a PICC, its insertion and management, and guidance on when to seek professional help. Furthermore, the guide explains self-administration of saline flushes. The patient is also given an Agreement to Treatment form which requires explanation to the patient prior to their signing it, thus gaining informed consent. This is normally signed by the patient just before insertion.

Prior to insertion the patient is encouraged to be well hydrated and if they are unable to achieve this, the giving of $500 \mathrm{ml}-1000 \mathrm{ml}$ of IV fluids is recommended, as dehydration can increase venous problems, making it difficult to cannulate the patient. It is thought that if the patient is well hydrated, there can be a reduction in insertion trauma and phlebitis. Ideally, the patient's arm is kept warm for insertion. This stimulates blood flow and return via the large peripheral veins, making cannulation easier.

In the DSA suite, the PICC-insertion nurse discusses the procedure with the patient as part of informed consent. Explanation is given of limitations on activities, potential complications and their management, ongoing monitoring and management of the PICC and site care, plus care of emergent situations. The potential 
complications and how they are minimised are discussed. These include, bleeding from the site of the insertion, infection, thrombus formation, and the importance of normal movement of the arm. Also discussed are tip malposition, embolus and mechanical phlebitis. When the nurse is confident the patient understands the written and verbal information, the patient is requested to sign the Agreement to Treatment (consent) form. These potential complications were discussed in detail in the previous chapter.

The nurse identifies the arm for insertion. Ideally, this is the non-dominant arm or in women with breast cancer, the arm furthest away from the affected breast. The patient is then positioned with the insertion arm placed at a 45 degree angle to the body. The appropriate blood vessel for insertion is located using a tourniquet and ultrasound guidance. The presumed anatomical course of the chosen vessel is measured. The basilic or brachial veins are the most commonly used veins for insertion because they are the largest and have the straightest pathway in the upper arm. The brachial vein is usually undamaged even in patients with a history of many IVs, but needs ultrasound guidance because of its deep location.

In preparation for insertion, the health professional performing the insertion should pre-wash their hands with a chlorhexidine-based soap, and adopt 'Full Barrier Draping' to the area as well as skin preparation with $2 \%$ chlorhexidine/alcohol solution. This sequence in the procedure is recommended in international guidelines by O'Grady et al. (2002a) and followed as usual practice surrounding PICC insertion at Christchurch Hospital. Following this, a tourniquet is applied and tightened and the appropriate vessel is located using an ultrasound probe. Local anaesthetic (lignocaine 1\%) is injected followed by a needle and introducer, and the PICC is threaded over the wire introducer before the introducer is removed. This process is called the modified Seldinger Technique (Bowe-Geddes \& Nichols, 2005). Fluoroscopy (video x-ray) is then used to follow the passage of the catheter and to confirm that the catheter tip is in the lower third of the superior vena cava.

The external length of the catheter is measured and recorded and the PICC is then well anchored down with steristrips and a stat-lock device (which further secures the external length of the catheter to the arm). The entry point of the catheter is then covered with gauze to capture any ooze that might escape. Once inserted the nurse 
completes insertion documentation including catheter information, size, insertion procedure, site of insertion, difficulties with insertion and tolerance to procedure, tip confirmation and blood return as well as written and verbal instructions (Appendix 3).

The PICC is normally bandaged firmly for the first 24 hours after insertion to prevent bleeding from the insertion site. It is recommended that heat be applied over the cannulated vein for up to 24 hours post-insertion, particularly if the insertion has been traumatic. The heat swells the cannulated vein improving blood flow and moving the vein wall off the catheter, reducing the incidence of mechanical phlebitis (IVNNZ PICC study day handout). After 24 hours the dressing is changed, the site cleansed and a transparent semi-permeable dressing is applied. This acts as a bacterial barrier over the access site resulting in a drier catheter site, thus reducing skin maceration and lowering skin colonisation, which in turn reduces the risk of catheter-related infections. Following this, the dressing is changed weekly unless the insertion site bleeds, or the dressing gets soiled or wet in the shower. The site needs to remain dry and occlusive at all times in order to protect the sterility of the catheter and maintain the catheter position.

The PICC dressing is changed every seven days by the oncology nurse or district nurse. The protocol for dressing change and accessing the PICC is clearly described in Appendix 4 (reproduced with permission of CDHB, see Appendix 9). At Christchurch Hospital oncology patients have a positive displacement valve, called a CLC2000 (ICU Medical, San Clemente, California), attached proximally to the catheter hub. This device is designed to prevent the reflux of blood into the catheter, thus allowing the PICC to be flushed with normal saline only and not requiring a heparinsed/saline lock (Bowe-Geddes \& Nichols, 2005). The PICC dressing protocol involves the nurse checking the insertion site carefully, checking the catheter external length, cleaning the site with moderate friction using a chlorhexidine gluconate $2 \%$ swab, changing the CLC 2000 ending once it has been primed and applying new steristrips, statlock and IV $3000^{\mathrm{TM}}$ transparent dressing aseptically whilst wearing sterile powderless gloves. Following a local Christchurch Hospital study by Chambers et al. (2005) (Chapter 2), standard practice is now to incorporate a Biopatch into the PICC line dressing regimen of neutropenic or other at-risk cancer 
patients.

The PICC is now ready to be accessed. This may include the giving of chemotherapy, the taking of blood, and the giving of IV therapy or red blood cells. It is recommended that concurrent to use of the PICC, patient education on flushing of the line and safe management of the PICC is undertaken at each patient-nurse contact. The nurses in the Oncology Outpatients' Department or the oncology ward are responsible for accessing the PICC lines for blood specimens or the giving of chemotherapy or other IV therapies such as antibiotics or red blood cells. These nurses had undergone a one-day training workshop to prepare them for this task. They are experienced nurses who have passed IV certification and are proficient at administering IV medication. The registered nurses are directed to follow predetermined guidelines/protocols for nursing access of the PICC (Appendix 4). Those giving chemotherapy have also undertaken and passed a special Cytotoxic Training Course. They were also the ones who were responsible for PICC education on safe flushing and management of the lines by the patient or their support person at home. District nurses, who underwent similar training within their facility, also had access to the PICC line in the patient's own home to undertake the weekly aseptic changing of the PICC site dressing.

\section{Patient education}

Patient education covers two main areas: firstly, how to flush the PICC line and secondly, an understanding of potential complications so that if one were to arise the patient would know what to do. Both of these patient education subject areas are now described in more detail.

\section{Flushing the line}

The patient and their support person are taught how to administer a saline pulsatile flush, as only the nurse administers the medication. This education can be commenced prior to discharge and continued by the district nurse in the community or the Oncology Outpatients' Department nurse. Education includes effective hand washing, how to prepare the site and how to use a non-touch technique to administer the saline flush. Once the syringe is inserted into the CLC2000 the patient is taught 
to firmly depress the plunger on the syringe using a 'push, pause, push, pause' technique.

The pulsatile saline flush creates turbulence within the catheter to help keep the catheter clear of any residual medication, blood or fibrin deposits within the catheter lumen. It is helpful for the patient and their support person to have performed this task at least three times successfully under the supervision of the nurse, before independently undertaking this at home. To keep the catheter ready for use, a saline flush is inserted into the CLC2000 daily. Ten millilitres of sodium chloride injection BP $0.9 \%$ (normal saline) in a luer lock syringe is used to flush the catheter hub to hub. All flushing should be done with $10 \mathrm{cc}$ or larger syringes, to prevent excessive flushing force damaging the PICC (Macklin \& Chernecky, 2004). Although the majority of patients can manage to flush their lines, if the patient or their support person is unable to flush the line safely, the district nurse or hospital nurse will flush the line daily for them. Mostly it would be the district nurse who would go into the patient's home every day and do this for them.

\section{Understanding potential complications}

Education begins whilst the patient is in hospital and once discharged the district nurse supports the patient in the care of the catheter and continues the education process. It is pointed out when to seek help with the catheter, for example, if the patient feels unwell, if they have a temperature above 38 degrees Celsius or develop chills or sweats. All patients are advised that if these symptoms occur they are to contact the Oncology Department at Christchurch Hospital. After hours calls are redirected to the oncology ward. They are also advised to seek advice if the dressing becomes loose, moist or falls off, or if they see any swelling, redness, blood, pus or other liquid under and around the dressing.

The patient is also advised to seek help if resistance is felt when flushing the line. This may indicate catheter blockage, which may need nursing intervention. If medication is being administered via the PICC line the patient needs to report if any fluid is seen leaking from the catheter or the extension set. If this were to occur the patient is educated to check that the connections are tight, to clamp the catheter, to cover the area of leakage with sterile gauze, and then tape or bandage the site and to 
then contact the nurse. If the catheter is accidentally removed the patient and their support person are advised to cover the insertion site with sterile gauze and a bandage, and to press firmly over the site for at least three to five minutes to attempt to stop the bleeding and to contact the nurse for further assistance.

If the catheter is split or cut, bleeding may occur or air may enter the catheter. The patient and their support person are taught how to kink or clamp above the break to stop bleeding. The catheter may need to be held in order to stop movement in or out of the arm. In this case it is advisable to contact the nurse or ambulance immediately. If air has entered the catheter, it is important that the patient lie flat and turn onto their left side, remembering to kink or clamp the catheter.

Very rarely, when receiving drug therapy, a reaction may occur. The patient and their support person are advised to seek medical help immediately if a rash develops, there is itchiness or swelling at the site or they suddenly become unwell. For example, if they develop a wheeze, shortness of breath or experience chest pain, the process would then be to ring for an ambulance.

Providing complications do not occur, PICCs can remain in place for one week to one year, or even longer. If however a complication does arise, part of managing this is to determine whether the PICC line should remain in situ.

\section{Minor complications}

Minor complications can be defined as complications that can usually be corrected with minor treatments and which do not necessitate the removal of the PICC. These include skin reactions to the dressing covering the insertion site, phlebitis of the catheterised vein, pain or bruising at the site, and sluggish blood withdrawal or resistance when flushing the PICC if they are able to be remedied by instillation of urokinase.

Skin reactions to the transparent semi-permeable dressing (usually IV3000 dressing) are not uncommon but usually respond well to treatment. The dressing is changed to another type of transparent semi-permeable dressing as soon as possible, and the skin reaction normally settles after a few days. If it persists, gauze is the 
dressing of choice. Rarely would the skin reaction be so severe to warrant withdrawal of the PICC.

Phlebitis is inflammation of the intima of a vein and is characterised by pain and redness along the course of the vein, with erythema and swelling at the site. This can occur within the first 24 hours of insertion and usually responds well to heat compresses. Mechanical phlebitis can be more severe and was discussed in more detail in Chapter 2.

Bruising at the insertion site is usually caused by a difficult insertion or bleeding after insertion. This normally settles within a few days with minimal intervention. Pain at the insertion site is usually related to phlebitis or difficult insertion, and usually settles with heat compresses.

Resistance when flushing or sluggish blood withdrawal may be due to fibrin sheath formation around the catheter or a fibrin tail at the distal end of the catheter. This resistance can usually be unblocked by the 'POP' technique (Appendix 5) or by instillation of urokinase.

Because minor PICC line complications can usually be corrected with conservative management or minor treatments, and do not normally lead to early PICC line removal, they are not the subject of this research study and not reported on in the findings.

\section{Major complications}

Major complications can be defined as more serious PICC complications that mostly result in early removal of the PICC line. The three major PICC complications as discussed in depth in Chapter 2 are infection/sepsis, thrombosis, and mechanical failure. Each of these is dealt with differently.

If infection or sepsis is suspected, this is potentially very serious for the patient, especially if they are immuno-compromised or neutropenic as a result of chemotherapy. Confirmation of infection is made by comparing PICC blood cultures and peripheral blood cultures taken when the patient has a fever $>38.5$ degrees Celsius. If the PICC blood cultures are positive and the peripheral vein cultures are 
negative, this provides reliable diagnosis of catheter-related infection (Elliot, Faroqui, Armstrong, \& Hanson, 1994). The decision whether to remove an infected PICC normally depends on the patient's condition, and the type and cause of infection. If not removed, the patient is normally hospitalised and administered IV antibiotics such as Vancomycin, with or without Gentamycin depending on the degree of sepsis. If the patient's condition does not improve, the PICC will be aseptically pulled and the catheter tip sent to the lab for analysis.

The second type of infection is skin-tunnel infection which occurs when organisms enter via the insertion site. It is often caused by staphylococcal infection where the patient's own skin flora enters the body at the point where the catheter exits and enters the body. Symptoms usually include pain, swelling and erythema at the insertion site. This may require systemic antibiotics, and occasional PICC removal.

Thrombosis, on the other hand, is treated with subcutaneous clexane 24-hourly in the first instance, followed by low dose warfarin therapy (usually $1 \mathrm{mg} /$ day). The PICC line itself may or may not need removal depending on the degree of symptoms, but in most instances the PICC is removed.

Mechanical failure of the PICC includes occlusion of the catheter which can be caused by intraluminal or extraluminal clots. Intraluminal occlusion is caused by clotted blood in the PICC, precipitation of incompatible drugs, or lipid build-up from total parenteral nutrition. If the device is totally occluded, removal or replacement of the PICC may be the only option. Withdrawing blood from a PICC is a known risk of causing PICC occlusion, unless flushed very well with two 10cc syringes of normal saline using the pulsated, push-pause method, creating turbulence in the catheter lumen (Todd \& Hammond, 2004). Extraluminal occlusion is usually diagnosed when it is possible to infuse fluids but impossible to aspirate blood. This is sometimes called persistent withdrawal occlusion (PWO). Causes can include fibrin sheath formation at the distal end of the catheter, malposition of the catheter tip, or anatomical obstruction. Urokinase is usually instilled into the PICC lumen, left for 10-60 minutes, and then flushed away with normal saline. If there is difficulty injecting urokinase, the POP technique can be used (Appendix 5) prior to insertion of urokinase. If the catheter cannot be unblocked, it will need to be removed. Diagnosing the cause of the occlusion is essential in order to treat the occlusion 
properly. Migration of the PICC inwards can sometimes be corrected without PICC removal but migration outwards cannot. A broken or damaged catheter normally needs to be removed, as it is contaminated.

\section{Documentation of complications}

Both minor and major PICC complications should be documented by nursing staff dealing with the PICC. Each week when the PICC dressing is changed, the nurse should also complete the Maintenance and Care Plan form (Appendix 6). This is found on the back of the PICC Patient Information form. Each week the nurse documents the PICC external catheter measurement to ensure the PICC has not migrated in or out. The nurse also records a phlebitis score and documents any other complications. If a complication has occurred, the Central Venous Access Device Record form should also be completed (Appendix 7). This form has an area to document complications. If the complication is major and requires early PICC removal, the Central Venous Access Record form should be completed. Section Three of the form has provision for the nurse to write particulars about removal such as date of removal, reason for removal and a 'Comments' area to write in more detail what the complication had been.

Information surrounding PICC insertion in oncology patients is entered onto the 'PICC/Midline Patient Information' form (Appendix 3) which is held by the patient but also photocopied with a copy held in the Oncology Outpatients' Department as per standard practice.

At each dressing change the catheter, insertion site and arm are examined for any complications. If the district nurses find any complications when dressing the PICC in the patient's own home, they send the patient into the Oncology Outpatients Department for further assessment, or the oncology ward if it is out of hours. Nurses on the ward or Oncology Outpatients' Department will then document any complications on the Central Venous Access Device Record form (Appendix 7). Any action taken is also documented on this form. Both these forms are filed in the Oncology Outpatients' Department as per usual practice. These forms were the main source of retrospective data for the present study. 
This chapter has described the clinical context where PICCs are inserted, managed and accessed within the oncology setting in which this study took place. PICC advantages for oncology patients were described. Inherent in this process, is a sense of professional responsibility to prepare the patient for management of their role in safe care of their PICC. All staff who care for and access the site require a high standard of education surrounding PICCs and IV treatments, and have followed the appropriate guidelines. In the next chapter (Chapter 4), this study's methodology and design are discussed. 


\section{Chapter Four: Methodology and study design}

\section{Introduction}

In order to ascertain the rates and incidence of peripherally inserted central catheter (PICC) line complications in oncology patients over a 13-month period, this study adopted an observational methodology with a retrospective design. It was intended that the nature of emergent complications would be explored, and a description of these be given. Furthermore, rates and complications would be analysed in such a way as to identify possible relationships between them. It was also expected that other variables might present themselves within the data collected, and these too would be described and the data analysed to establish whether there were differences between patients who had complications and those who did not.

The literature review presented in Chapter 2 revealed that there were three main complications from PICC lines, infection, venous thrombosis and mechanical failure. Previous studies have explored the rates and nature of complications in silicone (Groshong ${ }^{\mathrm{TM}}$ ) PICCs but not in polyurethane (Arrow ${ }^{\mathrm{TM}}$ ) PICCs, polyurethane PICCs being the catheter of choice at Christchurch Hospital. Furthermore, there was little research on PICCs in oncology patients per se. This study's intent was to not only contribute towards bridging this gap in the literature, but also to offer findings that might improve practice surrounding PICC use. Furthermore, a retrospective design was deemed appropriate as data were already being collected as part of patient care. What this study would do was go back and access that data, collate it, analyse it, and describe its findings.

\section{History of observational methodology and its philosophical underpinnings}

Observational methodology began in the early work of epidemiology where disease progression was monitored. Patterns and the nature of the disease process were observed by examining past epidemics (Matthews \& Venables, 1998). Epidemiology as a non-experimental methodology focuses on identifying and understanding causal pathways surrounding disease (Vandenbroucke, 2002). Before the 1890s epidemiology was by and large conducted by observing phenomena within its own environment. It focused closely on patterns of disease, but entered into little 
speculation about causes (Hardy \& Magnello, 2002; Vandenbroucke).

By the close of the $19^{\text {th }}$ century, many researchers had adopted simple statistical methods to make sense of data observed. Hence there was a move towards statistical epidemiology. Now findings could be measured by mathematics. Epidemiologists such as Greenwood, Ross, Yule and Pearson and Snow, Farr and Frost (Hardy \& Magnello, 2002) contributed strongly to the move towards statistical epidemiology. These early epidemiologists began collecting data systematically in order to look at patterns in order to establish what was happening within population groups. Furthermore, as the $20^{\text {th }}$ century got under way, epidemiologists were applying focused reasoning to what the relationships amongst the variables within the data might be. Hence they were using observation to describe variables within the data and statistics to measure those variables.

By the 1950s researchers were well and truly taking yet a further step within the research process. They used emergent findings to make predictions surrounding disease, its cause and progression. Thus speculations were made based on research findings. Eventually, statistics were applied to randomisation within populations as epidemiologists sought universal laws a priori surrounding disease (Hardy \& Magnello, 2002).

Observational studies are positioned within epidemiology in that the researcher is interested in determining how subjects might behave under certain conditions (Beanland, Schneider, LoBiondo-Wood, \& Haber, 1999). Peat (2001) categorises observational research alongside descriptive and non-experimental methodologies. These three research methods are particularly useful in describing rates of disease in a population group, identifying associations between variables, and can be either quantitative or qualitative in nature. Data are gathered via a cohort, case-control, cross-sectional, ecological, a case series or a case report (Ligthelm et al., 2007; Peat). Furthermore, all three methods generate hypotheses rather than testing them. Ligthelm et al. point out that observational studies are a key component in a balanced evidence base for clinical decision making as they play an important role in investigating treatment outcomes. In this philosophy it is asserted that phenomena do not randomly occur but have preceding causes, which can be deducted by detailed 
observation. This detailed observation can be reliably replicated and generalised to similar populations or conditions. The researcher remains objective in the enquiry/data collection process in order to remain an independent observer of the phenomena of interest. Thus the research can infer that a relationship may exist (Gillis \& Jackson, 2002).

Observational studies can utilise retrospective or prospective data. Peat (2001) explains that retrospective data are "collected using subjects' recall about illnesses or exposures that occurred at some time in the past or collected by searching medical records" (p. 17). Doll (2001) and Luepker (2005), also reiterate that data can be used retrospectively by exploring written records of past events. In contrast, prospective data are that which is, "collected about subjects' current health status or exposures as the study progresses" (Peat, p. 17). This study has a retrospective design, as the majority of the data utilised in this study were gathered in a systematic way at the time people were receiving care related to their PICCs. Staff were expected to routinely complete documentation. However, the data were not accessed by the researcher until after the PICC had been removed. When the researcher proceeded to enter the data into the Statistical Package for Social Sciences (SPSS) (Pallant, 2002), it was found that there were considerable data missing. Clinical records in addition to the PICC documentation needed to be searched for missing information.

This observational clinical study then, gathered data retrospectively from medical records on a cohort of oncology patients who had PICCs inserted for the administration of their cancer treatments. Crookes and Davies (1998, p. 313) define cohorts as, "samples in time-dimensional studies within the field of epidemiology". The data gathered were analysed quantitatively by utilising the widely recognised SPSS, Version 14. The study sought to describe the complications that occurred whilst the PICC was in situ, as well as the rates of those complications. Tentative relationships between emergent variables, for example, differences between patients that had complications and those that did not, were also of interest to the researcher.

\section{Sample}

All adult oncology patients (excluding Haematology and Bone Marrow cancer patients), who had a PICC line successfully inserted during a 13-month period from 
March $1^{\text {st }} 2006$ to March $31^{\text {st }} 2007$ at Christchurch Hospital, were retrospectively identified. This was done by accessing all of the PICC/Midline Patient Information forms (Appendix 3) filled in during the 13-month period. Each PICC insertion was documented on its own form. The original became part of the clinical notes and a photocopy of each insertion was sent to the Oncology Outpatients' Department, where initial follow-up occurs. Once identified as having had a PICC insertion and meeting inclusion criteria for the oncology cohort, further clinical records were accessed for the express purpose of gaining retrospective data. These included, follow-up forms (Appendices 6 \& 7), doctor's letters, and patients' clinical notes. Inclusion criteria for this research were all oncology patients within the Canterbury District Health Board (CDHB) Oncology Service who had a PICC line inserted for their cancer treatment. Exclusion criteria included anyone who was unable to be followed over that period in retrospective follow-up.

\section{Background and development of forms}

The forms used to extract the research data (Appendices 3, 6, \& 7) had been in existence since an earlier aborted attempt to undergo a retrospective study. That study was abandoned due to inadequate documentation on the clinical forms. Key information had either been left out or incompletely recorded. For these reasons, nursing practice related to documentation was addressed. Change was needed and action was taken at that time to ensure that nurses became more vigilant at completing all documentation. The researcher had been one of the drivers in this process. Staff were informed and encouraged to complete the forms more fully. On these grounds, it was anticipated that any subsequent retrospective study would provide sufficient data to ascertain the complications surrounding PICC insertion and management, the rates of those complications and any possible relationships between them.

Three forms were designed to track patients' insertion and follow-up care of their PICC. Aspects listed on each of the forms to prompt information gathering was guided by a previous literature review as well as clinical needs. The 'PICC/Midline Patient Information' form (Appendix 3) included demographic patient particulars (Name, DOB, age, address, hospital number), insertion details, catheter type, reason 
for insertion, allergies, instructions for flushing the PICC and information regarding the nature of the catheter inserted. The 'Central Venous Access Device Record' form (Appendix 7) also included insertion details (which nurses transferred from the 'PICC/Midline patient Information' form). It included complications at insertion, catheter position, external catheter measurement, patient education, complications and removal details. The options under the 'complications' section of the form were, difficulty with flushing, haematoma, phlebitis, haemorrhage, and thrombus. Appraisal of the form's content is discussed further in Chapter 6.

\section{Method}

As part of normal documentation processes, the relevant PICC forms (Appendices 3, $6, \& 7$ ) were photocopied and the copies were placed in a separate file and kept in the Oncology Outpatients' Department. Once the cohort eligible for the study were identified, information recorded on the forms were extracted and entered into a database using SPSS. This package was chosen as it is widely recognised as a useful statistical package for computer-based data analysis in Social Science research.

\section{Data collection}

Key aspects in this study were retrieving information recorded on the PICC line documentation forms. The information collected on these forms and the collection process is routine practice. As part of usual practice, an Oncology Outpatients' Nurse collated, photocopied and filed the data forms as they came to hand. Thus collated files were stored in the Oncology Outpatients' Department. To facilitate completeness of data it was expected as part of the usual record-keeping surrounding these forms, that the Oncology Outpatients' Nurse would also be responsible for filling in gaps on the forms.

Following a previous study that was aborted due to significant amounts of missing data, but prior to the current study, steps were undertaken by the department to increase the completeness of data collection, and included a close initial monitoring of the form-filling to identify problem areas. This revealed for example that patients who were being admitted with PICC complications as inpatients were sometimes not having this information added to the Oncology Outpatients' Nurse's file. To address 
this issue, the ward Clinical Nurse Specialist faxed relevant ward information to the Oncology Outpatients' Department Nurse. This responsibility was then broadened to include all staff on the ward. It became each nurse's responsibility to put the patient's sticky label on a blank piece of paper with as much relevant information about the PICC complication as possible, and then fax this to the Oncology Outpatients' Department Nurse. This was undertaken in order to capture all PICC complication data. Education about this new responsibility was documented in the ward communication book, along with discussions and explanation at ward meetings, whilst regularly encouraging and thanking staff for maintaining focus on the appropriate documentation. As explained previously, the nurse in the Oncology Outpatients' Department who had the responsibility to file the forms played an added role in improving this process by finding out missed information and adding it to the form.

Thus, all PICCs inserted for the 13-month period should have had forms residing in the Oncology Outpatients' Department in a file. Any PICCs still in place at the end of this 13-month period were followed for a further three months.

Data were entered by the researcher into the statistical computer programme, SPSS in the Oncology Outpatients Department. The dwell time of the PICC line was manually calculated as were the number of days to complication. At this point the researcher attempted to obtain any missing data by going back to the patient file to observe whether the missing information was documented there. This proved to be successful in several cases and entailed about 40 patient files. If the exact date of removal could not be elicited, the date of removal was conservatively estimated as the next day after the PICC line was last reviewed, which was in line with the most recent published study on PICC lines in oncology patients by Yap et al. (2006). The literature review did not elicit any studies where missing data were discussed except one, where the patients who were lost to follow-up were withdrawn from the study altogether (Walshe et al., 2002). All relevant computer data were backed up twice a week onto a memory stick in order to keep the information secure. 


\section{Analysis}

The SPSS programme was utilised for the statistical analyses. Following entering and cleaning the data to confirm that it had been entered accurately, a three step analysis plan was adopted.

Step one involved describing the participants and determining baseline data including demographic information such as gender, type of cancer, planned therapy type, and catheter details. Measures of central tendency such as mean, median and mode and measures of dispersion such as standard deviation, inter-quartile range and frequencies were used depending on variable type. Means and medians were used to describe the continuous variables such as age. Frequencies were used for nominal variables such as region and type of cancer. Standard deviations were obtained to determine data distribution. In carrying out this step, individuals who had a PICC line inserted on more than one occasion were only counted once - the first time they had a PICC line inserted. Results were assessed to ensure that they were not skewed. This step was paramount as the subsequent analysis depended on it.

Step one also involved some recoding and regrouping of variables. As part of this regrouping, thought surrounded colorectal cancers. Normally these cancers would be classified as gastro-intestinal; however the colorectal cancer group was large enough to place within a category of its own. Thus these two cancer sites have been separated. Hence gastro-intestinal includes oesophageal, gastric, pancreatic, liver, jejunum, gallbladder, and hepatobiliary cancers and colorectal includes small bowel, caecal, appendix, sigmoid colon, rectal, and anal cancers. A further regrouping of the values concerned where the patient resided. Given the diverse range of geographical areas within the cohort, this data were clustered into six groups. They were Canterbury, Blenheim, Nelson/Marlborough, West Coast, Timaru, and Kaikoura. These six groups were regrouped again into two groups, Canterbury (including Timaru and Kaikoura) and Outer Region (including Blenheim, Nelson/Marlborough, and West Coast).

Step two involved describing the number and types of complications and establishing the rate of complication by number of insertion days. Yap et al. (2006) describe 
expressing PICC complication rate as complication per 1000 PICC days in order to compensate for people who only require PICCs for a short time. The rate of catheter complications was determined per 1000 catheter dwell days and calculated as follows: the total number of complication events divided by the total number of device days and multiplied by 1000. In carrying out step two all PICC line insertions were included irrespective of whether a person had had one previously. It also involved calculating the dwell time of each PICC line and then the number of days PICC lines were free of complications.

Step three used inferential statistics such as chi square, t-test and Fisher Exact Test to establish whether there were any demographic or clinical differences between those with complications and those without. These complications included infection, mechanical failure and venous thrombosis amongst others. Statistical difference was expressed as the $p$ value being less than or equal to 0.05 . The statistic of choice was determined by the data distribution and cell size. Where the cell size was fewer than five, the Fisher Exact test was used.

\section{Rigour}

Rigour concerns the scientific quality of the research design and it involves validity, reliability, objectivity, and generalisability (Mathers \& Huang, 1998; Peat, 2001). Von Elm et al.'s (2007) framework for reporting observational studies informs how this thesis has been conducted and written. This framework offers clear direction in how to proceed with the research process using an observational methodology. Each item outlined in the framework is fully addressed in the body of work. This adds a great deal of strength to this study. How validity, reliability, objectivity and generalisability were managed within this study will now be discussed.

\section{Validity}

Validity is the degree to which the results indicate measurement of what is supposed to be measured within the research (Beanland et al., 1999; Peat, 2001; Polit, Beck, \& Hungler, 2001). It also estimates the accuracy of the instrument chosen to elicit the data (Peat). Validity is measured both internally and externally. Internally, a key focus of this study centres on the forms used to collect data on the insertion and 
monitoring of PICC lines in oncology patients. Mathers and Huang (1998) refer to 'face' or 'content validity'. "This relates to whether 'on the face of it' the instrument or study measures what it is supposed to measure" (p. 145). In the practice setting currently the forms are used because 'on the face of it' they will give the information that will be useful for practice, and the researcher needed to be confident that the forms captured the information about those involved in the study and what happened to their PICCs. The research built on the assumption that staff had a shared definition of what was a complication. A consistent approach to decision-making when a person developed two related complications was also required.

The definition of whether a complication was minor or major, and what constituted one complication was consistently applied throughout the research. Two PICC lines developed a second complication but these were counted by the researcher as one complication, for the following reasons. The first PICC line developed infection as a complication, and then three days later developed thrombosis. At this point, the PICC line was removed. These two complications are known to be related as discussed in Chapter 2, so were therefore counted as one complication. The other PICC line had a minor skin reaction as the first complication, followed by a major skin reaction. The PICC had been scheduled for removal the day after the major skin reaction anyway, so was removed one day early. This was counted as one complication in this study because the first complication was a minor complication and therefore not the topic of this study.

Essentially then for the context where this study took place, this research is entirely valid. This thesis attempted to reflect a level of internal validity by also giving detailed and accurate descriptions of the setting and research participants as is recommended by Mathers and Huang (1998) and von Elm et al. (2007). Furthermore, the researcher was the only one who collated the data and entered it into the SPSS package. The entry process was checked by her thesis supervisor to strengthen accuracy and thus it can be argued that this contributed to internal validity.

Externally, validity entails correct selection of participants or subjects that can as accurately as possible give valid contributions to the research (Peat, 2001). Steps were taken to ensure all eligible patients were entered into the study. In this study 
patients were retrospectively identified as those that were oncology patients, who had PICCs inserted for their treatment. Each patient had three forms that were being used to monitor both the insertion procedure and occurrence of PICC complications. Should other contexts choose to have the findings of this study inform their own practice setting, they will clearly know which group the findings might apply to.

\section{Reliability}

Reliability can be defined as the extent to which the results would be the same if the research were repeated under similar circumstances. It seeks to signal the amount of error, random and systematic, implicit in any measurement (Burns \& Grove, 2001). In this research this mainly concerns the reliability of the data collection tools. Although not formally pre-tested, the forms were audited prior to the current study in response to the earlier aborted research project. Although no specific changes were made to the forms at that time, this audit process was done to increase the likelihood of staff fully documenting PICC-related actions at the time of a patient consultation. This auditing and the improvement in the systematic data collection process indicated steps taken by the institution. This assisted the researcher in strengthening reliability of the findings.

Reliability of the proposed study may have been strengthened by a concurrent project using an oncology group within another setting. This was not feasible due to time, fiscal and research thesis size constraints. It may be possible to replicate and broaden the study at a later date. So, this study is reliable for the context in which it is positioned and can be relied upon to inform other practice areas who wish to explore PICC complications within their oncology population.

To further add to reliability, oncology patients who still had PICCs in situ at the study's end were followed for another three months (until $31^{\text {st }}$ July 2007) or until their PICC was removed, whichever came first. This was done in order to allow further time for potential complications to emerge surrounding these PICCs. There were four PICCs whose exact removal date could not be accurately ascertained, so the removal date for analysis was conservatively taken to be the day after the last documented review, in keeping with Yap et al. (2006). 


\section{Objectivity}

Burns and Grove (2001) indicate that objectivity is achieved when the findings are free of researcher bias, values or prejudice. Peat (2001) further argues that subjective data are that which is gathered on a questionnaire and that objective data would be gained from instruments that are less open to interpretation or influence by the subject or the observer. In this study, all data gathering forms were a list of prompts (Appendices 3, 6, \& 7) where the nurse filled in the data in response to the prompt. Furthermore, a section named 'other' provided nurses with a place to record unexpected complications, thus not limiting possible answers. The researcher was open to whatever findings presented themselves and attempted to remain free from bias by being true to the data. As all the data were collected prior to analysis the researcher did not know what was to be found until the data were analysed.

Peat (2001) discusses responsiveness within the research process. The author explains that the main outcome of measurement is to be responsive to differences between subjects and/or changes that occur within a subject. Central to this study is a concern for improving practice, wellbeing and quality of life for those within the population who have cancer. Responsiveness seems wholly appropriate. For this study, data gathered retrospectively can be free from researcher bias in that the data are already documented by someone else - the nurse inserting the PICC or the nurse monitoring follow-up care. Or, if the data were missing the Oncology Outpatients' Nurse during usual documentation processes, requested the information so that this nurse could write it on the form. Also, as the researcher retrospectively accessed the data, she then went back to the clinical notes to find the information and write it on the form. In this way data were added verbatim to the forms from the clinical notes. Furthermore, as the researcher worked with the data, she made decisions around where to place the data prior to SPSS entry and also she described existing and emergent variables that presented themselves in the analysed data. The process used for this was structured and where the researcher made decisions that could have impacted on results, for example whether a complication was one or two complications, a documentation trail was created to indicate the basis for the decision. 


\section{Generalisability}

The question of generalisability looks at which populations, settings, treatment variables and measurement variables the results can be generalised to. Generalising across persons requires research samples to be representative of the population of interest. Tests that meet representativeness criteria are, in essence, tests of statistical interaction (Cook \& Campbell, 1979). In identifying complications and their rates within PICC lines in oncology patients it has been a challenge for this study to emerge with sufficient statistical significance to generalise to other situations. However, the study is deemed worthy in that it would potentially identify variables that could be considered in other contexts so that nurses can more thoughtfully plan care. Furthermore, representativeness is the extent to which the sample matches the target population (Burns \& Grove, 2001). For this study the sample group was adult oncology patients, so only adult oncology patients who had a PICC inserted within the given timeframe were tracked. Thus the study was fully representative of the intended group.

In terms of external generalisability, all cancer centres are different. This study's oncology population had almost all solid tumours and a high percentage of patients with colorectal cancers. It also has limited generalisability to entire oncology populations in that the results cannot be applied to those patients with haematological cancers.

\section{Ethical considerations}

Ethics (from Greek) essentially means the rights and wrongs of human behaviour and refers to the science or study of morals (Thompson, Melia, \& Boyd, 1996). When conducting nursing research, the moral principles to consider include autonomy (patient preferences be respected and informed consent be given), non-maleficence (that the researcher 'do no harm'), beneficence (that benefit be bestowed), and justice (the right to be treated fairly and with respect) (Johnstone, 1994).

In this study, informed consent to have a PICC inserted is gained prior to insertion. The Agreement to Treatment form is used to gain this. Furthermore, within Christchurch Hospital, patients who are offered chemotherapy as part of their 
treatment also sign a consent form which refers to the fact that data are collected for audit purposes. The researcher communicated with the local ethics committee for guidance as to whether separate consent to be included in the study was required. It was established by the committee that as this was a retrospective study utilising clinical data that was routinely recorded, individual consent was therefore deemed not necessary. The data were part of the normal audit and tracking processes of the hospital involved. All responsible hospitals are constantly collating and auditing infection control information and statistical data from the interventions that are performed there.

This study was considered an extension of this audit process, where a specific group of patients (oncology patients) received a specific intervention (PICC line insertion), and the complications were tracked, using existing clinical data. The information gathering did not impact on the patients at all because it was data that was normally recorded by staff as a matter of course after PICC insertion, flushing, education or management.

Another important ethical consideration is the protection of the participants' rights to confidentiality, anonymity, and privacy. During the research process, all data were collected using National Health Index (NHI) number only (no names recorded) and then each person was given a research number and the NHI number was removed from the file being analysed. This computer required use of a secure password in order to access any data, thus maintaining confidentiality of the information. Home and work based security systems were used to minimise the chance of theft and access to the data. Only the researcher, the Outpatients' Oncology Nurse, the statistician, and the oncologist interested in pursuing this study and the researcher's academic supervisor had access to the data.

The study received approval from the Upper-South Regional Ethics Committee in February 2007 (Appendix 1). Overall then, the study was deemed ethically sound, benefiting both the patients and the institution. 


\section{Treaty of Waitangi responsibilities}

The researcher was mindful that in New Zealand the Treaty of Waitangi always applies in nursing research. Furthermore, currently as at February 2008, in all CDHB position descriptions for employment it is stated: "The Canterbury District Health Board is committed to the principles of the Treaty of Waitangi and overarching objectives of the New Zealand health and disability strategies." Furthermore, the Code of Health and Disability Services Consumers' Rights outlines patients' rights surrounding informed consent. Thus, it can be assumed that informed consent was given by the patient for a PICC to be inserted for treatment purposes.

Part of Treaty of Waitangi principles incorporate the concept that hospitals are representatives of the Crown and thus researchers and health professionals need to take extra care that what they do and how they work with the patient is culturally safe for that patient. In this study, the researcher did not know if Maori were involved in this study because ethnicity data are not recorded on the form. It is possible that ethnicity data should be recorded on the form at some stage in the future. In the meantime, it can only be assumed that staff involved with the care of the patients in this study had adhered to legal and ethical requirements in regard to patients' rights and culturally safe care.

\section{Summary}

Few studies exploring PICC complications in oncology patients exist. To ensure best practice surrounding PICC insertions, management and treatment delivery, it was deemed important that valid research outcomes inform practice. To this end, this study adopted an observational methodology with a retrospective design to explore the incidence and rates of PICC complications in a typical adult oncology cohort over a 13-month time span. Usual audit and documentation were to be retrospectively examined and this data be entered into a statistical package for analysis. It was intended then, that valid findings could positively impact on patient outcomes in the practice setting. Furthermore, it was anticipated that the design of this study might be replicated in other cancer settings. To conclude then, each step of this research process was underpinned by methodological and ethical principles in order to safeguard the patients involved, the researcher and the integrity of the 
research findings. In the following chapter (Chapter 5), the findings of this study are presented. In Chapter 6 those findings are discussed, related to wider literature on the topic, and recommendations for practice are proposed. 


\section{Chapter Five: Results}

\section{Introduction}

This chapter describes the sample population and presents the findings of a 13-month observational study in which the incidence and rates of peripherally-inserted central catheter (PICC)-related complications amongst oncology patients are explored. All adult oncology patients from one regional centre who had a PICC inserted between 1st March 2006 and 31st March 2007 were retrospectively identified and entered into this study. Although it had been initially planned to also include paediatric oncology patients, these patients were excluded from this study when it was revealed that they were managed and treated through a different department (not the oncology department in which this study is based), with differing documentation trails.

\section{The sample}

One hundred and sixty five PICC lines were inserted into 157 oncology patients during the 13-month period of the study. One patient moved out of the research area and it was therefore not possible to access retrospective data. They were withdrawn from the study by the researcher prior to data analysis, thus the research involved 156 oncology patients and 164 PICC lines. Of the 156 patients, 148 (95\%) had one PICC line inserted, seven (4\%) had two, and one patient (1\%) had three PICC lines inserted over the 13-month period. Clinical and demographic data of the 156 patients are outlined in Table 4 below. Data gathered such as age and diagnosis, were taken from the first PICC line inserted for each individual patient. Thus Table 4 reflects demographic and clinical features of the 156 patients involved in the study. In contrast, some tables and analyses subsequent to this, count each PICC placement as a new event, reflecting analysis of the 164 specific PICC line insertions in order to analyse the data more accurately. This keeps this study in line with other similar published studies.

The mean age of the patient population was 62 years (standard deviation (sd) 11; range, 23-79 years). Of this cohort, 20 patients were under 50 years of age (13\%) with the majority of patients over 50 years of age $(87 \%$ or $133 / 156)$, a feature which may be predicted within an adult oncology population. Overall, the cohort included 
Table 4. Demographic and clinical features of the 156 patients with PICC lines

\begin{tabular}{|c|c|c|}
\hline Characteristic & Values & No (\%) \\
\hline \multirow[t]{5}{*}{ Age } & $\leq 40$ & $7(4 \%)$ \\
\hline & $41-50$ & $16(10 \%)$ \\
\hline & $51-60$ & $48(31 \%)$ \\
\hline & $61-70$ & $45(29 \%)$ \\
\hline & $>70$ & $40(26 \%)$ \\
\hline \multirow[t]{2}{*}{ Gender } & Male & $87(56 \%)$ \\
\hline & Female & $69(44 \%)$ \\
\hline \multirow[t]{2}{*}{ Region } & Canterbury & $134(86 \%)$ \\
\hline & Outside Canterbury & $22(14 \%)$ \\
\hline \multirow[t]{8}{*}{ Underlying cancer site } & Colorectal & $110(71 \%)$ \\
\hline & Gastro intestinal & $20(13 \%)$ \\
\hline & (excluding colorectal) & \\
\hline & Breast & $16(10 \%)$ \\
\hline & Lymphoma & $2(1 \%)$ \\
\hline & Ovarian & $3(2 \%)$ \\
\hline & Other & $3(2 \%)$ \\
\hline & Not Stated & $2(1 \%)$ \\
\hline \multirow[t]{2}{*}{ Underlying cancer type } & Solid tumour & $154(99 \%)$ \\
\hline & Haematological tumour & $2(1 \%)$ \\
\hline
\end{tabular}

$87(56 \%)$ men and $69(44 \%)$ women and thus demonstrates a slight male predominance. All 156 patients lived within the geographical area in which the Canterbury District Health Board's Oncology Department has responsibility for patient cancer treatment and care. More specifically, $134(86 \%)$ lived in the Canterbury region, 12 (7\%) lived in the Nelson/Marlborough region, and $10(6 \%)$ lived on the West Coast of the South Island.

Almost all patients (154 or 99\%) had a solid tumour. As Table 4 shows, the largest group according to underlying cancer site was the colorectal cancer group (71\%). The colorectal and gastro-intestinal cancer patient groups combined comprise a very 
high $84 \%$ of this study's cohort, reflecting the frequent use of infusional chemotherapies in these cancer subtypes. Fifty-five percent of patients had metastatic disease at the time of PICC insertion, and 1\% had probable metastases.

\section{PICC line insertion and management information}

The retrospectively accessed documentation indicates that the reasons the 156 patients had PICC lines inserted did not vary from the first to the second insertion. Table 5 indicates the main reason for patients having a PICC line. The majority (at least $83 \%$ ) of patients had a PICC line primarily inserted for chemotherapy. It is very likely that PICC access for patients with poor venous access and needle phobia was also in order to administer chemotherapy, but this was not specifically recorded in the documentation.

Table 5. Indicators for PICC insertion

\begin{tabular}{|l|r|}
\hline Reason & No.(\%) \\
\hline Continuous infusion & $97(62 \%)$ \\
\hline Intermittent infusion & $33(21 \%)$ \\
\hline Poor venous access & $25(16 \%)$ \\
\hline Needle phobia & $1(1 \%)$ \\
\hline Total & $156(100 \%)$ \\
\hline
\end{tabular}

A single-lumen catheter was inserted in $115(70 \%)$ of 164 PICC placements, and a double-lumen catheter was inserted in 49 cases (30\% of the sample). No triple-lumen catheters were inserted over this period. All single-lumen catheters were size four French and all double-lumen catheters were size five French. Internal length and external length of the catheter varied from $30-51 \mathrm{cms}$ and from $4-21 \mathrm{cms}$ respectively, depending on the size of the patient (Chapter 3 provided an explanation as to how catheter length was established for patients). No catheters were trimmed in length at insertion and thus all remained at $55 \mathrm{cms}$ in length.

Within the research catchment area, it is common practice for specially-trained nurses to insert a PICC line into the patient's non-dominant arm to reduce the risk of 
overuse complications such as phlebitis. Hence it is no surprise that $128(78 \%)$ PICCs were placed in the patient's left arm, and 35 (21\%) were placed in the patient's right arm, reflecting the predominance of right-handedness in the community. Documentation surrounding the insertion of only one of the PICC lines did not state whether handedness was ascertained and considered prior to PICC insertion.

As stated in Chapter 3, the basilic vein is considered the most appropriate vein to use for PICC insertion. This is also reflected in the findings below. The basilic vein was used for $146(89 \%)$ PICC insertions, the brachial vein for three (2\%) insertions, the cephalic vein for four (2\%) insertions, the median vein in one $(0.6 \%)$ PICC insertion, and the vein used for insertion was not stated in $10(6 \%)$. All PICCs were placed above the antecubital fossa, as is considered best practice (see Chapter 3). Thirty-two (20\%) patients had a known allergy and $128(78 \%)$ did not, and data were missing for four (2\%) PICCs. Of the 32 patients who had a known allergy, there were a vast array of drug and other allergies. Ten patients were allergic to penicillin. Other allergies ranged from intravenous (IV) contrast, IV3000 ${ }^{\mathrm{TM}}$ dressings, to bees, shellfish and sulphur drugs. No-one was documented as having previous allergies to catheters or polyurethane. No patients were administered antibiotics at PICC insertion, according to PICC insertion notes.

Six (4\%) patients had complications described at insertion, none of which required premature PICC removal. One $(0.6 \%)$ patient sustained a haematoma at insertion and five other insertions were described as a "difficult insertion". Only 25 (15\%) records had ongoing weekly documentation about their PICC follow up care completed. The other $139(85 \%)$ records had incomplete documentation. Documentation that the formal patient education about PICCs and their use was given was indicated in 120 (73\%) patients, five (3\%) patients did not receive PICC education prior to insertion, and whether patient education had occurred was not indicated for 39 (24\%) patients. Of the 156 individual patients 120 had formal education at least once.

The person trained to be the primary person to flush the PICC differed as follows: 23 (14\%) were patients, $15(9 \%)$ were relatives, $29(18 \%)$ were district nurses, and oncology nurses flushed the PICC for 71 (43\%) patients. Data were missing on 26 
(16\%) patients. Oncology nurses flushed the PICC due to infusional chemotherapies. A total of $85(52 \%)$ patients were referred to the district nursing service mainly for weekly dressings, and also for daily flushing of the line for 29 patients. Forty-four (27\%) patients were not referred to district nurses, probably due to their PICC being managed by oncology nurses, and the data on this variable was missing for $35(21 \%)$ patients.

The 164 PICCs were in place for a total of 14,276 catheter-days. The median dwell time was 68 days (range 6 - 412 days). The interquartile range was 39 - 126. An additional analysis of the data were undertaken excluding the person who had 412 catheter-days in situ because the length of PICC dwell time was 139 days more than the next person and this analysis obtained a median of 67.0 days (range 6 -273), with an interquartile range of 39 - 125 days. Given the findings of this outlier analysis all future analyses included this PICC. At the end of the 13 month period, seven (4\%) PICCs were still in situ, and each still had the same PICC in place at the end of 3 months. These seven PICCs were counted as removed on this date when analysing the data. The exact date of PICC removal for four patients was unclear, and in keeping with Yap et al. (2006) the removal date for analysis was taken to be the day after the last documented review, a conservative analysis.

Reasons for the removal of the 157 PICCs are listed in Table 6.

Table 6. Reasons for PICC line removal

\begin{tabular}{|l|r|}
\hline Reason for PICC removal & N (\%) \\
\hline Completion of therapy & $97(61.7 \%)$ \\
\hline Disease progression & $26(16.6 \%)$ \\
\hline PICC complication & $16(10.2 \%)$ \\
\hline Death & $7(4.5 \%)$ \\
\hline chemotherapy complications & $5(3.2 \%)$ \\
\hline Patient request & $2(1.3 \%)$ \\
\hline Other & $4(2.5 \%)$ \\
\hline
\end{tabular}


This table excludes the seven people who still had the PICC in situ. From this table it is apparent that just under two thirds (61\%) of PICC lines were removed because of completion of therapy, and $21(13 \%)$ were removed because of either chemotherapy or PICC complications.

\section{Complications}

Given that the outlier analysis did not make a notable difference in the total number of PICC days, the analysis on rates was undertaken on the 14,276 days. Within this study, PICC complications developed in 25 (15\%) out of 164 PICC lines, in 22 (15\%) of the 156 patients. However, only 16 (64\%) of the 25 PICCs with complications required early removal (which equates to $10 \%$ of the total number of PICCs), the overall complication rate was 1.75 per 1,000 catheter-days, and for complications leading to early removal was 1.12 per 1,000 catheter-days. The mean time for a complication to occur was 51.8 days (sd 37 days), with a median of 41 days (range of 2 - 160) days and an interquartile range of 24.75 - 76.50. No PICC lines were assessed to have more than one complication. However, in one case where the patient had thrombosis followed by infection, a decision was made that this would be counted as one complication and that the cause would be infection. If this had been counted as two complications, then the final number of complications would have been higher. In another case where the patient presented with the complication of minor skin reaction, and then later presented with a complication of major skin reaction, a decision was made to count this as one complication because the first complication was considered a minor complication and therefore not part of this study. No patients required intensive care treatment or died as a result of PICCrelated complications over the period of this study.

Infection was the most common complication at $4.3 \%(7 / 164)$ and equates to 0.49 infection complications per 1000 PICC-days. This was followed by PICC migration at $3 \%(5 / 164)$ which equates to a PICC migration complication rate of $0.35 / 1000$ catheter days, and thrombosis at 2.4\% (4/164) for a rate of thrombosis complication of $0.28 / 1000$ catheter days. Other PICC-related complications included three (1.8\%) patients with a major skin reaction for a rate of $0.21 / 1000$ catheter-days, two (1.2\%) 
patients with difficulty flushing their PICC at a rate of 0.14 complications per 1000 catheter-days, two $(1.2 \%)$ patients with phlebitis for a rate of $0.14 / 1000$ catheterdays, one $(0.6 \%)$ patient with PICC occlusion for a rate of 0.07 per 1000 PICC-days, and one $(0.6 \%)$ patient with accidental removal of their PICC for a rate of $0.07 / 1000$ catheter-days. Table 7 presents the reasons for complications and the corresponding rates per 1,000 days.

Table 7. PICC complications by number, percent and rates

\begin{tabular}{|l|r|r|r|}
\hline $\begin{array}{c}\text { Type of PICC } \\
\text { Complication }\end{array}$ & $\begin{array}{c}\text { No (\%) of 25 } \\
\text { PICC } \\
\text { Complications }\end{array}$ & $\begin{array}{c}\text { Rate per 1,000 } \\
\text { catheter days }\end{array}$ & $\begin{array}{c}\text { No (\%) of } \\
\text { complications } \\
\text { as proportion } \\
\text { of 164 PICCs }\end{array}$ \\
\hline Infection & $7(28 \%)$ & 0.49 & $7(4.3 \%)$ \\
\hline PICC migration & $5(20 \%)$ & 0.35 & $5(3.0 \%)$ \\
\hline Vein thrombosis & $4(16 \%)$ & 0.28 & $4(2.4 \%)$ \\
\hline Major skin reaction & $3(12 \%)$ & 0.21 & $3(1.8 \%)$ \\
\hline Phlebitis & $2(8 \%)$ & 0.14 & $2(1.2 \%)$ \\
\hline Difficulty flushing & $2(8 \%)$ & 0.14 & $2(1.2 \%)$ \\
\hline PICC occlusion & $1(4 \%)$ & 0.07 & $1(0.6 \%)$ \\
\hline Accidental removal & $1(4 \%)$ & 0.07 & $1(0.6 \%)$ \\
\hline
\end{tabular}

\section{Differences between patients and PICCs with and without a complication}

Findings of the analysis to identify whether there were any differences in those who did or did not experience a complication revealed that there were no statistical difference on any of the variables with the exception of cancer site. Table 8 reports these findings by the 156 patients and Table 9 by the 164 PICCs. The only variable with significant findings was that of the underlying cancer site. Those with complications were more likely to have a gastro-intestinal or an ovarian cancer diagnosis, and less likely to have a diagnosis of colorectal cancer. All those with an ovarian cancer as their primary site developed complications; however this entailed only five PICCs, and three individual patients. In other words, two individual patients with ovarian cancer had two different PICC lines inserted over this period both of which developed complications requiring premature removal. Another 
ovarian cancer patient had one PICC which required premature removal due to a complication. No ovarian cancer patient had their PICC removed because of thrombosis, which is a known risk for ovarian patients.

Table 8. Demographic and clinical features of the 156 by presence/absence of complications

\begin{tabular}{|c|c|c|c|c|}
\hline Variable & Values & $\begin{array}{c}\text { No } \\
\text { Complication } \\
\mathbf{n}=134\end{array}$ & $\begin{array}{c}\text { Complication } \\
\quad \mathbf{n}=22\end{array}$ & Significance \\
\hline Age & Mean (SD) & $62.28 \pm 10.7$ & $58.55 \pm 11.6$ & $\mathrm{t}=.103, \mathrm{p}=0.749$ \\
\hline Gender & $\begin{array}{l}\text { Male } \\
\text { Female }\end{array}$ & $\begin{array}{l}74(55 \%) \\
60(45 \%)\end{array}$ & $\begin{array}{r}13(59 \%) \\
9(41 \%)\end{array}$ & $X^{2}=115, d f=1, p=0.735$ \\
\hline Region & $\begin{array}{l}\text { Canterbury } \\
\text { Not Canterbury }\end{array}$ & $\begin{array}{r}114(85 \%) \\
20(15 \%)\end{array}$ & $\begin{array}{r}16(73 \%) \\
6(27 \%)\end{array}$ & Fisher Exact $p=0.212$ \\
\hline $\begin{array}{l}\text { Underlying } \\
\text { cancer site }\end{array}$ & $\begin{array}{l}\text { Colorectal } \\
\text { Gastro intestinal* } \\
\text { Breast } \\
\text { Lymphoma } \\
\text { Ovarian } \\
\text { Other } \\
\text { Not Stated }\end{array}$ & $\begin{array}{r}97(72 \%) \\
16(12 \%) \\
14(10 \%) \\
2(1 \%) \\
0(0 \%) \\
3(2 \%) \\
2(1 \%)\end{array}$ & $\begin{array}{r}13(59 \%) \\
4(18 \%) \\
2(9 \%) \\
0(0 \%) \\
3(14 \%) \\
0(0 \%) \\
0(0 \%)\end{array}$ & Fisher Exact $p=0.001$ \\
\hline $\begin{array}{l}\text { Cancer } \\
\text { metastases }\end{array}$ & $\begin{array}{l}\text { Yes } \\
\text { No } \\
\text { Probable } \\
\text { Not Stated }\end{array}$ & $\begin{array}{r}77(57 \%) \\
51(38 \%) \\
3(2 \%) \\
3(2 \%)\end{array}$ & $\begin{array}{r}9(41 \%) \\
12(54 \%) \\
0(0 \%) \\
1(5 \%)\end{array}$ & Fisher Exact $p=0.780$ \\
\hline
\end{tabular}

*Excluding Colorectal

With regards the metastases data, some patients who had more than one PICC inserted over this 13-month time period showed no mestastatic spread at first PICC insertion but did show metastatic spread when a second PICC was inserted at a later point. Therefore, these results report metastases in each patient at any point over the period of the study. There was no statistical difference between those patients with known metastases at time of PICC insertion and those with no metastases. 
Table 9. PICC details by presence/ absence of complications $(\mathrm{N}=164)$

\begin{tabular}{|c|c|c|c|c|}
\hline Variable & Values & $\begin{array}{c}\text { No } \\
\text { Complication } \\
\mathbf{n}=139(\%) \\
\end{array}$ & $\begin{array}{c}\text { Complication } \\
n=25(\%)\end{array}$ & Significance \\
\hline \multirow[t]{4}{*}{ Insert reason } & Continuous Inf & $91(65 \%)$ & $11(44 \%)$ & \multirow{4}{*}{ Fisher Exact $p=0.095$} \\
\hline & chemotherapy & $28(20 \%)$ & $6(24 \%)$ & \\
\hline & Poor access & $19(14 \%)$ & $8(32 \%)$ & \\
\hline & Patient & $1(1 \%)$ & $0(0 \%)$ & \\
\hline \multirow[t]{3}{*}{ Arm } & Left & $110(79 \%)$ & $18(72 \%)$ & \multirow{3}{*}{ Fisher Exact $p=0.516$} \\
\hline & Right & $28(20 \%)$ & $7(28 \%)$ & \\
\hline & Not Stated & $1(1 \%)$ & $0(0 \%)$ & \\
\hline \multirow[t]{5}{*}{ Vein } & Basilic & $122(88 \%)$ & $24(96 \%)$ & \multirow{5}{*}{$\begin{array}{l}\text { Not able to perform given } \\
\text { high number of 'not } \\
\text { stated' }\end{array}$} \\
\hline & Brachial & $2(1 \%)$ & $1(4 \%)$ & \\
\hline & Cephalic & $4(3 \%)$ & $0(0 \%)$ & \\
\hline & Median & $1(1 \%)$ & $0(0 \%)$ & \\
\hline & Not Stated & $10(7 \%)$ & $0(0 \%)$ & \\
\hline \multirow[t]{2}{*}{ Lumen } & Single & $96(69 \%)$ & $19(76 \%)$ & \multirow[b]{2}{*}{$X^{2}=.486, d f=1, p=0.636$} \\
\hline & Double & $43(31 \%)$ & $6(24 \%)$ & \\
\hline \multirow[t]{2}{*}{ Catheter size } & 4 French & $96(69 \%)$ & $19(76 \%)$ & \multirow[b]{2}{*}{$\mathrm{X}^{2}=.486, \mathrm{df}=1, \mathrm{p}=0.636$} \\
\hline & 5 French & $43(31 \%)$ & $6(24 \%)$ & \\
\hline \multirow[t]{3}{*}{ Allergy } & Yes & $24(17 \%)$ & $8(32 \%)$ & \multirow{3}{*}{$\begin{array}{l}\text { Not able to perform given } \\
\text { number of 'missing' }\end{array}$} \\
\hline & No & $112(81 \%)$ & $16(64 \%)$ & \\
\hline & Missing & $3(2 \%)$ & $1(4 \%)$ & \\
\hline Patient & Yes & $100(72 \%)$ & $20(80 \%)$ & Not able to perform given \\
\hline \multirow[t]{2}{*}{ Education } & No & $5(4 \%)$ & $0(0 \%)$ & number of 'missing' \\
\hline & Missing & $33(24 \%)$ & $5(20 \%)$ & \\
\hline Flush & Nurse & $20(14 \%)$ & $4(16 \%)$ & \multirow{6}{*}{$\begin{array}{l}\text { Not able to perform given } \\
\text { number of 'missing' }\end{array}$} \\
\hline \multirow[t]{5}{*}{ Person } & District Nurse & $26(19 \%)$ & $3(12 \%)$ & \\
\hline & Patient & $16(12 \%)$ & $7(28 \%)$ & \\
\hline & Relative & $12(9 \%)$ & $3(12 \%)$ & \\
\hline & N/A & $43(31 \%)$ & $4(16 \%)$ & \\
\hline & Missing & $21(15 \%)$ & $4(16 \%)$ & \\
\hline District & Yes & $73(52 \%)$ & $12(48 \%)$ & Not able to perform given \\
\hline Nursing & No & $37(27 \%)$ & $7(28 \%)$ & number of 'missing' \\
\hline Referral & Missing & $29(21 \%)$ & $6(24 \%)$ & \\
\hline
\end{tabular}


Given the level of missing data related to the variables analysed in Tables 8 and 9 it was not always possible to analyse whether there were statistical differences between those who did and those who did not have a complication. The frequency results in Table 9 do however suggest that there were a higher proportion of people (32\%) with an allergy in the complication group compared with the control group (at 17\%). There were also notable differences in who was responsible for flushing the PICC. Patient or relative were responsible for flushing the PICC in $40 \%$ of those with complications, compared with $21 \%$ of those without complications.

Given that the proportion of missing data were similar for between the complication and non-complication group this result is noteworthy. Other observable differences were that having a PICC line flushed was not a requirement for a larger percentage (31\%) of the non-complication group compared with the complication group (16\%). Once again this was not able to be analysed statistically because of the amount of missing data. 
Table 10. Summary of complications encountered, treatment and PICC removal

\begin{tabular}{|c|c|c|c|}
\hline $\begin{array}{l}\text { No of } \\
\text { patients }\end{array}$ & $\begin{array}{l}\text { Type of } \\
\text { Complication }\end{array}$ & Diagnosis and/or Treatment & $\begin{array}{l}\text { PICC } \\
\text { removed/ } \\
\text { not } \\
\text { removed } \\
\end{array}$ \\
\hline 4 & Thrombosis & Anticoagulants & $\begin{array}{l}\text { Removed }=4 \\
\text { catheters }\end{array}$ \\
\hline 4 & PICC migration & $\begin{array}{l}\text { - } \text { Migrated out to axillary vein } \\
\text { - X-ray confirmation } \\
\text { - External PICC length moved } \\
\text { from } 11 \mathrm{~cm} \text { to } 16 \mathrm{~cm} \\
\text { - } \text { Migrated out } 10 \mathrm{cms} \\
\end{array}$ & $\begin{array}{l}\text { Removed = } \\
4 \text { catheters }\end{array}$ \\
\hline 1 & PICC migration & $\begin{array}{l}\text { PICC had migrated in a short } \\
\text { distance, pulled out to correct } \\
\text { length }\end{array}$ & $\begin{array}{l}\text { Not } \\
\text { removed }\end{array}$ \\
\hline 2 & Difficulty flushing & $\begin{array}{l}\text { Urokinase instilled into PICC- } \\
\text { successfully flushed }\end{array}$ & $\begin{array}{l}\text { Not } \\
\text { removed }\end{array}$ \\
\hline 2 & Phlebitis & Conservative management- heat & $\begin{array}{l}\text { Not } \\
\text { removed }\end{array}$ \\
\hline 2 & Infection/sepsis & $\begin{array}{l}3 \text { days later Thrombosis } \\
\text { diagnosed } \\
\text { +ve PICC blood cultures but } \\
\text { PICC tip showed no growth }\end{array}$ & $\begin{array}{l}\text { Removed = } \\
2 \text { catheters }\end{array}$ \\
\hline 1 & Infection/sepsis & Antibiotics & $\begin{array}{l}\text { Not } \\
\text { removed }\end{array}$ \\
\hline 1 & Exit-site infection & MRSA +ve swab growth & Removed \\
\hline 1 & Infection/Sepsis & IV antibiotics & Removed \\
\hline 1 & Infection/Sepsis & +ve PICC cultures (coliforms) & $\begin{array}{l}\text { Not } \\
\text { removed }\end{array}$ \\
\hline 1 & $\begin{array}{l}\text { PICC exit-site looked } \\
\text { infected }\end{array}$ & No growth from swab of site & $\begin{array}{l}\text { Not } \\
\text { removed }\end{array}$ \\
\hline 1 & PICC occlusion & Could not be unblocked & Removed \\
\hline 1 & Accidental removal & $\mathrm{N} / \mathrm{A}$ & $\begin{array}{l}\text { Already } \\
\text { removed } \\
\text { (counted as } \\
\text { removed) }\end{array}$ \\
\hline 1 & Severe skin reaction & Wound dressing management & Removed \\
\hline 1 & $\begin{array}{l}\text { Minor skin reaction } \\
\text { followed by major skin } \\
\text { reaction }\end{array}$ & $\begin{array}{l}\text { Changed dressing to gauze. } \\
\text { PICC due out next day anyway. } \\
\text { Pulled one day early }\end{array}$ & $\begin{array}{l}\text { Not } \\
\text { removed }\end{array}$ \\
\hline 1 & $\begin{array}{l}\text { Widespread allergic } \\
\text { reaction to dressing and } \\
\text { cleaning solution }\end{array}$ & Wound dressing management & Removed \\
\hline
\end{tabular}

- Denotes a separate PICC pathway 
Figures 2 describes the temporal component of all PICC complications over weeks in situ. It shows that four complications occurred within the first four weeks.

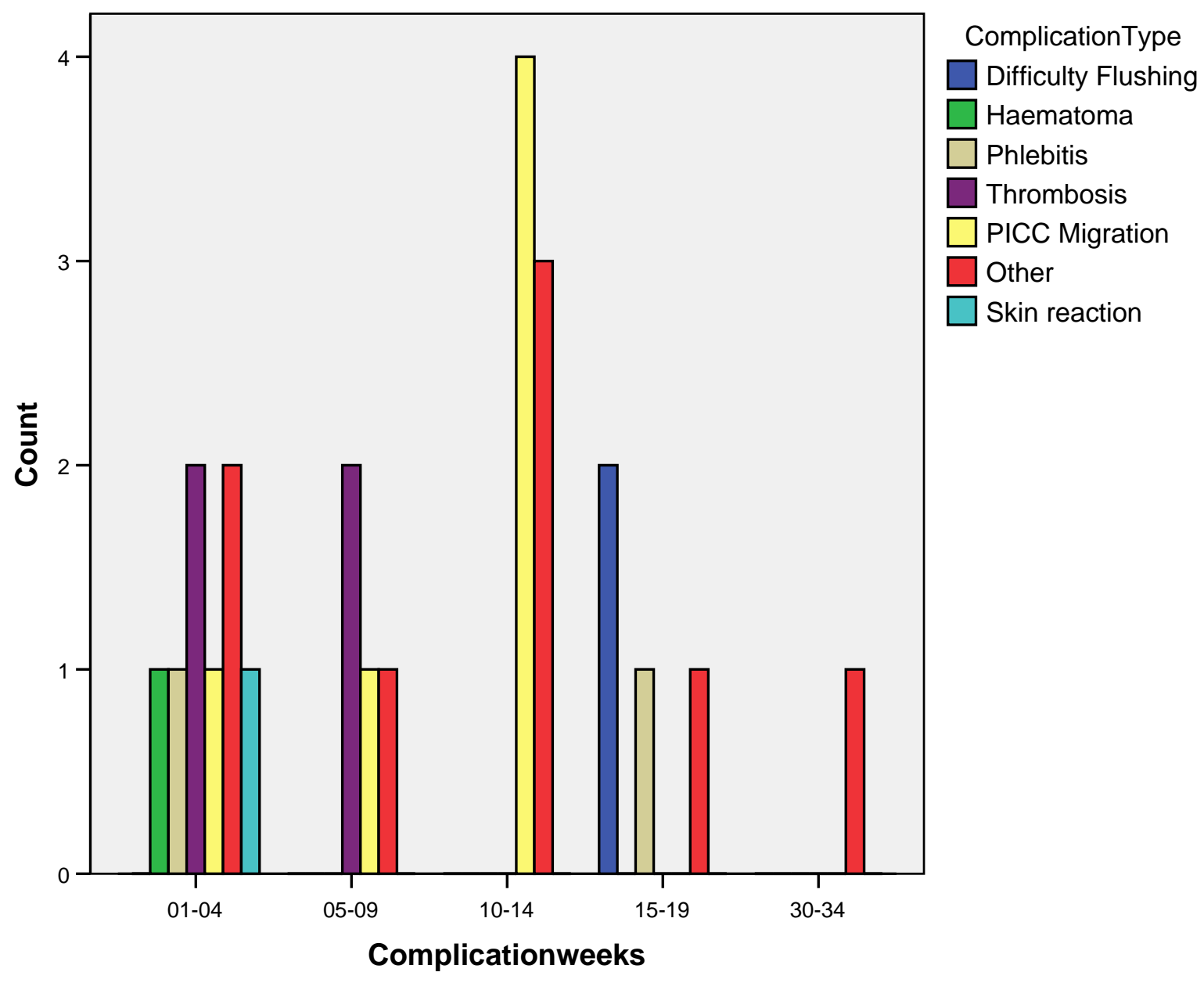

Figure 2. All PICC complications compared over time

Figure 3 shows the temporal component of all PICC complications requiring premature removal over weeks in situ. It shows that three PICCs required premature removal within the first four weeks of insertion. Importantly, only two PICCs required premature removal within the first seven days of insertion. 


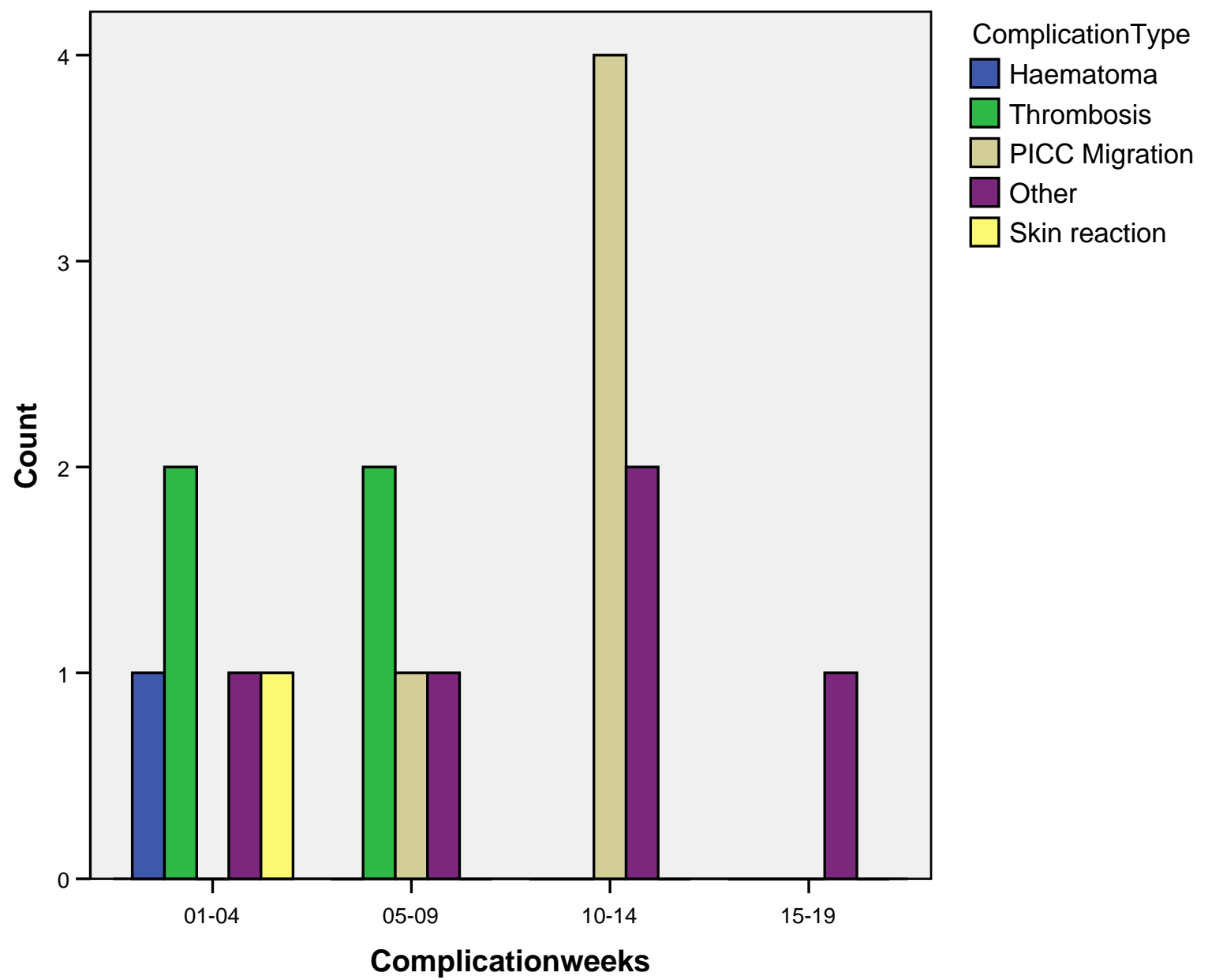

Figure 3. Complications requiring PICC removal compared over time (in weeks)

\section{Summary}

This 13-month observational study utilising a retrospective design has reported on the type, rate and incidence of polyurethane PICC-related complications in a cohort of oncology patients at Christchurch Hospital. A favourably low incidence of complications requiring premature removal was reported when compared with other similar international studies reporting on oncology cohorts. The clinical, service and research implications of these findings and their relevance to nursing practice are discussed in the following chapter, Chapter 6. 


\section{Chapter Six: Discussion}

The focus of this retrospective observational research project was to ascertain the rates and incidence of peripherally-inserted central catheter (PICC) complications within an oncology cohort over a 13-month period. The study's objectives were; to identify the complications of PICC lines, to ascertain the rates of those complications, to compare the findings with other studies, to inform practice of potential complications and, to formulate a study that had the potentiality for replication. It was intended that understanding more about the nature and rates of complications would enable the service to look at how potential complications might be minimised or ideally, prevented.

Following an extensive search of the literature (Chapter 2) it became evident that there were few research studies focusing specifically on PICC lines within oncology cohorts. Most studies described and reported on silicone (Groshong ${ }^{\mathrm{TM}}$ ) PICCs in wider populations, and yet polyurethane $\left(\right.$ Arrow $\left.^{\mathrm{TM}}\right)$ PICCs were the choice of PICC at Christchurch Hospital. Hence this study's findings and recommendations centre on polyurethane (Arrow ${ }^{\mathrm{TM}}$ ) PICC insertions in an adult oncology cohort. In this chapter the findings of this project will be compared and contrasted to the key findings in the literature. Furthermore, the findings of this research are discussed in relation to the study's objectives. It addresses the study's limitations and presents recommendations surrounding PICC use within the oncology setting.

\section{Demographics}

The current study results reported similar demographics to those of other published studies. Of the studies that reported gender, all studies showed their cohort as having a slight predominance of males over females, and that the majority was older than 50 years. This study also had more males (56\%) and had a slightly older population with a mean age of 62 years compared with 56.3 years in the Walshe et al. (2002) study and a median age of 58 years in the Yap et al. (2006) study. Cheong et al. (2004) did not report on age or gender.

There was an overwhelming predominance in the current study's cohort of gastrointestinal/colorectal cancers $(84 \%$ or $130 / 156)$. These patients are frequently 
offered infusional chemotherapies as part of their treatment, requiring PICC insertion. Yap et al. (2006) reported 67\% of their cohort as having a gastrointestinal cancer. Walshe et al. reported $23 \%$ of their group as having gastrointestinal/colorectal, however overall it was their largest group. Furthermore, as seen in most other oncology studies (except Mahalm et al.'s [2003] general hospital cohort), the current study is predominantly about patients with solid tumours.

Chemotherapy was the primary reason for PICC insertion in the present study and this was reflected in the Yap et al. (2006) and Cheong et al. (2004) studies. However, it is dissimilar to the Walshe et al. (2002) study for which only $6.3 \%$ of patients had PICC insertion for chemotherapy, with the majority of patients having PICC insertion for IV antibiotics, pain management, total parenteral nutrition, hydration, or IV access. Walshe et al. differed in that their study was based at the Memorial SloanKettering Cancer Center which cares for oncology patients from diagnosis through both their surgical and medical treatments. This means that acute surgical patients are included in their cohort. The current study's cohort was positioned in an oncology department within a general hospital as opposed to a specialised cancer centre, thus no surgical patients were included. Mahalm et al.'s study (2003) was of a general hospital cohort.

\section{Findings}

The results of this study showed four main complications that occurred within a cohort of adult oncology patients at Christchurch Hospital. These were; infection, thrombosis, PICC migration and mechanical failure, infection being the most common. Infection, thrombosis and mechanical failure all featured strongly in wider literature (Chapter 2). PICC migration was a finding unique to this study. When compared to other studies, this study showed a favourably low complication rate in all four complications. Infective complications strongly emerged in these four studies as the most frequently occurring complication. This was followed by thrombosis in Cheong et al. (2002) and Yap et al. (2006), and by phlebitis in the Walshe et al. (2002) and Mahalm et al. (2003) studies. This present study showed a pleasingly low 
rate of premature PICC removals due to complications. These findings will now be discussed.

\section{Similarities and differences amongst key studies}

In Chapter 2 similarities and differences amongst key studies surrounding PICC complications in oncology patients were outlined and discussed. Four main studies emerged as pivotal (Cheong et al., 2004; Mahalm et al., 2003; Walshe et al., 2002; Yap et al., 2006), and informed this study's design. One of the aims of this current study was to position the resultant findings within the wider body of knowledge on PICC complications in adult oncology patients. This aim proved more challenging than initially was thought. The key difference with the current study compared with the literature, was catheter composition. As well as this, each study had slightly differing cohorts, some studies had adults only, others included paediatrics. In some studies, nurses inserted the PICCs, in others, radiologists had this role. Some PICCs were inserted at the bedside, others in a sterile environment, and so on. Furthermore, within these key studies, there were differences noted in how findings were reported. Percentiles and quartiles differed slightly depending on the data distribution.

However, early on in the research process, the researcher chose to express the complication rates in this study as per 1000 catheter days, as Yap et al. suggests and O'Grady et al. (2002a) recommend. This decision was intended to enable a more precise comparison of the findings, and thus strengthen the study. This has proved beneficial and makes some valid comparisons possible.

Overall the complication rate in this study was low compared to the four key studies. The complication rate was 1.75 per 1000 catheter days, or $15 \%$ of the cohort. PICC complications requiring premature removal was even lower, at 1.12 complications per 1000 PICC days or $9.75 \%$ of the cohort (16/164, noting that all 164 PICCs were included in the analysis). This is a pleasing finding and possibilities as to why this might be the case are discussed presently. Meanwhile, the following table outlines the main similarities and differences between the four key studies and this study's findings. 
Table 11: Comparison of findings with 4 key studies

\begin{tabular}{|c|c|c|c|c|c|c|c|}
\hline Study & $\begin{array}{c}\text { No. of } \\
\text { patients }\end{array}$ & $\begin{array}{l}\text { No. of } \\
\text { PICCs }\end{array}$ & Infection & Thrombosis & $\begin{array}{c}\text { Mechanical } \\
\text { failure }\end{array}$ & $\begin{array}{c}\text { Removal due } \\
\text { to } \\
\text { complications }\end{array}$ & $\begin{array}{c}\text { Name of } \\
\text { PICC }\end{array}$ \\
\hline $\begin{array}{l}\text { Walshe et al. } \\
(2002)\end{array}$ & $\begin{array}{l}335 \\
\text { (includes } \\
\text { adults and } \\
\text { children) }\end{array}$ & 351 & $\begin{array}{l}7.4 \% \\
2.46 / 1000 \\
\text { catheter } \\
\text { days }\end{array}$ & $\begin{array}{r}3.4 \% \\
1.14 / 1000 \\
\text { catheter days }\end{array}$ & $\begin{array}{r}15.4 \% \\
5.12 / 1000 \\
\text { catheter days }\end{array}$ & $32.8 \%$ & $\begin{array}{l}\text { Silicone } \\
\text { Groshong }\end{array}$ \\
\hline $\begin{array}{l}\text { Cheong et al. } \\
\text { (2004) }\end{array}$ & 17 & 27 & $\begin{array}{l}25.7 \% \\
8 / 1000 \\
\text { catheter } \\
\text { days }\end{array}$ & $4 \%$ & $11 \%$ & $40.7 \%$ & $\begin{array}{l}\text { Silicone } \\
\text { Groshong }\end{array}$ \\
\hline $\begin{array}{l}\text { Yap et al. } \\
(2006)\end{array}$ & 73 & 88 & $\begin{array}{l}5.7 \% \\
0.73 / 1000 \\
\text { catheter } \\
\text { days }\end{array}$ & $\begin{array}{r}4.5 \% \\
0 . .58 / 1000 \\
\text { catheter days }\end{array}$ & $3.4 \%$ & $\begin{array}{r}15.9 \% \\
2.0 / 1000 \\
\text { catheter days }\end{array}$ & $\begin{array}{l}\text { Silicone } \\
\text { GroshongTM }\end{array}$ \\
\hline $\begin{array}{l}\text { Mahalm et } \\
\text { al. (2003) } \\
\text { unpublished }\end{array}$ & $\begin{array}{l}660 \\
\text { General } \\
\text { hospital } \\
\text { population }\end{array}$ & $\begin{array}{l}541 \\
\text { with } \\
\text { PICC, } \\
119 \\
\text { with } \\
\text { Midline }\end{array}$ & $\begin{array}{l}5 \% \\
\text { PICCs \& } \\
\text { Midlines } \\
\text { combined }\end{array}$ & $\begin{array}{l}\quad 1.36 \% \\
\text { PICCs \& } \\
\text { Midlines } \\
\text { combined }\end{array}$ & $\begin{array}{l}12.12 \% \\
\text { PICCs \& } \\
\text { Midlines } \\
\text { combined }\end{array}$ & $23.6 \%$ & $\begin{array}{l}\text { Polyurethane } \\
\text { Biovue }^{\mathrm{TM}} \\
\text { (withdrawn } \\
\text { half way } \\
\text { through) } \\
\text { Polyurethane } \\
\text { Arrow }\end{array}$ \\
\hline $\begin{array}{l}\text { Fairhall } \\
(2008) \\
\text { current study }\end{array}$ & $\begin{array}{l}156 \\
\text { Adult } \\
\text { oncology }\end{array}$ & 164 & $\begin{array}{l}4.3 \% \\
0.49 / 1000 \\
\text { catheter } \\
\text { days }\end{array}$ & $\begin{array}{r}2.4 \% \\
0.28 / 1000 \\
\text { catheter days }\end{array}$ & $\begin{array}{r}4.2 \% \\
1.69 / 1000 \\
\text { catheter days }\end{array}$ & $\begin{array}{r}9.75 \% \\
1.12 / 1000 \\
\text { catheter days }\end{array}$ & $\begin{array}{l}\text { Polyurethane } \\
\text { Arrow }^{\mathrm{TM}}\end{array}$ \\
\hline
\end{tabular}

\section{Infective complications}

In the present study infective complications occurred at a low rate of 0.49 per 1000 PICC days (4.3\% of cohort) compared with Walshe et al. (2002) who reported 2.46 infective complications (7.4\%) per 1000 catheter days, and Yap et al. (2006) who reported an infective complication rate of $5.7 \%$ or 0.73 complications per 1000 catheter days. Furthermore, Cheong et al. (2004) reported an infective complication rate of $25.9 \%$ at a rate of eight per 1000 PICC days. There is no one likely reason for the infections, each occurring in similar oncology cohorts but with different microorganisms, when microorganisms were known. The population, by the nature of often being on chemotherapy is at risk of infection (Rickard, 2003). 


\section{Thrombosis formation}

This study found a thrombosis complication rate of $2.4 \%$ which equates to 0.28 complications per 1000 PICC days. In comparison Walshe et al. (2002) had a thrombosis complication rate of $3.4 \%$ which equates to 1.14 complications per 1000 PICC days and Yap et al. (2006) had a thrombosis rate of $4.5 \%$ equating to 0.58 per 1000 catheter days. In terms of thrombosis prevention, earlier studies (see Chapter 2) suggested that low dose anticoagulation may decrease thrombosis formation as a PICC complication. More recent studies in oncology patients however, have not produced significant differences in outcome when low dose anticoagulation has been used. For that reason, low dose anticoagulation is not standard practice for PICC use in oncology patients at Christchurch Hospital. The low rate of thrombosis complications in this study, especially when compared to other studies, appears to support the decision not to use low dose warfarin prophylactically within this service.

\section{Mechanical failure}

The other studies report complications of leakage and breakage of the PIC catheter. For example, Walshe et al. (2002) reported 21 leaking or broken silicone catheters at a rate of 1.99 per 1000 catheter days. Mahalm et al. (2003) initially had problems with leaking or broken polyurethane catheters until the problematic catheter was withdrawn from the market. Since changing to the polyurethane (Arrow ${ }^{\mathrm{TM}}$ ) PICC, leaking and broken catheters no longer seem to feature in these statistics. This current study reports no leakage or breakage of PICCs inserted over this period. It appears that this further supports the notion that polyurethane PICCs may be more durable and flexible than silicone PICCs (Renner, 1998).

\section{PICC migration}

The present study found that PICC migration was the second most common complication. PICC migration was not mentioned in the other studies as a potential or actual complication. The Walshe et al. (2002), Cheong et al. (2002) and Mahalm et al. (2003) studies all cited mechanical failure as the third most common complication. It is important to note that this study reported five (3\%) patients with the complication of PICC migration which equates to a rate of 0.35 per 1000 catheter days. In contrast, none of the other three international studies reported PICC 
migration as a problem. They did however discuss 'accidental dislodgement' (Yap et al., 2006) on two occasions and 'accidental removal' (Walshe et al., 2002) on 19 occasions which may just be definitions of the same thing. Mahalm et al. (2003) in their local study used the same term as this study, PICC migration.

Although PICC migration in this present study was low, the question needs to be asked why this occurred at all. It is possible that the nurse didn't secure the steristrips, statlock and dressing strongly enough, or the patient may have inadvertently overused the PICC arm, or suddenly increased their intra-thoracic pressure by vomiting or coughing, or excessive diaphoresis in some patients may have led to the semi-permeable dressing, statlock dressing and steristrips lifting. Another possible explanation for PICC migration might be that the external length of the catheter is long when the patient is small in stature and frame. If the external length of the catheter is long it should be curled in a spiral around the upper arm insertion site and secured with steristrips, statlock and a semi-permeable transparent dressing. Thus, because the spiral is large it is potentially more difficult to secure with the steristrips and dressing. It is not considered best practice to trim the catheter (personal communication, Doryan Mahalm, Charge Nurse Manager, Radiology, $22^{\text {nd }}$ February, 2008).

Perhaps though, trimming the catheter could be opted for in particularly small adult oncology patients, as the PICC is routinely trimmed for paediatric patients. The reason it is not trimmed for adults and only trimmed for paediatric patients is that the PICC must be cut absolutely straight, otherwise fibrin and platelets aggregate along the jagged edge, causing catheter occlusion. In reviewing PICC management at Christchurch Hospital, we need to be mindful of the potential for PICC migration because if it migrates out a significant distance, the PICC will need replacement. It is therefore important at the formal patient education session prior to PICC insertion that there is adequate education emphasising that over-use of the PICC arm may pose the risk of PICC migration.

Currently in the setting where this research took place, IV $3000^{\mathrm{TM}}$ semi-permeable transparent dressings are used to secure the PICC, as this dressing has increased adherence properties at the outer vertical edges which should help minimise the risk 
of PICC migration. For some patients however, this area of increased adherence is where a skin reaction most often occurs. Perhaps then, when using the IV $3000^{\mathrm{TM}}$ dressing a skin protectant should be routinely used, for example Cavilon ${ }^{\mathrm{TM}}$. It could be argued that although this protectant may be expensive, if it prevented skin irritation and breakdown, the IV $3000^{\mathrm{TM}}$ dressing could be maintained without skin reaction, thus reducing cost in nurses' time and patient discomfort, or even subsequent removal of the PICC due to a major skin reaction. A prospective study could actively monitor the efficacy of this intervention.

If this solution was unworkable, then a second option might be to change from IV $3000^{\mathrm{TM}}$ dressings to a Bioclusive ${ }^{\mathrm{TM}}$ transparent semi-permeable dressings as the primary dressing. It seems that anecdotally fewer patients react to this dressing. The downside of this dressing is that it does not have the extra adherent qualities of the IV $3000^{\mathrm{TM}}$. Any change in the practice surrounding PICC dressings needs to be borne out by evidence, and here again, a prospective study has the potential to achieve this.

\section{Early PICC removal}

The complication rates in the present study that precipitated early PICC removal are also considerably lower than those found by Cheong et al. (2004) in their small retrospective study involving only 27 solid tumour oncology patients. They reported an overall complication rate of $40.7 \%$ requiring early PICC removal. They did not report on rate of complications per 1000 catheter days. To their credit a repeat prospective study two years later at the same hospital, following improvements in insertion techniques, reported a complication rate of $15.9 \%$, indicating a marked reduction in the numbers of complications (Yap et al., 2006). This second study reported that the complication rate equated to 2.0 complications per 1000 PICC days, which is a slightly higher rate, than that found in the current study. Yap et al. attribute the reduction in complications to the improvements in PICC insertion and management in their service. These included education and training of nurses to maintain the catheters, maintenance of maximum sterile barriers and caution in insertion, introduction of $2 \%$ chlorhexidine solution for antisepsis and, employment of a nurse dedicated to PICC line management and staff education. 
In the Walshe et al. study (2002) a high $32.8 \%$ of PICCs were removed due to complications, whereas in this study only $10.2 \%$ were removed for this reason (or 16/157, bearing in mind that seven PICCs which were still in situ at study's end were excluded from this analysis). In the Yap et al. study (2006) $72.7 \%$ of PICCs were removed due to completion of therapy and $15.9 \%$ removed due to complications. In this current study if completion of therapy and disease progression were combined, $77.7 \%$ of patients would have had their PICC removed due to completion of their therapy. This current study therefore has slightly better outcomes compared with the Yap et al. study.

\section{Silicone (Groshong ${ }^{\mathrm{TM}}$ ) versus polyurethane (Arrow ${ }^{\mathrm{TM}}$ )}

The Walshe et al. study examined silicone (Groshong ${ }^{\mathrm{TM}}$ ) catheters. Their population differed from those in this study in that they included paediatric patients, $3.4 \%$ of their cohort had haematological cancers, and interestingly $6.3 \%$ had no cancer. Following Walshe et al.'s lead, it was initially intended that this study would include paediatric oncology patients, however this proved too difficult to achieve due to the paediatric oncology patients being treated in a completely different department. The present study did not include patients with haematological cancers, patients who did not have cancer, or children.

The Mahalm et al. study took place in the same hospital as this present study and initially involved an examination of complications in Biovue ${ }^{\mathrm{TM}}$ PICC catheters which were withdrawn from the market in 2003 due to widespread problems with mechanical failure. Since changing to the polyurethane (Arrow ${ }^{\mathrm{TM}}$ ) PICC, it would appear that the incidence of mechanical failure has reduced markedly as demonstrated in the current study. Other major complications emerged in differing orders and included exit-site cellulitis, catheter blockage, accidental removal or dislodgement (Cheong et al, 2004; Mahalm et al, 2003; Walshe et al, 2002), major skin reaction to dressing and difficulty flushing the PICC.

The most poignant difference between the key studies and the current study was the use of the polyurethane (Arrow ${ }^{\mathrm{TM}}$ ) PICC at Christchurch Hospital. All other 
published studies used the silicone (Groshong ${ }^{\text {TM }}$ ) PICC and Mahalm et al.'s (2003) unpublished study initially used the Biovue ${ }^{\mathrm{TM}}$ polyurethane PICC and then changed to the Arrow ${ }^{\mathrm{TM}}$ polyurethane PICC at a later point. This major difference of PICC choice alone sets this present study apart from the wider literature. A secondary difference is that this study's cohort was specifically an adult oncology cohort. This specificity also sets this piece of research apart from others and thus has the potential to provide a benchmark for future studies.

\section{Why is the complication rate low?}

There are several potential contributing factors to the low complication rate reported in this study. First, patients who have PICCs in situ for short and predetermined periods of time, for example patients with colorectal cancer who have infusional fluorouracil (5-FU) chemotherapy prior to surgery, may be at a reduced risk of developing complications. This is likely to be due to these patients being newly diagnosed and relatively well going into their chemotherapy treatment, thus leading to apparently lower complication rates. These patients made up a large group within this cohort. Expressing PICC complication rates as complications per 1000 PICC days, as suggested by Yap et al. (2006) and recommended by O'Grady et al. (2002a) compensates for this and prevents over-representation of this group.

Secondly, the service at Christchurch Hospital follows evidence-based practice guidelines for insertion (as outlined in internationally recognised guidelines by O'Grady et al., 2002). Following these guidelines helps to ensure that complications are minimised. Since it is known that infection rates occurring within the first seven days post insertion are likely to be related to insertion techniques and protocols, the current study's findings of only two complications within the first seven days, suggests insertion techniques seem to be sound. Perhaps it is that PICCs are inserted in the sterile environment of the interventional suite of the Radiology Department, rather than at the bedside. It may also be because nurses inserting the PICCs wear powderless, sterile gloves, which seem to reduce the incidence of post-insertion phlebitis (see Chapter 2). 
It is possible that phlebitis may not have featured so prominently in this study's complication rate because, since 2005 it has been common practice to use ultrasound guidance at insertion. Ultrasound allows access to deeper, larger veins in the arm, thus reducing the potential for friction to develop between the PICC and the vessel wall. Previous to this, as pointed out in Chapter 3, PICCs at Christchurch Hospital were inserted into the antecubital fossa of the right arm. This seemed to be the easiest insertion point. This change has appeared to make the device easier to hide and less vulnerable to phlebitis and damage by overuse of the arm. Since usual use of the arm is important to minimise the risk of thrombus formation, this change in entry point is seen to be advantageous in terms of risk and complication minimisation.

Anecdotally, this positioning is thought to have significantly reduced the risk of mechanical phlebitis and complications overall. Furthermore, removing the effect of flexing the elbow on the catheter integrity could have had a profound effect on both mechanical failure rates and thrombosis. This change in practice may have been a key factor in keeping complications low.

To further minimise complications in PICCs, regular and ongoing education of patients and staff to follow hospital protocols, close monitoring whilst the PICC is in place, as well as early intervention of any PICC complications are paramount to reducing the risk of the PICC needing to be prematurely removed. Perhaps most importantly of all, Christchurch Hospital uses polyurethane PICCs rather than silicone. The literature indicates that there are fewer complications with polyurethane PICCs (see Chapter 2), and the current study appears to reiterate this.

Learning curve influences may have improved PICC outcomes. The team of nurses who insert PICCs is currently a very stable workforce, and this small group of nurses is likely to have developed considerable expertise over time. The oncology and district nursing workforces do not currently comprise the same stable workforce hence regular staff education is essential. Lastly, it is important to be mindful that the low complication rate may have occurred from a combination of the factors mentioned above, or from only one or even another unmentioned contributing factor. Because this was an observational study, it is not possible to describe causes and effects with this PICC data, but rather to describe and explore trends and potential relationships. This exploration is very valuable but it is important to consider that 
any conclusions about cause and effect can only be speculative because of confounding variables.

\section{Issues that arose from the findings}

In addition to the main findings reported above, the research also raised a series of issues that merit discussion. These are; known allergy, administration of antibiotics at insertion, and incomplete documentation.

\section{Known allergy}

Although not statistically significant the finding that the proportion of people with a known allergy who developed a complication (32\%) was almost double that of those who did not (at 17\%) highlights the importance of knowing about allergies as it would appear that those with allergies may be at increased risk. The missing data (three without complications and one with complications) in this variable means some caution is needed with this interpretation. It is important to ask questions regarding allergies prior to insertion to alert the inserter to potential allergies to catheters or polyurethane in particular, or specific allergies to dressings. Future research on PICCs should include allergy as a variable that is routinely collected.

\section{Administration of antibiotics at insertion}

This variable was included as previous studies had considered it may influence the possibility of infective complications. Antibiotics at insertion are strongly discouraged as per the international guidelines set out by O'Grady et al. (2002c). For this reason, no patients had antibiotics at insertion at Christchurch Hospital. Furthermore, Digital Subtraction Angiography records indicated that no patients were on continuous antibiotics at time of PICC insertion.

\section{Incomplete documentation}

In studying whether weekly dressing documentation was complete or not it was found that the records were incomplete in 139 (85\%) records. This very high percentage of missing data does not however particularly disadvantage the study's results related to complications. For the majority there was some documentation but this was sporadic, and thus an area that appeared to be poorly addressed. The reason for this is that all patients having chemotherapy through the Outpatients' Oncology 
Department had their weekly dressing changes documented on the Maintenance and Care Plan form (Appendix 6), if their dressing was changed in the department. However, if the dressing was changed by the district nurse in the patient's own home, this weekly documentation was recorded in the district nurse's file which resided in the patient's home. It can be postulated that nothing problematic was missed, because any PICC complications found by the district nurse led to the patient re-entering the Oncology Department for nursing assessment and management. Thus, even though information regarding the weekly dressings may not have been accessible to the researcher, it can be inferred that as complications were referred back to the hospital for further assessment and treatment, that this complication data were in fact complete.

Another reason for missing weekly documentation was if the patient was admitted to the oncology ward. If the PICC dressing was done on the ward, this was documented in the patient's clinical notes and not on the Maintenance and Care Plan (Appendix 6). Any complications with the PICC line during hospitalisation on the oncology ward, were to be faxed to the Oncology Outpatients' Department nurse for filing, thus ensuring data about complications were not missed.

Although the missing data did not particularly disadvantage the study results related to complications, it did however require the researcher to spend considerable time searching through clinical records for the additional information. Furthermore, it may highlight a need for a more effective system of follow-up documentation.

\section{Significant and non-significant findings}

One of the statistically significant findings in this study was the differences in patient groups between those who developed complications and those who did not. Patients within the colorectal cancer group were less likely to develop PICC complications than patients within other cancer groups. One explanation for this is that the majority of the colorectal cancer group was patients who were newly diagnosed, offered infusional fluorouracil (5-FU) chemotherapy for a short and predetermined time (about 45 days) prior to surgery, and quite well in comparison to other cancer groups who may have been being offered second or third line chemotherapy regimens. Many 
of the patients in the other cancer groups were therefore significantly sicker at the time of PICC insertion due to their disease progression or previous chemotherapy regimens.

Another notable group difference was that patients within the gastrointestinal cancer group were more likely to develop PICC complications as were those with ovarian cancer. One possible explanation for this finding is that many patients with gastrointestinal cancers are offered an intensive chemotherapy regimen namely; ECF (epirubicin, cisplatin, \& fluorouracil) or they can be offered chemotherapy with concurrent radiotherapy to the affected area. This group of patients can become severely myelosuppressed resulting in an increasing risk of infection. It therefore makes sense that this patient group might have increased risk of PICC complications. It is important to be mindful however that the overall complication rate in the current study is relatively low and thus indicates successful care of the PICC despite their compromised state.

All patients with ovarian cancer within the current study's cohort developed PICC complications. This is a puzzling finding and hasn't emerged within the wider literature. It should be noted however, that there were only three patients and five PICCs involved. Other researchers are advised to be mindful of this particular cancer group when undertaking similar studies to observe whether complications within this group are in fact more frequent.

There was no statistically significant difference in outcome between those patients who lived within the Canterbury region to those that did not, or those who had a single lumen (size 4-French) PICC versus those who had a double lumen (size 5French) PICC. Other published studies (Chapter 2) reported an increase in thrombosis complications in PICCs with lumens of larger diameter and those PICCs with multiple lumens. Other studies also reported an increase in infective complications in PICCs which received multiple catheter manipulations. This was also not borne out in this study. There appeared to be a difference in outcome (complications) when considering persons trained to be the primary person (nurse, patient, relative, and district nurse) to flush the PICC. However, the level of missing data meant that it was not possible to test for statistical significance; this also means 
one has to be cautious with the interpretation. Of the complete data, patients and families were responsible for flushing the PICC in $40 \%$ of the complication group compared with $21 \%$ of the non-complication group. More emphasis in the patient and family education sessions may need to be provided in how to prevent complications or how to recognise potential complications.

\section{Patient education}

This missing data problem also applied to whether the patient received formal education prior to PICC insertion. In this research a quarter of patients had missing data on whether they had formal education or not, making statistical testing problematic. This may not mean that education was not given, but rather that it was not given formally prior to PICC insertion, or was not documented.

Of the 156 individual patients 120 had formal education at least once. Those who did not have formal education may have included patients who had had a PICC previously and therefore did not require formal education again. Overall, it is best practice for these formal education sessions prior to PICC insertion to occur but, this is not always possible in practice. For example, sometimes patients present very unwell with their cancer, requiring immediate PICC insertion and urgent chemotherapy to follow. In such cases, the formal education session prior to insertion cannot be scheduled but, standard practice dictates that education happens more informally after insertion.

\section{Clarification surrounding analysis of complications}

In Chapter 5 it was explained that in two separate situations two complications were counted as one. In the first, the patient presented with infection followed by thrombosis three days later. It was decided that this would be counted as one complication and the cause would be infection. The rationale for this decision was that the literature explains that thrombosis often leads to infection (see Chapter 2) and that they are therefore closely related. Since infection normally follows thrombosis it is most likely that the thrombosis was pre-existing but that it had remained undiagnosed and asymptomatic in the patient until three days after the infection had been diagnosed. Although this decision to count both complications 
was appropriate at the time, on reflection combining them limits the opportunity to explore the relationship between them. It would be recommended that any researchers undertaking a similar study (whether retrospective or prospective) should count these complications separately in order to explore the relationship between the two.

In the second case, a minor skin reaction was reported early after PICC insertion and then 14 days later a major skin reaction occurred, necessitating early PICC removal. Once again these complications are probably linked, however the reason for counting them as one, was that minor complications were not the focus of this study. It was deemed important to note the minor skin reaction because it helped to explain the major complication more thoroughly. Since reflecting on the analysis, and notes taken during data collection it has become clear that minor skin reactions featured often enough to warrant further exploration. In hindsight the fact that these were not explored in this study is unfortunate, because they occurred often enough to warrant looking at how minor skin reactions can be better managed or preferably, prevented.

A second definitional issue to be considered is that of infection. In this study only one patient with infection as a complication, returned a positive PICC tip culture, which casts some doubt on whether the PICCs were actually infected, thus potentially over-estimating the infection rate. It also means that some PICCs may have been removed unnecessarily, which is a major concern. Mahalm et al. (2003) reported testing not only catheter tips for semi-quantitative bacterial counts, but also a portion of catheter that had been under the skin close to the exit site, and a swab of the catheter hub. The rationale for this was that it was believed not all infections result in a positive catheter tip culture. Infection was postulated to occur at other sources in the catheter system, without necessarily obtaining a positive catheter tip.

\section{Who should insert PICCs?}

Some studies describe radiologists inserting the PICCs, and other studies describe nurse-led PICC insertion, with radiology backup for difficult insertions. The Walshe et al. (2002) study described nurse and radiologist insertions, whereas the Cheong et al. (2004) and Yap et al. (2006) studies described radiologists as the only PICC 
inserters. Since cost effectiveness is an important consideration in PICC use, nurseled insertions were shown to be cost effective in Schmid's $(2000,2004)$ studies. One of the possible reasons why this present study shows a low rate of complications may also offer support for nurse-led insertions. A low complication rate can be assumed to contribute to cost-effectiveness since complications such as infection can add thousands of dollars to patient treatment. Walshe et al. (2002) and other studies describe nurses inserting PICC lines at the bedside; this is not the case at Christchurch Hospital. Nurses insert the PICCs in the more sterile environment of the radiology suite. Walshe et al. attempted to compare PICC outcomes with nurse insertions versus radiologist insertions.

In the Walshe et al. (2002) study, PICCs inserted by radiologists had a longer dwell time than nurse insertions, possibly due to a difference in the physical environments in which they were inserted. Nurses inserted PICCs at the patient's bedside whereas radiologists inserted them in the more sterile environment of interventional radiology. The current study's findings indicate that the complication rates associated with PICC lines in oncology patients can remain low with nurse-led insertions, coupled with adherence to education and training of nurses to insert and maintain the catheters, maintenance of maximum sterile barriers, caution in insertion in the more sterile environment of DSA, and use of $2 \%$ chlorhexidine solution for antisepsis. PICC insertion and management is going to keep changing as research and improved product designs are introduced into oncology settings.

\section{Study's limitations}

\section{Missing data}

Although the findings of this present study are valid and significant, there are some limitations to this research. Despite the earlier initiative to educate nursing staff to document all PICC data more thoroughly and completely, missing data were still an issue in this study. However, documentation had improved considerably in that the current study has been safely completed, whereas the previous retrospective study had to be abandoned due to the amount of missing data (Chapter 4). It is noticeable that the missing data tended to be from the oncology CVAD Record Form (Appendix 7), as opposed to the PICC Insertion Form (Appendix 3). It is probable that this may 
be because the oncology service is larger and more complex, in that patients access the service from differing entry points for example, the oncology ward, the Medical Day Unit, Oncology Outpatients Department, and general wards (if the oncology ward is full). Thus keeping the document on hand is a major challenge, as is completion of all items on the form. For this researcher this resulted in difficulties with some patient data in establishing the exact date of PICC removal.

It took several steps to try to retrieve this information for some patients which could be in three separate places, (i) oncology doctor's letters addressed to the general practitioner, (ii) the oncology notes which are kept separate from the clinical notes in the oncology department, and (iii) the clinical notes. Mostly, the removal date was able to be ascertained, and if it wasn't, for this study, the removal date was taken to be the day after the last documented review. Thus it could mostly be rectified by referring back to the clinical records, but in a few cases missing data were managed by a conservative estimate of PICC removal as described in Chapter 5 and in keeping with how Yap et al. (2006) managed this documentation shortfall.

It seems a significant challenge to suggest here that issues of documentation around the Central Venous Access Record form (Appendix 7) should be streamlined, considering the many areas where the patient can present with needing treatment or attention to their PICC. The most logical solution appears to be (i) an on-line form so that wherever the nurse is when she/he accesses the PICC she or he would be able to go into the computer and with password access bring up the patient's on-line form and enter the data. This could be filed individually in the patient's own file or in a computer folder with all oncology patients with a PICC line, and (ii) ultimately clinical notes to be full on-line versions. The result would mean wider access by all staff throughout the hospital context (who had password access) to all the information about the PICC line and its management in each individual patient.

In terms of reviewing the issues regarding the Central Venous Access Device form, the researcher has elicited what is important from an audit and clinical perspective. The form has four major problems as it is currently used. First, the form still does not get completed fully by all nurses. Secondly, there is important information missing from the form which should be included. This includes cancer type and cancer site, 
as well as evidence of metastases or not (discussed further below). This is important information for research and clinical purposes. Thirdly, there are some items included on the form which are not necessary for clinical or audit purposes. There are also prompts for circling that do not accurately reflect the complications expected. Since it is important to encourage nurses to complete the form fully, it is essential that this be rectified by updating and improving the form to make it more relevant for both clinical and audit purposes (as proposed in Appendix 8).

Fourthly, the Central Venous Access Device form is currently held in one place (the Oncology Outpatients Department) but the patients can present at many places, such as Medical Day Unit, Emergency Department, oncology ward, or another medical ward. Thus the current practice of having the form in one place but the patient potentially presenting at many places makes it a real challenge to ensure the form is fully completed. Hence the suggestion of on-line access to the form for passwordapproved health professionals within the hospital.

Sometimes the region that the patient lived in was missing if a patient label had not been applied to the form. If this study were to be replicated, having this data (sticky label, cancer type and site, metastases or not) on the form would avoid the necessity to access the patient's oncology letters, and thus minimise data collation time significantly. A proposed new form (Appendix 8) would also include a space to place the sticky label with a border around it as this has important demographic data to include.

\section{Individual variation in reporting of PICC outcomes}

The fact that patients are evaluated in a wide array of inpatient and outpatient settings by different nurses may lead to variability in interpretation and documenting of PICC outcomes. It was hoped that this potential limitation was minimised by educating nurses well about accurate documentation. Also, this would not have affected the overall complication rate because early PICC removal was a clear definitional endpoint which was a medical decision (Walshe et al., 2002). Description of complications may have been affected however. 
Another limitation was that with infection complications there may not have been consistency by the oncologists about whether the PICC was prematurely removed or not. It is possible that this did not affect the outcome of the study, in that the number of oncology patients with infection was only seven.

Because this study focused on the relatively ill cohort of cancer patients, these results may not be able to be generalised to the general hospital population. However, they can be quite safely compared to other oncology cohorts or patients with other serious medical illnesses.

The decision to define the unit of measure as the PICC placement on some occasions, and the individual patient on other occasions may have been confusing and potentially may have misrepresented certain risks or demographic features. In the end, a 'commonsense' approach was taken when defining the unit of measure, one that made sense to the researcher at the time of data analysis.

\section{Potential for observer bias}

Observational studies have the potential problem of observer bias. In the methodology chapter (Chapter 4) ways this researcher attempted to minimise observer bias were described in detail. For example, because the research design was retrospective, missing or 'messy' data were managed by going to other sources and extracting data directly from the clinical notes if there were gaps. The researcher was not 'forcing' a response or answer to questions. Nurses used prompts to circle as well as open ended answers so that any complications could be adequately described. In this way a case was laid of careful management of the data, thus minimising observer bias. A potential limitation was that the researcher had to work with the data that were there, which were data created for a different purpose to research. Because the study was retrospective, clinicians were not forewarned of the research, thus the 'real world' of PICC management could be captured. The purpose of the study however, had been to better describe what appeared to be an increase in complications within the service. In this way, the researcher could have been accused of researcher bias. However, the researcher was careful to go into the data collection process with no preconceived views of what the findings might be. Also, the data collection time had 
been adequate and the sample size was reasonable for inferring statistical significance. Only one patient was lost to follow-up, thus minimising the risk of undetected adverse outcomes.

\section{Designing future studies}

The strength of a retrospective study like this one is that the 'real world' of nursing can be observed and captured over a designated span of time with minimal observer bias. Retrospective studies are also much cheaper and quicker to complete as the data have already been collected. Recommendations that emerge from a retrospective study can inform practice and lead to change; however the evidence from a quality prospective study can be better. Because this study was retrospective rather than prospective, the researcher found some data missing. A prospective study could be more attentive to detail. To achieve this it would be helpful to have a process where there was weekly assessment of data and a system for tracking anything incomplete. It could include the documentation by district nurses in the patient's file located in their own home, as well as the oncology ward documentation. As a point to consider in preventing complications, the weight and height of the patient may be useful data to collect prospectively. Weight may influence the risk for thrombosis formation and, weight and height may influence the risk of PICC migration. These are anecdotal perceptions of relationships which may or may not exist, and require evidence as to whether this might be the case.

Prospective studies, where a research design is in place prior to beginning data collection, may lead to more flexibility and more efficiency in undertaking clinical research in that confounding variables can be minimised (Mann, 2003) and if another variable presents itself as requiring investigation, it may be added to an already existing audit tool and quickly reviewed. The main advantage of a prospective study however, is a significantly reduced risk of data omission as there would be more vigilance surrounding data collection.

In hindsight, this researcher has concluded that also reporting all minor complications would have been very valuable, in order to pick up problematic trends early. The reason that this did not occur was mainly to do with following the designs 
of previous studies. For example, one minor complication is that of minor skin reaction. Although the numbers were not routinely collected, there appeared to be a pattern of occurrence which would warrant further investigation and possible change in practice with regard to applying a skin protector first and/or changing the primary type of semi-permeable dressing.

\section{Recommendations}

This study was undertaken within the Oncology Department of Christchurch Hospital. It represents a typical cohort of adult oncology patients with a PICC insertion for cancer treatment. With the pleasing finding of a low complication rate with the polyurethane (Arrow ${ }^{\mathrm{TM}}$ ) PICC currently used in this facility, there is strong evidence to support its continued use.

As insertions are currently undertaken within the sterile environment of the Interventional Suite of the Radiology Department using ultrasound guidance coupled with use of powderless, sterile gloves, this nurse-led protocol should also continue. It is also recommended that O'Grady et al.'s (2002) internationally recognised guidelines for effective insertion and management of PICC lines also continue to be followed.

Although improvement in documentation was noted between a previous attempt to undertake a retrospective observational study within the same context, and this present study, gaps still emerged. It is recommended that there is continued effort in ensuring nurses fill in the forms fully and as accurately as possible so as to ensure an adequate audit trail. This is a challenge when the patient can present at different departments within the hospital. Perhaps an on-line form for PICC management documentation could be developed (Appendix 8). This form via password entry can then be accessed at any port of entry to the hospital by the health practitioner managing the patient with a PICC.

The findings of the current study raise important clinical practice and service issues for the institution where this research took place. Some of the limitations to the current study arose because clinical records were used for research purposes. The 
PICC insertion form was thorough and comprehensive, but the Central Venous Access Device (CVAD) form proved inadequate for research purposes. It is therefore recommended that other services who want to develop a framework for researching their own practices ensure that their own documentation around PICC insertion and management is rigorous and withstands the audit process. Auditing practice is fundamental to effectively maintaining and improving patient outcomes. Forms that record such data must be robust. To this end, Appendix 7 has been reformatted by the researcher to more adequately capture relevant data for both service and research purposes (see Appendix 8). It can therefore also be argued that the research process can illuminate shortcomings in current documentation processes, with the view to improving them. Perhaps it might be that the PICC/Midline Patient Information form (Appendix 3) meets research needs because it was developed and audited by a nurse who had researched the topic, whereas the CVAD Record form was not. The research process helps clinical practitioners to look at practice from a research perspective and include it in their global picture of practice.

As there is a reassuringly low rate of complications surrounding PICC use in this cohort of oncology patients at Christchurch Hospital, it suggests that continued comprehensive education of patients and staff in PICC management, may minimise complications. Monitoring PICC lines closely whilst in place, and ensuring early intervention surrounding PICC complications can also help keep the complication rate low. Maintaining current knowledge around protocols by reviewing relevant literature as well as responding appropriately to evidence-based recommendations and manufacturer's latest recommendations, should also contribute to maintaining a low complication rate.

It is recommended that nurses within an oncology department, whether that be within the institutional setting or in the wider outpatient community, who have specialist knowledge regarding PICCs and their management, be identified as PICC resource nurses. Depending on each facility's PICC use, a nurse may be required solely for that role. Complications can be costly to healthcare providers and the early interventions of, adequate education for patients and staff, noticing emergence of complications early, and prevention of early PICC removal, should prove to be cost effective overall for the facility and improve quality of life for oncology patients. 
Currently at Christchurch Hospital a self-directed learning package is in the process of being formulated which focuses on CVADs overall, but has a large component on PICCs. Furthermore, this package is also coupled with CVAD study days.

This study revealed a need for the Central Venous Access Device form (Appendix 7) to be updated as proposed (Appendix 8). Should forms be specifically prepared for research purposes, it is recommended that they be pre-tested to ensure items accurately reflect variables wanting to be explored in the research. Should forms be prepared for documentation and audit processes, it is recommended that these too be regularly reviewed and updated so as to adequately reflect manufacturers' recommendations, clinical needs, and research findings.

As has been discussed earlier in this chapter, a prospective observational study is highly recommended not only within Christchurch Hospital, but more widely in other oncology services. Particularly for this hospital, a prospective study closely following a retrospective study may further confirm the low complication rate findings of this present study. An area for further research would be to examine more closely the relationship between cancer diagnosis and PICC complication rates. Use of the proposed new form (Appendix 8), coupled with the more focused staff education study days and learning package, may further strengthen the place of effective education in reducing, minimising and ideally avoiding complications surrounding PICCs. It is proposed that a prospective study will significantly diminish the challenge of missing data, which hampered this present study. It is further recommended that subsequent studies include minor complications as part of data collection, as it is suspected that minor complications may be the precursor to major complications.

\section{Summary}

PICCs have become a mainstay of treatment delivery within oncology both nationally and internationally. As became evident through the literature and confirmed within this study, PICCs are likely to be cost effective, have potential for a low complication rate, are easy to insert and remove, and appear to lead to improved quality of life for oncology patients requiring intravenous treatment. The primary 
aims of the study have been achieved. The complications of PICC lines have been identified as infective, thrombotic and mechanical failure (breakage, leakage, catheter occlusion), as well as accidental removal and PICC migration. Furthermore, the rate of complications compared favourably with other studies and recommendations were included to ensure the study can be successfully replicated by others in the oncology setting. Whilst replication is a possibility it is strongly recommended that a similar study utilise a prospective research design.

The current study began out of a perceived increase in the number of PICC complications within the oncology setting of Christchurch Hospital (Chapter 1). At the time it was suggested that it might be due to either a real increase in complications or an increase in the number of PICCs being inserted within this patient population. It is pleasing to note that the research process has established that a favourably low rate of PICC complications was in fact the reality and thus the perceived increase in PICC complications may be attributed to an increase in PICC use for oncology treatment delivery rather than an increase in PICC complications per se. The literature revealed that the more the PICC is accessed the higher the complication rate. The current study has shown that with the polyurethane (Arrow ${ }^{\mathrm{TM}}$ ) catheter used at Christchurch Hospital, despite it being accessed daily when not in use, the complication rate can still be kept low. The laboratory-based literature (Chapter 2) had supported Christchurch Hospital's insertion team's view that polyurethane PICCs were safer and easier to insert, with less complications than silicone PICCs. This benchmark study reflects and supports that view and therefore contributes to the debate surrounding PICC choice. Furthermore, a subsequent study would be well positioned to compare this cohort's PICC complication rate with another researcher's cohort in the future. Suggestions for further research include examining more closely the relationship between cancer diagnosis and PICC complications. 
Appendices 
Appendix 1 - Upper-South Regional Ethics Committee Approval 
Appendix 2 - A Guide to your PICC/Midline: CLC2000-saline lock 
Appendix 3 - PICC/Midline Patient Information form 
Appendix 4 - Canterbury District Health Board Protocols for Peripherally Inserted Central Catheters Management 
Appendix 5 - De-Clotting a PIC Catheter using the 'POP Technique' 
Appendix 6 - Maintenance and Care Plan form 
Appendix 7 - Central Venous Access Device Record form 
Appendix 8 - Proposed Central Venous Access Device Record form 
Appendix 9 - Canterbury District Health Board Approval for use of its documents within this thesis 


\section{References}

Abi-Said, D. I., Raad, I. I., Umphrey, J., Gonzalez, V., Richardson, D., Marts, K. et al. (1999). Infusion therapy team and dressing changes of central venous catheters. Infection Control Hospital Epidemiology, 20(2), 101-105.

Allen, A. W., Megargell, J. L., Brown, D. B., Lynch, F. C., Singh, H., Singh, Y. et al. (2000). Venous thrombosis associated with the placement of peripherally inserted central catheters. Journal of Vascular and Interventional Radiology, 11(10), 1309-1314.

Anderson, A. J., Krasnow, S. H., Boyer, M. W., Cutler, D. J., Jones, B. D., Citron, J. L., Ortega, L. G., \& Cohen, M. H. (1989). Thrombosis: The major Hickman catheter complication in patients with solid tumour. Chest, 95, 71-75.

Angle, J. F., Matsumoto, A. H., Skalak, T. C., O'Brien, R. F., Hartwell, G. D., \& Tegtmeyer, C. J. (1997). Flow characteristics of peripherally inserted central catheters. Journal of Vascular and Interventional Radiology, 8(4), 569-576.

Archibald, L. K., \& Gaynes, R. P. (1997). Hospital-acquired infections in the United States. The importance of interhospital comparisons. Infectious Diseases Clinics North America, 11(2), 245-255.

Beanland, C., Schneider, Z., LoBiondo-Wood, G., \& Haber, J. (1999). Nursing research: Methods, critical appraisal and utilisation. Sydney, NSW: Mosby.

Bern, M. M., Lokich, J. J., Wallach, S. R., Bothe, A. Jr., Benotti, P. N., Arkin, C. F., Greco, F. A., Huberman, M., \& Moore, C. (1990). Very low doses of warfarin can prevent thrombosis in central venous catheters. A randomized prospective trial. Annals of Internal Medicine, 112, 423-428.

Boraks, P., Seale, J., Price, J., Bass, G., Ethell, M., Keeling, D. et al. (1998). Prevention of central venous catheter associated thrombosis using minidose warfarin in patients with haematological malignancies. British Journal of Haematology, 101, 483-486.

Bowe-Geddes, L. A., \& Nichols, H. A. (2005). An overview of peripherally inserted central catheters. Topics in Advanced Practice Nursing e-Journal, 5(3), 1-9.

Brink, P. J., \& Wood, M. (1994). Advanced design in nursing research ( $2^{\text {nd }}$ ed.). Boston: Sage.

Brown, J. D., Moss, H. A., \& Elliott, T. S. (1997). The potential for catheter microbial contamination from a needleless connector. Journal of Hospital Infection, 36, 181-189.

Burns, N. \& Grove, S.K. (2001). The practice of nursing research: Conduct, critique, and utilisation. ( $4^{\text {th }}$ ed.). Philadelphia: W.B. Saunders Company.

Cardella, J. F., Cardella, K., Bacci, N., Fox, P.S. \& Post, J.H. (1996). Cumulative experience with 1273 peripherally inserted central catheters at a single institution. Journal of Vascular and Interventional Radiology, 7, 5-13.

Chambers, S. T., Sanders, J., Patton, W. N., Ganly, P., Birch, M., Crump, J. A., \& Spearing, R.L. (2005). Reduction of exit-site infections of tunnelled intravascular catheters among neutropenic patients by sustained-release chlorhexidine dressing; results from a prospective randomised controlled trial. Journal of Hospital Infection, 61, 53-61.

Chemaly, R. F., de Parres, J. B., Rehm, S. J., Adal, K. A., Lisgaris, M. V., KatzScott, D. S., et al. (2002). Venous thrombosis associated with peripherally inserted central catheters: A retrospective analysis of the Cleveland Clinic experience. Journal of Clinical Infectious Diseases, 34, 1179-1183. 
Cheong, K., Perry, D., Karapetis, C., \& Koczwara, B. (2004). High rate of complications associated with peripherally inserted central venous catheters in patients with solid tumours. Internal Medicine Journal, 34, 234-238.

Chernecky, C. (2001). Satisfaction versus dissatisfaction with venous access devices in outpatient oncology: A pilot study. Oncology Nurses Forum, 28(10), 16131616.

Cook, T. D., \& Campbell, D. T. (1979). Quasi-experimentation: Design and analysis issues for field settings. Chicago: Rand McNally.

Costerton, J. W. (1995). Overview of microbial biofilms. J Ind Microbiology, 15(3), 137-140.

Crookes, P. A., \& Davies, S. (1998). Research into practice: Essential skills for reading and applying research in nursing and health care. Marrickville, NSW: Balliere Tindall.

Doll, R. (2001). Cohort studies: History of the method II. Retrospective cohort studies. Social and Preventative Medicine (International Journal of Public Health), 46, 152-160.

Donowitz, G. R., Maki, D. G., Crnich, C. J., Pappas, P. G., Rolston, K. V. (2001). Infections in the neutropenic patient: New views of an old problem. Hematology, 113-139.

Duerksen, D. R., Papineau, N., Siemens, J., \& Yaffe, C. (1999). Peripherally inserted central catheters for parenteral nutrition: a comparison with centrally inserted catheters. Journal of Parenteral and Enteral Nutrition, 23(2), 85-89.

Eastman, M. E., Khorsand, M., Maki, D. G., Williams, E. C., Kim, K., Sondel, P. M., Schiller, J. H., \& Albertini, M. R. (2001). Central venous device-related infection and thrombosis in patients treated with moderate dose continuousinfusion interleukin-2. Cancer, 91, 806-814.

Eastridge, B. J., \& Lefor, A. T. (1995). Complications of indwelling venous access devices in cancer patients. Journal of Clinical Oncology, 13(1), 233-238.

Elliott, T. S., Faroqui, M. H., Armstrong, R. F., \& Hanson, G. C. (1994). Guidelines for good practice in central venous catheterisation. Journal of Hospital Infection, 28(3), 163-176.

Farjo, L. (2003). Blood collection from peripherally inserted central catheters: an institution's effort to evaluate and update its current policy. Journal of Infusion Nursing, 26(6), 374-379.

Fetzer, S. J., \& Manning, G. P. (2004). Safety and efficacy of the POP technique for restoring patency to occluded PIC catheters. Applied Nursing Research, 17(4), 297-300.

Gillis, A., \& Jackson, W.F. (2002). Research for nurses: Methods and interpretation. Philadelphia, Pennsylvania: Davis Company.

Goodwin, M. L., \& Carlson, I. (1993). The peripherally inserted central catheter: A retrospective look at three years of insertions. Journal of Intravenous Nursing, 16(2), 92-104.

Gould, J. R., Carloss, H. W., \& Skinner, W. L. (1993). Groshong catheter-associated subclavian venous thrombosis. Am J Med, 95, 419-423.

Grove, J. R., \& Pevec, W. C. (2000). Venous thrombosis related to peripherally inserted central catheters. Journal of Interventional Radiology, 11(7), 837840.

Hamilton, H. (2006). Complications associated with venous access devices: part one. Nursing Standard, 20(26), 43-50. 
Hardy, A., \& Magnello, E. (2002). Statistical methods in epidemiology: Karl Pearson, Ronald Ross, Major Greenwood and Austin Bradford Hill, 19001945. Social and Preventative Medicine (International Journal of Public Health), 47, 80-89.

Heaton, D. C., Han, D. Y., \& Inder, A. (2002). Minidose (1mg) warfarin as prophylaxis for central vein catheter thrombosis. Internal Medicine Journal, 32, 84-88.

Hoffmann, K. K., Weber, D. J., Samsa, G. P., \& Rutala, W. A. (1992). Transparent polyurethane film as an intravenous catheter dressing. A meta-analysis of the infection risks. JAMA, 267, 2072-2076.

Horattas, M. C., Trupiano, J., Hopkins, S., Pasini, D., Martino, C., \& Murty, A. (2001). Changing concepts in long-term central venous access: Catheter selection and cost savings. American Journal of Infection Control, 29(1), 3240.

Intravenous Nurse Society. (1997). Position paper: Peripherally inserted central catheters. Journal of Intravenous Nursing, 4(20), 172-174.

Intravenous Nursing New Zealand (IVNNZ) PICC Level 1 study day handout (1995). Christchurch Hospital: Department of Nursing.

Johnstone, M.-J. (1994). Bioethics: A nursing perspective. Sydney: W.B Saunders.

Kuter, D. J. (2004). Thrombotic complications of central venous catheters in cancer patients. The Oncologist: Symptom management and supportive care 9, 207216.

Last, K. W., Mansi, J. L., Oakley, C., \& Lofts, F. J. (1998). Long term intravenous access devices: Superiority of peripherally inserted central cannulae (PICCs) over Hickman Catheters (HCs). Annals of Oncology, 9(4), 142.

Ligthelm, R. J., Borzi, V., Gumprecht, J., Kawamori, R., Wenying, Y., \& Valensi, P. (2007). Importance of observational studies in clinical practice. Clinical Therapeutics, 29, 1284-1292.

Luepker, R. V. (2005). Observational studies in clinical research. Journal of Laboratory Clinical Medicine, 146, 9-12.

Maas, M. L., Buckwalter, K. C., Reed, D., \& Pringle Specht, J. K. (1994).

Mack, D. (1999). Molecular mechanisms of staphylococcus epidermidis biofilm formation. Hosp Infect, 43, 113-125.

Macklin, D., \& Chernecky, C. (1997). How to manage PICC's. American Journal of Nursing, 97(9), 26-32.

Macklin, D., \& Chernecky, C. (2004). Real world nursing survival guide: IV therapy. St. Louis, Mo.: Saunders.

Magagnoli, M., Masci, G., Carnaghi, C., Zucali, P. A., Castagna, L., Morenghi, E., \& Santoro, A. (2003). Minidose warfarin is associated with a high incidence of international normalised ratio elevation during chemotherapy with FOLFOX regimen. Annals of Oncology, 14, 959-960.

Mahalm, D., Pithie, A., \& Chambers, S. (2003). The establishment of a peripherally inserted central catheter [PICC] service in Christchurch Hospital.

Christchurch Hospital.

Maki, D.G. (1991). Infection caused by intravascular devices: Pathogenesis, strategies for prevention. ( $1^{\text {st }}$ ed.) London: Royal Society of Medicine Services.

Maki, D.G. (1994). Yes, Virginia, aseptic technique is very important: Maximal barrier precautions during insertion to reduce the risk of central venous catheter-related bacteraemia. Infect Control Hosp Epidemiol, 15, 227-230. 
Maki, D. G., Bennett, J., \& Bractiman, P. (Eds.) ( 1992). Infections due to Infusion Therapy. ( $3^{\text {rd }}$ ed.). Boston: Little Brown \& Co.

Maki, D., \& Ringer, M. (1987). Evaluation of dressing regimens for prevention of infection with peripheral intravenous catheters. JAMA, 258(17), 2396-2403.

Maki, D. G., \& Mermel, L. A. (1998). Infections due to infusion therapy. In J. V. Bennett \& P. S. Brachman (Eds.), Hospital Infections ( $4^{\text {th }}$ ed., pp. 689-724). Philadelphia: Lippincott-Raven.

Maki, D. G., Ringer, M., \& Alvarado, C. J. (1991). Prospective randomised trail of povidone-iodine, alcohol and chlorhexidine for prevention of infection associated with central venous and arterial catheters. Lancet, 338, 339-343.

Maki, D. G., Stolz, S., Wheeler, S., \& Mermel, L. A. (1994). A prospective, randomised trial of gauze and two polyurethane dressings for site care of pulmonary artery catheters: implications for catheter management. Critical Care Medicine, 22, 1729-1937.

Mann, C. J. (2003). Observational research methods. Research design II: cohort, cross sectional, and case-control studies. Emergency Medical Journal, 20, 5460 .

Marin, M. G., Lee, J. C. \& Skurnick, J. H. (2000). Prevention of nosocomialbloodstream infections: effectiveness of antimicrobial-impregnated and heparin bonded central venous catheters. Critical Care Medicine, 9, 33323338

Masci, G., Magagnoli, M., Zucali, P.A., Castagna, L., Carnaghi, C., Sarina, B. et al . (2003). Minidose warfarin prophylaxis for catheter-associated thrombosis in cancer patients: can it be safely associated with fluorouracil-based chemotherapy? Journal of Clinical Oncology, 21, 736-739.

Masoorli, S. (1997). Managing complications of central venous access devices. Nursing, August, 59-64.

Masoorli, S., \& Angeles, T. (1990). PICC lines: The latest home care challenge. Registered Nurse, 53, 44-51.

Masoorli, S., \& Angeles, T. (2002). Getting a line on CVAD central vascular access devices. Nursing, 32(4), 36-45.

Mathers, N., \& Huang, Y. C. (1998). Evaluating methods for collecting data in published research. In P. A. Crookes \& S. Davies (Eds.), Research into practice: Essential skills for reading and applying research in nursing and health care. (pp. 139-180). Marrickville, NSW: Balliere Tindall.

Matthews, L., \& Venables, A. (1998). Critiquing ethical issues in published research. In P. Crookes \& S. Davies (Eds.), Research into practice: Essential skills for reading and applying research in nursing and health care. (pp. 204-232). Marrickville, NSW: Balliere Tindall.

Mayo, D. J., Helsabeck, C. B., \& Horne, M. K. (1995). Intraluminal clots in groshong catheters. Journal of Vascular Access Devices, 1(2), 20-22.

Mazzola, J. R., Schott-Baer, D., \& Addy, L. (1999). Clinical factors associated with the development of phlebitis after insertion of a peripherally inserted central catheter. Journal of Intravenous Nursing, 22(1), 36-42.

Mermel, L. A. (2000). Prevention of intravascular catheter-related infections. Annals of Internal Medicine, 132(5), 391-402.

Mimoz, O., Pieroni, L., Lawrence, C., Edouard, A., Costa, Y., Samii, K., et al. (1996). Prospective, randomised trial of two antiseptic solutions for prevention of central venous or arterial catheter colonisation and infection in intensive care unit patients. Critical Care Medicine, 24, 1818-1823. 
Monreal, M., Alastrue, A., Rull, M., Mira, X., Muxart, J., Rosell, R., \& Abad, A. (1996). Upper extremity deep vein thrombosis in cancer patients with venous access devices - prophylaxis with a low molecular weight heparin (Fragmin). Thromb Haemost, 75, 251-253.

Moran, A. B., \& Camp-Sorrell, D. (2002). Maintenance of venous access devices in patients with neutropenia. Clinical Journal of Oncology Nursing, 6(3), 1-5.

Ng, P., Ault, M. J., Ellrodt, A. G., \& Maldonado, L. (1997). Peripherally inserted central catheters in general medicine. Mayo Clin Proc, (72), 225-233.

Nightingale, C. E., Norman, A., Cunningham, D., Young, J., Webb, A., \& Filshie, J. (1997). A prospective analysis of 949 long-term central venous access catheters for ambulatory chemotherapy in patients with gastrointestinal malignancy. European Journal of Cancer Care, 33, 398-403.

O'Grady, N. P., Alexander, M., Patchen Dellinger, E., Gerberding, J. L., Heard, S. O., Maki, D. G., et al. (2002a). Guidelines for the Prevention of Intravascular Catheter-Related Infections. Natl Centre Infect Dis, 51(RR10), 1-26.

O'Grady, N.P., Alexander, M., Patchen Dellinger, E., Gerberding, J.L., Heard, S., Maki, D.G. et al. (2002b). Guidelines for preventing infections associated with the insertion and maintenance of central venous catheters. Journal of Hospital Infection, 47 (Supp), 47-67.

O'Grady, N.P., Alexander, M., Patchen Dellinger, E., Gerberding, J.L., Heard, S.O., Maki, D.G. et al. (2002c). Guidelines for the prevention of intravascular catheter-related infections. Paediatrics, 110(5), 476-489.

Orr, M. E., \& Ryder, M. A. (1993). Vascular access devices: perspectives on designs, complications, and management. Nutrition in Clinical Practice, 8(4), 145152.

Pallant, J. (2002). SPSS survival manual: A step by step guide to data analysis using SPSS for windows. Crows Nest, NSW: Allen \& Unwin.

Pasquale, R. D., Campbell, J. M., \& Magnant, C. M. (1992). Groshong versus hickman catheters. Obstetrics, 174, 408-410.

Peat, J. (2001). Health science research: A handbook of quantitative methods. Crows Nest, NSW: Allen and Unwin.

Pemberton, L.B., Lyman, B., Lander, V., \& Covinsky, J. (1986). Sepsis from triplevs. single-lumen catheters during total parenteral nutrition in surgical or critically ill patients. Archives of Surgery, 121, 591-594.

Pittet, D., Tarara, D., \& Wenzel, R. P. (1994). Nosocomial bloodstream infections in critically ill patients: Excess length of stay, extra costs and attributable mortality. JAMA, 271(20), 1598-1602.

Polak, J.F., Anderson, D., Hagspiel, K., \& Mungovan. (1998). Peripherally inserted central catheters: Factors affecting patient satisfaction. American Journal of Roentgenology, 170, 1609-1611.

Polit, D. F., Beck, C. T., \& Hungler, B. P. (2001). Essentials for nursing research: Methods, appraisal, and utilisation. Philadelphia: Lippincott.

Potera, C. (1999). Forging a link between biofilms and disease. Science, 283(5409), 1837-1839.

Raad, I., Costerton, W., Sacilowski, M., Annaissie, F., \& Bodey, G. P. (1993). Ultrastructural analysis of indwelling catheters; A quantitative relationship between luminal colonisation and duration of placement. Journal of Infective Diseases, 168(400-407).

Ramsden, I. (1993). Kawa Whakaruruhau - Cultural safety in nursing education in Aotearoa. Nursing Praxis in New Zealand, 8(3), 4-10. 
Ray, S., Stacey, R., Imrie, M., \& Filshie, J. (1996). A review of 560 Hickman catheter insertions. Anaesthesia, 51, 981-985.

Reichardt, P., Kretzschmar, A., Biakhov, M., Karthaus, M., Marschner, N., Kroning, H. et al. (2002). A Phase III randomised, double-blind, placebo-controlled study evaluating the efficacy and safety of daily low-molecular-weight heparin (dalteparin sodium, fragmin) in preventing catheter-related complications (CRCs) in cancer patients with central venous catheter (CVCs). Proc Am Soc Clin Oncol, 21, 369a.

Renner, C. (1998). Polyurethane vs. silicone PICC catheters: A brief review of characteristics and clinical outcomes. Journal of Vascular Access Devices, 3(1), 16-21.

Rickard, C. (2004). Central venous catheters: A survey of ICU practices. Issues and Innovations in Nursing Practice, 48(3), 247-256.

Rickard, N. (2003). Reducing infections associated with central venous catheters. British Journal of Nursing, 12(5), 274-284.

Rosovsky, R. P., \& Kuter, D. J. (2005). Catheter-related thrombosis in cancer patients: Pathophysiology, diagnosis, and management. Haematology/Oncology Clinics of North America, 19, 183-202.

Rotstein, C., Brock, L., \& Roberts, R. S. (1995). The incidence of first Hickman catheter-related infection and predictors of catheter removal in cancer patients. Infection Control Hospital Epidemiology, 16(8), 451-458.

Ryder-Lewis, M. (2004). Reliability study of the sedation-agitation scale in an intensive care unit. Unpublished Master of Arts (Applied) thesis, Victoria University of Wellington.

Sanders, J. (2006). A prospective double blind randomised clinical trial of $70 \%$ ethanol to prevent catheter related sepsis in tunnelled catheters in haematology patients treated with chemotherapy. University of Otago, Dunedin.

Schmid, M. M. (1994). A case control study on the cost differences between peripherally inserted central catheters and central venous catheters. University of Michigan, Ann Arbor.

Schmid, M. W. (2000). Risks and complications of peripherally and centrally inserted intravenous catheters. Critical Care Nursing Clinics of North America, 12(2), 165-173.

Schwarz, R. E., Coit, D. G., \& Groeger, J. S. (2000). Transcutaneously tunnelled central venous lines in cancer patients: an analysis of device-related morbidity factors based on prospective data collection. Ann Surg Oncol, 7, 441-449.

Sheretz, R. J., Carruth, W. A., Marosok, R. D., Espeland, M. A., Johnson, R. A., \& Solomon, D. D. (1995). Contribution of vascular catheter material to the pathogenesis of infection: The enhanced risk of silicone in vivo. Journal of Biomedical Materials Research, 29, 635-645.

Sheth, N. K., Franson, T. R., Rose, H. D., Buckmire, F. L., Cooper, J. A., \& Sohnle, P. G. (1983). Colonisation of bacteria on polyvinyl chloride and teflon intravascular catheters in hospitalised patients. Journal of Clinical Microbiology, 18(5), 1061-1063.

Smith, J. R., Friedell, M. L., Cheatham, M. L., Martin, S. P., Cohen, M., \& Horowitz, J. (1998). Peripherally inserted central catheters revisited. American Journal of Surgery, 176(2), 208-211. 
Snelling, R., Jones, G., Figueredo, A., \& Major, P. (2001). Central venous catheters for infusion therapy in gastrointestinal cancer: a comparative study of tunnelled centrally placed catheters and peripherally inserted central catheters. Journal of Intravenous Nursing, 24(1), 38-47.

Stephens, L. C., Haire, W. D., \& Kotulak, G. D. (1995). Are clinical signs accurate indicators of the cause of central venous catheter occlusion? Journal of Parenteral and Enteral Nutrition, 19(1), 75-79.

Tebbs, S. E., Sawyer, A., \& Elliot, T. S. (1994). Influence of surface morphology on in vitro bacterial adherence to central venous catheters. Br J Anaesth, 72, 587-591.

Thompson, I., Melia, K., \& Boyd, K. (1996). Nursing Ethics. Edinburgh: Churchill Livingstone.

Tighe, M. J., Kite, P., Fawley, W. N., Thomas, D., \& McMahon, M. J. (1996). An endoluminal brush to detect the infected central venous catheter in situ: a pilot study. British Medical Journal, 313(7071), 1528-1529.

Todd, J. (1998). Peripherally inserted central catheters. Professional Nurse, 13(5), 297-302.

Todd, J., \& Hammond, P. (2004). Choice and use of peripherally inserted central catheters by nurses. Professional Nurse, 19(9), 493-497.

Tomford, J. W., Hershey, C. O., McLaren, C. E., Porter, D. K., \& Cohen, D. I. (1984). Intravenous therapy team and peripheral venous catheter-associated complications. A prospective controlled study. Arch Intern Med, 144(6), 1191-1194.

Vandenbroucke, J. P. (2002). The history of confounding. Social and Preventative Medicine (International Journal of Public Health), 47, 216-224.

Veenstra, D. L., Saint, S., Saha, S., Lumley, T., \& Sullivan, S. D. (1999). Efficacy of antiseptic-impregnated central venous catheters in preventing catheter-related bloodstream infection: A meta-analysis. JAMA, 281(3), 261-267.

Von Elm, E., Altman, D. G., Egger, M., Pocock, S. J., Gotzsche, P. C., \& Vandenbroucke, J. P. (2007). The strengthening the reporting of observational studies in epidemiology (STROBE) statement: guidelines for reporting observational studies. Bulletin of the World Health Organisation, 85(11), 867-872.

Walshe, L. J., Malak, S. F., Eagan, J., \& Sepkowitz, K. A. (2002). Complication rates among cancer patients with peripherally inserted central catheters. Journal of Clinical Oncology, 20(15), 3276-3281.

Wise, M., Richardson, D., \& Lum, P. (2001). Catheter tip position - a sign of things to come! J Vasc Access Devices, 6, 18-27.

Worth, L. J., Slavin, M. A., Brown, G. V., \& Black, J. (2007). Catheter-related bloodstream infections in haematology. Time for standardized surveillance? Cancer, 109(7), 1215-1226.

Yap, Y.-S., Karapetis, C., Lerose, S., Iyer, S., \& Koczwara, B. (2006). Reducing the risk of peripherally inserted central catheter line complications in the oncology setting. European Journal of Cancer Care, 15(4), 342-349.

Yebenes, J. C., Martinez, R., Serra-Prat, M., Sauca, G., Capdevilla, J., Balanzo, X. et al. (2003). Resistance to the migration of micro-organisms of a needle-free disinfectable connector. Journal of International Infection Control, 31(8), 462-464.

Yebenes, J. C., Vidaur, L. Serra-Prat, M., Sirvent, J., Battle, J. Matje, M., Bonet, A., \& Palomar, M. (2004). Prevention of catheter-related bloodstream infection 
in critically ill patients using disinfectable, needle-free connector: A randomised controlled trial. Journal of International Infection Control, 32(5), 291-295.

Yeung, C., May, J., \& Hughes, R. Infection rate for single-lumen vs. triple-lumen subclavian catheters. Infection Control and Hospital Epidemiology, 149, 1139-1143. 
Appendices 
Appendix 1 - Upper-South Regional Ethics Committee Approval

Health
and
Disability
Ethics
Committees

Upper South A Regional Ethics Committee

Ministry of Health

$4^{\text {th }}$ Floor, 250 Oxiord Terrace

PO Box 3877

Christchurch

Fione (03) 3723037

Fax (03) 3721015

6 February 2007

Ms Mary Fairhall

46 Kotare Street

Christchurch 8041

Dear Mary Fairhall,

Peripherally inserted central catheter (PICC)-related complications amongst

oncology patients. Retrospective review.

Investigator: Ms M Fairhall

Lacality: Christchurch Hospital

Ethics ref: URA/07/01/008

Thank you for the above application, which was considered by the Upper South A Regional Ethics Committee on 29 January 2007. Thank you also for attending the meeting, which was found to be most interesting and useful.

The above study is observational research, and has been given ethical approval by the Upper South A Ethics Committee.

\section{Accreditation}

The Committee involved in the approval of this study is accredited by the Health Research Council and is constituted and operates in accordance with the Operational Standard for Ethics Committees, April 2006.

\section{Final Report}

The study is approved. until 15 February 2007. A final report is required at the end of the study and a form to assist with this is available from the Administrator. If the study will not be completed as advised, please forward a progress report and an application for extension of ethical approval one month before the above date. Report forms are available from the administrator.

\section{Amendments}

It is also a condition of approval that the Committee is advised of any adverse events, if the study does not commence, or the study is altered in any way, including all documentation eg advertisements, letters to prospective participants.

Please quote the above ethics committee reference number in all correspondence.

It should be noted that Ethics Committee approval does not imply any resource commitment or administrative facilization by any healthcare provider within whose facility the research is to be carried out. Where applicable, authority for this must be obtained separately from the appropriate manager within the organisation. 
We wish you well with your study.

Yours sincerely

Alieke Diejrck

Upper South Â Ethics Commititee Âdministrator

Email: alieke_dierckx@moh.govt.nz 


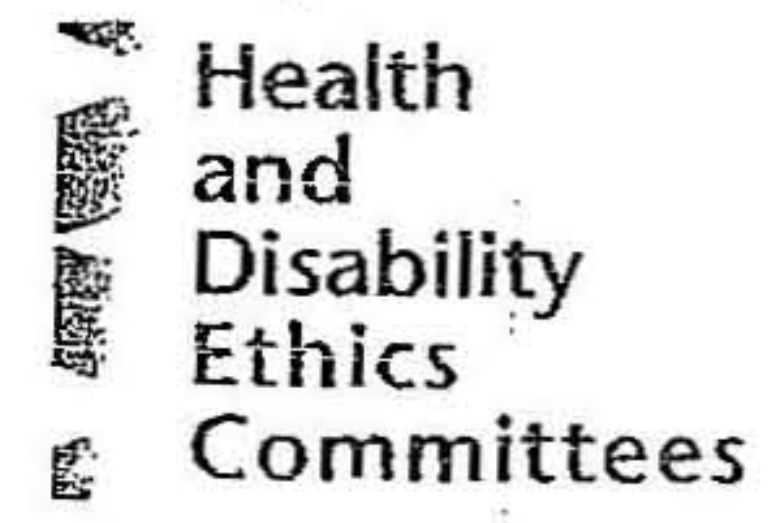

13 February 2007

Mis Mary Fairhall

46 Kotare Street

Christchurch 8041

Dear Mary Fairhall,

Peripheraliy inserted central catheter (PICC)-related complications amongst oncology patients. Retrospective review.

investigator: Ms M Fairhall

Localiťy: Christchurch Hospital

Ethics ref: URA $07 / 01 / 008$

Please accept my apologies for an error in my letter of 6 February 2007. The above study is approved until 15 February 2008.

Yours sincerely

Aliske Dierckx

Upper South A Ethics Committee Administrator
Upper South A Regional Ethics Committee

Ministry of Health $4^{\text {th }}$ Floor, 250 Oxford Terrace PO Box 3877

Christchurch

Phone (03) 3723037

Fax (03) 3721015 
29 January 2008

Ms Mary Fairhall

46 Kotare Street

Christchurch 8041

Dear Mary Fairhall,

Peripherally inserted central catheter (PICC)-related complications amongst oncology patients. Retrospective review:

investigator: Ms M Fairhall

Locality: Chrisicinurch Hospital

Ethics ref: URA/07/01/008

Request to include $13^{\text {th }}$ month's worth of data in analysis

Thank you for your letter of 15 January 2008 outlining the above request. This has been given approval by the Chairperson of the Upper South A Regional Ethics Committee under delegated authority.

Yours sincerely

Alieke Dierckx

Upper South A Ethics Committee Administrator 


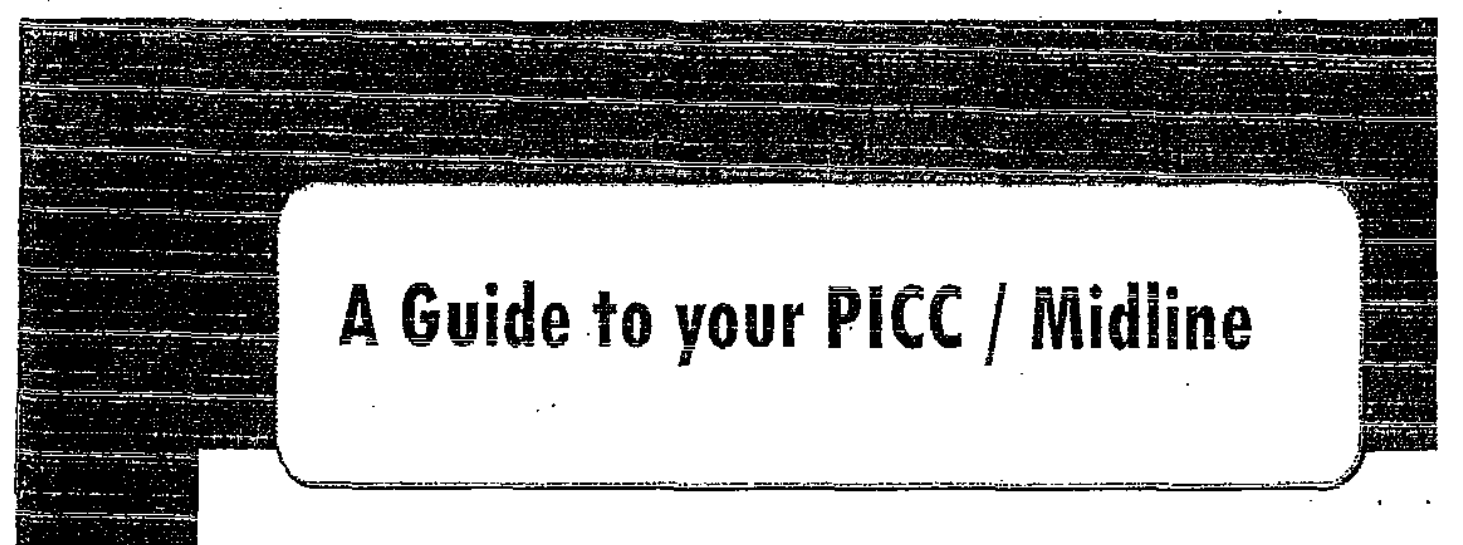

CLC2000 - Saline Lock

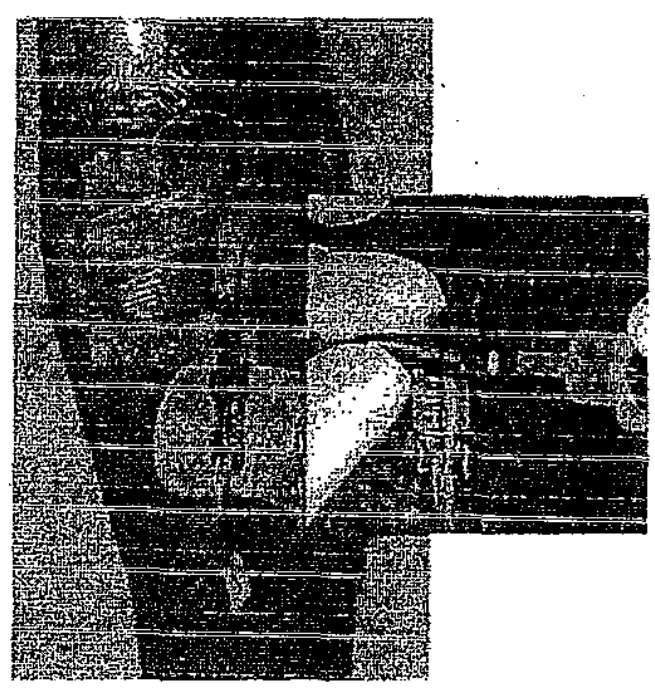

Canterbury

District Health Board

Te Poari Hauora ō Waitaha 


\section{Who to Contact}

You will be given the name of your nurse from the Service providing your care; should you require assistance.

\section{Nurse:}

$\square$ Nurse Maude Association: Phone: 3754200 (24 hours)

$\square$ Christchurch Hospital: Phone: 3640640

Ask for

$\square$ Other:

\section{Doctor / Department:}

The doctor responsible for your treatment is:

\section{Catheter Type and Details}

\begin{tabular}{|l|}
\hline Brand: \\
\hline Sizo: \\
\hline Flow rate \\
\hline Internal length: \\
\hline External iength: \\
\hline Insertion Date: \\
\hline
\end{tabular}




\section{A Guide to your PICC}

Supporting information for informed consent for health professionals and their patients.

\section{Introduction}

The Peripherally Inserted Central Catheter (PICC) or Midline Catheter is used for medium to long term drug therapy.

It is a very flexible tube (catheter) that is put into a vein in your arm. This catheter is threaded along the vein and rests in the major vessel, the superior vena cava, just outside the heart (PICC) or in a vein in the upper arm (Midline).

The Radiology Department nurse will place the catheter in your am.

The PICC/Midline catheter will allow medication of repeated doses of drug treatment without having multiple needles inserted into your veins. Blood samples can be taken from some PICC lines but not from Midlines.

This catheter can remain in your arm for a long period of time without requiring a change.

You will be able to continue with most daily activities. Some activities will be limited, such as repetitive movements of the arm with the PICC/Midline. Please discuss this with your nurse.

\section{Your catheter is a $\square$ PICC $\square$ MIDLINE}
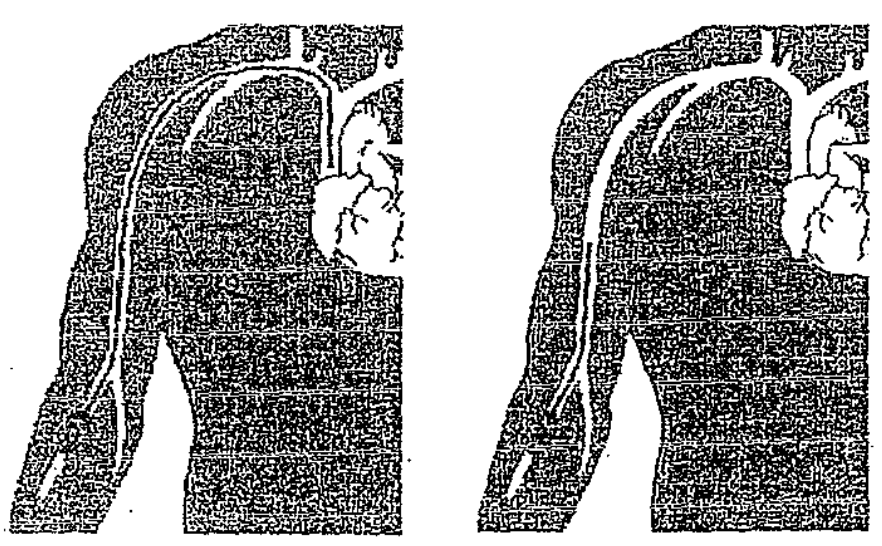


\section{The Parts of the Catheter}

1 The Catheter consists of a tube made of a material that allows for the cathieter to be left in place for a long time.

2 The Extension Set is the tubing attached to the external end of the catheter to increase the length to allow for ease of use. This usually stays on for the life of the catheter.

Attached to the Extension Set are two important parts:

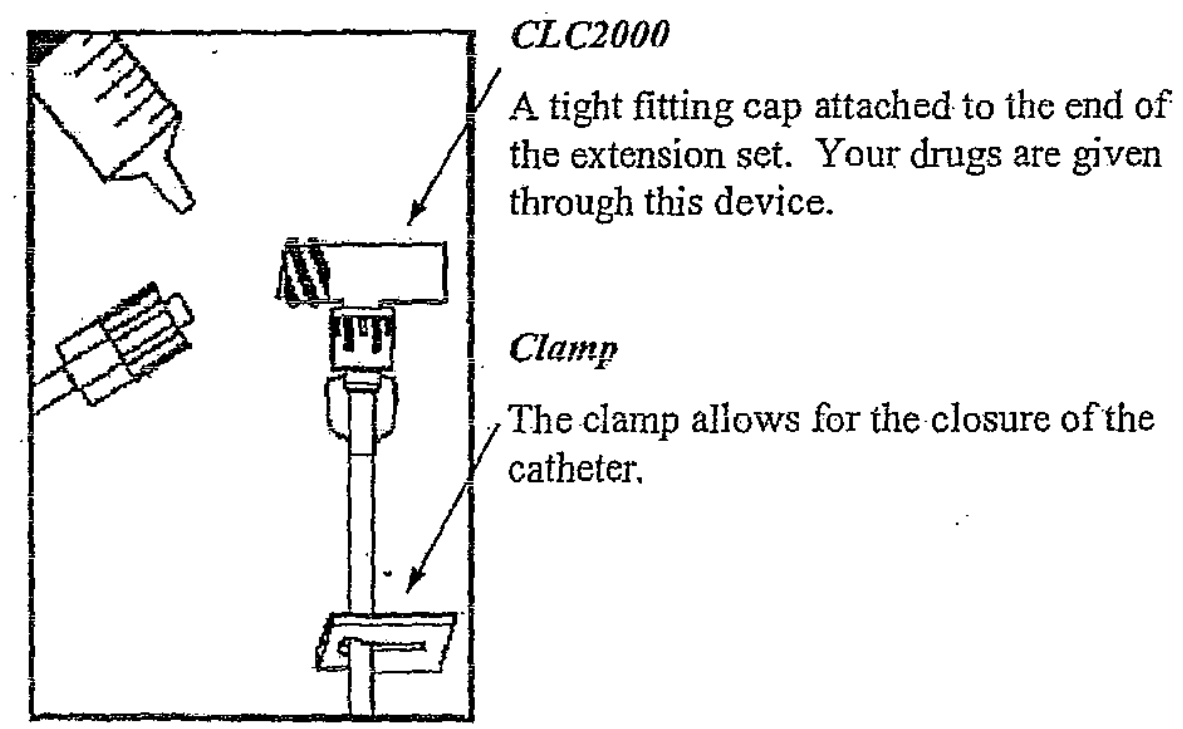

\section{Caring for your Catheter}

While in hospital your nurse will care for and help you learn about your catheter. Once you are discharged, your Outpatient/District Nurse will support you in the care of the catheter. 


\title{
Some important considerations to remember
}

\author{
Handwashing \\ Before receiving any care or treatment for your catheter, the nurse will \\ wash his/her hands. This is the most important method in preventing \\ infection.
}

\section{Ciean handling technique - aseptic}

The administration of your drugs is done by a clean (aseptic) method. This is to prevent contamination of the tube and is achieved by cleaning the CLC2000 with a sterile swab containing alcohol. The equipment used to give the drugs is sterile.

\section{Dressing}

The insertion site is covered with a dressing. The dressing will be changed weekly or sooner if it becomes loose, moist or falls off. Your nurse will redress the site.

The type of dressing materials used will allow for maximum protection of the catheter from infection. At all times the dressing must remain clean, dry and stick firmly to the skin.

Note: Do not get the dressing wet. You will be required to cover the dressing with a plastic bag or glad wrap and securely taped to prevent this happening when showering. Swimming is not permitted.

\section{Activities}

If your work or leisure pastimes involve a reasonable amount of activity, you will need to discuss this with your nurse. Activities that require strenuous arm use could cause sweiling of the arm with the catheter. This must be avoided.

\section{Keeping the catheter usable}

The catheter requires a continuous flow of medication into the catheter or regular placement of a Saline Lock to stop blockage when it is not in use. 


\section{When to seek help}

\section{Temperature}

You may need to take your temperature if you feel unwell or if directed by your nurse/doctor. If you develop a temperature above $38^{\circ} \mathrm{C}$ or develop chills or sweats.

Action: Contact your nurse or doctor.

\section{Adverse reaction}

When receiving drug therapy, a reaction may occur. This is very rare.

1) If you develop a rash, itchiness or swelling.

Action: See your doctor immediately.

2) Or suddenly become unwell, eg. develop a wheeze, short of breath, or experience chest pain.

Action: Ring for an ambulance.

\section{Dressing}

1) If the dressing is loose, moist or falls off.

Action: Cover the insertion site with sterile gauze and bandage. The dressing needs to be changed. Contact your nurse.

2) If you see any swelling, redness, blood, pus or other liquid under and around the dressing.

Action: Contact your nurse.

\section{Catheter blockage}

Resistance is felt when flushing the line.

Action: This is checked with gentle flushing. Do not use force. Contact your nurse.

\section{Câtheter or exíension set leakage}

Fluid is seen coming from the catheter or extension set.

Action: Check that the connections are right and clamp the catheter. Cover area of leakage with sterile gauze and tape or bandage. Contact your nurse. 


\section{Catheter - accidental remova!}

Action: Cover the insertion site with sterile gauze and bandage. You may need to press over the site to stop bleeding. Contact your nurse.

\section{Catheter - breakage}

The catheter is split or cut. Bleeding may occur or air may enter the catheter.

Action: Kink or clamp above the break to stop bleeding. You may need to hold the catheter to stop movement in or out of your arm.

Contact your nurse or ambulance immediately. If air has entered the catheter, lie flat and turn onto your left side; remembering to kink or clamp the catheter.

\section{Discharge with PICC Catheter}

Once you go home you will be assisted in the care of your catheter by one of the following methods:

\section{- District Nursing Service}

A nurse will visit you in your own home daily to change your infusion pump.

\section{- Caregiver/self administration}

Once you have undergone the training provided by the hospital and/or District Nurse, you or your caregiver can change the infusion pump each day. Your District Nurse will provide support to you. 


\section{Education for Self Aưministration}

Continue with the next section if you going onto the self-administration teaching package.

At the completion of training, you and your caregiver will be able to administer a Saline pulsatile flush.

You will be required to successfully complete administration of your medications. This is to be done 3 times under the supervision of your IV certified nurse.

Training may be commenced at any time when you are ready for learning. This can be commenced prior to discharge and the District Nurse will continue to support you with your education.

Your nurse will sign below as you complete each level of training and document achievement of education in your hospital or District Nursing notes.

Procedure observed $x 3$

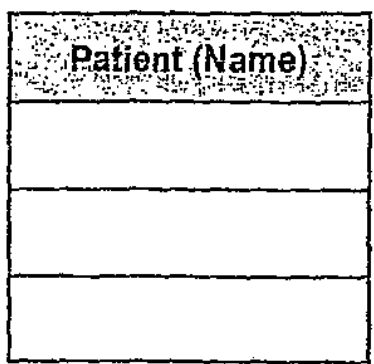

\section{CLC2000 Saline Lock}

To keep your catheter ready for use, a CLC2000 Saline flush is required once daily. The drug is called Sodium Chloride Injection BP $0.9 \%$ (Normal Saline). The catheter is flushed with $10 \mathrm{mls}$ of Normal Saline.

Your drug chart will have the prescription for the Saline flush.

The pulsatile Saline flush creates turbulence within the catheter to help keep the catheter clear of any particles.

Procedure completed $\times 3$

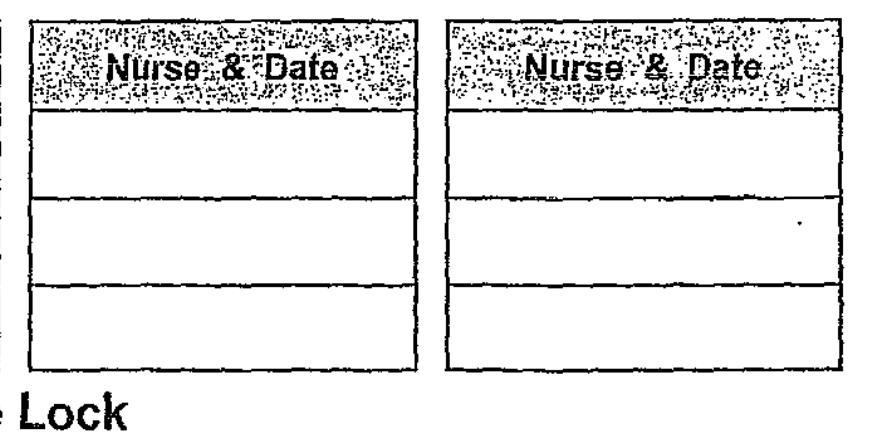

. 
Remember the aim of having this catheter is to keep it usable for future intravenous drug treatment.

\section{Set up}

Equipment

$\Rightarrow$ Drug treatment sheet

$\Rightarrow \quad$ Polyamp, normal saline $10 \mathrm{ml}$

Remember

$\Rightarrow$ Syringe $10 \mathrm{ml}$

$\Rightarrow \quad$ Swab containing alcohol

$\Rightarrow$ Biohazard bag

\section{Handwashing Technique}

Handwashing is essential prior to the giving of your drugs. This is the most effective means of preventing infection.

1 Remove all jewellery from arms and hands.

2 Wet your hands and forearms with warm running water.

3 Apply liquid soap and wash for 2 minutes. Pay attention to palms, between fingers, thumb and the backs of hands.

4 Rinse hands thoreughly with warm water. Ensure soap is completely removed.

5 If possible, turn taps off using clean paper towel.

6 First dry hands, keeping them upright, using clean hand towel and then dry your forearms.

\section{Preparation}

\section{Saline Flush}

1 Wash hands.

2 Twist top off the plastic ampoule.

3. Insert the syringe into the opening of the ampoule (polyamp).

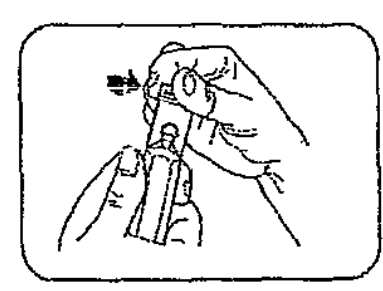


4 Withdraw fluid from polyamp into the syringe.

5 Expel any air remaining in the syringe. Check that there are no large air bubbles.

Your flush is ready.

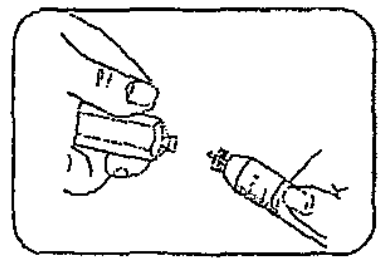

\section{Ádministration}

\section{Saline Flush}

1 Clean CLC2000 with alcohol swab. Allow to dry for 30 seconds.

2 Insert syringe containing normal saline into the CLC2000.

3 Saline flush. Firmly depress the plunger on the syringe using a quick push, pause, push, pause technique.

Stop if there is any inflammation, swelling, pain or you feel resistance.

4 Remove the syringe and discard into biohazard bag.

Saline flush prior is now completed.

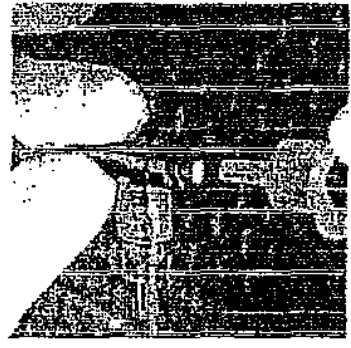

DO NOT CLAMP catheter or extension set while disconnecting syringe from CLC2000 as it will interrupt the positive displacement.

The CLC2000 and dressing are changed weekly by your nurse.

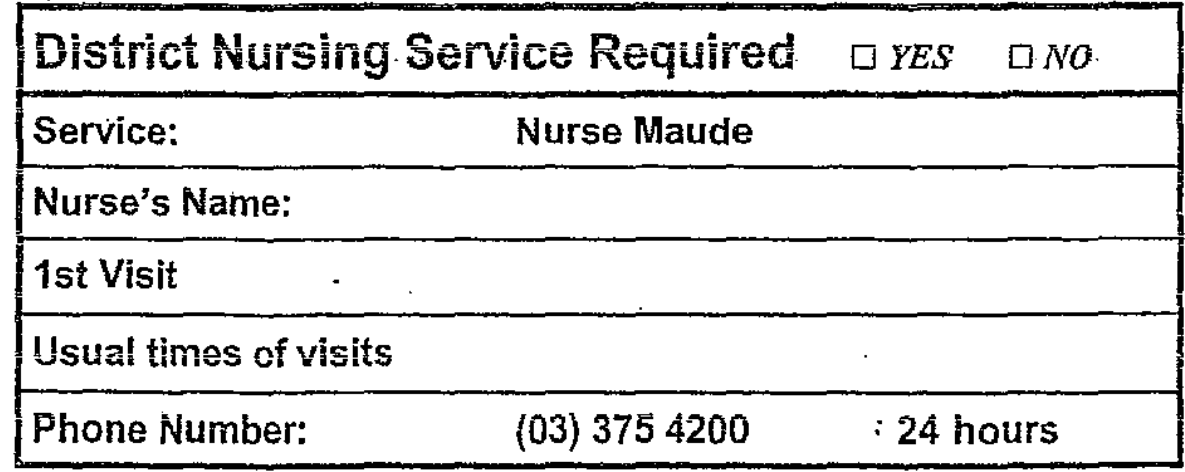




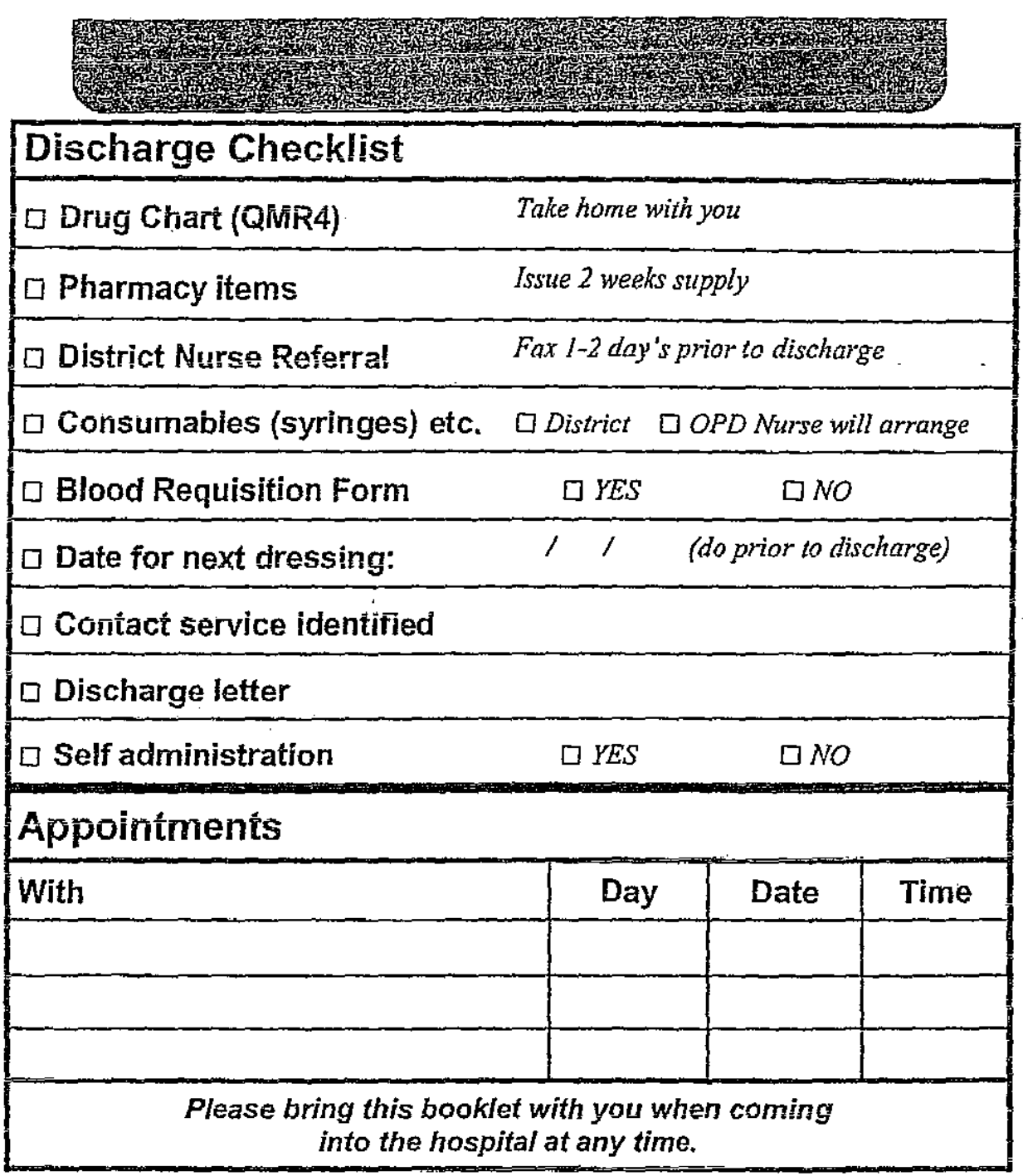

Issued By: Intravenous Antibiotic Service, Christchurch Hospital Date of Issue 2: 3 August 2004

Authorised By: Clinical Director, Infectious Diseases

g:lcontdocs 3rdlchchhosplivas IPICC MidilinelSaline Lock 403.pub Ref: 0403

\section{Canterbury}

\section{District Health Board \\ Te Poari Hauora ō Waitaha}


PATIENT LABEL

Name:

Consultant:

Hospital Number:
DOB:

Ward:

Phone No:
Reep this information in the Patiens "s; A duplicate copy will be kept in Radio

Contact Person: Radiology Nurses - Ph: 81410 or 80770

After Hours: Radiology Nurse On Call (via Telephone Office) 1800-2200 Mon-Fri and 0900-2200 weakrent

NSERTION

Date: _. I I T I _ Time: Inserted By: (Radiology Depa

CATAETER TYPE

Midline . Double Gange Size:__ Fr_____ Overail Length:

PICC Single Catheter Trimed? No

Iniemal Length:

Catheter Hourly Flow Rate $\mathrm{mishr}$

Pamp mishr

*Extemal Length:

*External Measurement of the catheter should to checked ually.

MSERTON SITE

Arm: Left - Right

Below AC

Position X-Ray Verined: Yes N

Veit: Basilic/Brochingl Madimn

Cephalic

Ávive AC

Position of PICC Tip: SVC

REASON FOR INSERTTON (eg A/B's, TPN)

NSERTION DETATS

\section{AllimgGS}

\section{RECONMENDPD SASE POUIINT}

$S=N \mathrm{NaCl} 10 \mathrm{nn}$. Flush:

(1) Pre Medication to be given as Bolus or Infusion

$A=$ Administer Medication:

(2) As Charterl

$\mathrm{S}=$ PULSATIE NaCl Flush:

(3) 0.9s 10nl Pulsafile Finsh

$\mathrm{H}=$ Heparin Lock:

(4) PICC: Hep/SaIine 50iu/ $5 \mathrm{ml}=5 \mathrm{ml}$ (ff accessing 8 houly or more often) Hep/Saline 500iu $/ 5 \mathrm{ml}=1.3 \mathrm{ml}$ (ff accessing 12 hourlv or less)

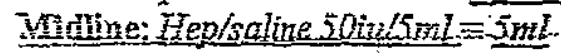

CATHETER TNORMATION (Keep dressing dry and occlusive at all times)

Dressing Change: Weekry or PRN if wetsoiled Type: TV3000, BroCLUSIYE ot Tegaderm

Luer Plug Change:72hrly and Dressing Change Method of securing: Steristrip Statlock-(Change: PRN.

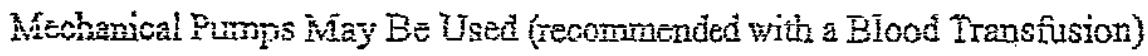

Biood Withdrawel: PICC-Yes Midine - No

NO BP CUFS OR TOURNIQUETS ON THE ARM WITH THE CATHETER 
Resource areas for CVAD expertise:

- PICC lines - Digital Subtraction Angiography (DSA) Radiology Departmer.

- Tunneiled central venous catheters - Haematology or Dialysis Services

- Implanted norts - Ward 25

- Short term CVC lines - General Surgery or ICU

- Paediatric insertions - Children's Haematology Oncology Clinic (CHOC)

- Dialysis Catheter - Dialysis Service

- Note: Some specialty areas use trained technicians to manage some CVADs, for example Dialysis Technologists.

\section{Non Tumelled percutaneous Central Venous Catheters (CVCs)}

Short iermi CVCs are percutaneous catheters placed by an intra-clavicular approach through the subclavian or jugular vein and secured by suturing. The catheter may remain in place for a few days to several weeks (refer to manufacture 's instinucions).

CVC's are inserted by experienced medical officers. This can be done at the bedside or in the ward treatment areas. Occasionally patients may be transferr to ICU for the procedure.

CVC's can be single, double, triple, or quadruple lumen. Inconpatible medications can be administered through different lumen at the same time. Note: CVCs positioned in the subclavian vein have a high risk of complications, eg. infection.

\section{Poripherally Inserted Central Catheters (PICC)}

The PICC is inserted into the cephalic, median or basilic vein (usially above th antecubital fossa) and advanced into the Superior Vena Cava. PICC's are inserted in Digital Subtraction Angiography (DSA) Radiology Department, by specialist mursing staff or by anaesthetists in Operating Theatre.

PICC lines may have a single or double lumen. They are increasingly being usi in acute care settings and for home IV therapy.

The PICC line has measured markings along the catheter. The external measurement of the catheter will be written in the clinical notes on insertion. This position should be checked daily.

PrCC lines can remain in place for to to a year (refer to manufacturer's instructions).

The Midline catheter is similar in anpearance to a PICC but the tip resides in th axillary region and does not advance into the Superior Vena Cava. A midine cannot be considered a central line, however its management is similar to PICC line management. 
Figure 2: Peripherally Inserted Central Catheter (PICC)

Tumielled CVADs

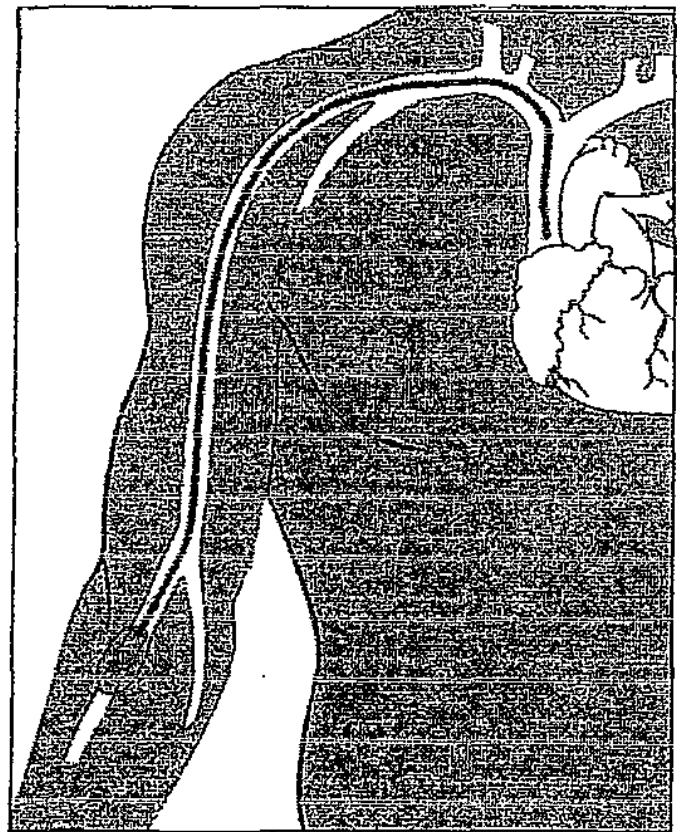

Tunnelled CVADs are introduced via the subclavian or jugular vein to a central venous position, then the catheter is tunnelled several centimetres under the skin and brought out through the skin to a suitable midchest exit site (anterior chest wall between sternum and nipple). Tunnelled catheters have a dacron cuff near the subcutaneous exit site of the catheter that anchors it in place and serves as a microbial barrier. Tunnelled catheters are inserted in DSA under local anaesthetic by radiologists and are intended for long term use (refer to manufacturer's instructions). Tunnelled CVADs include Hickmans and Tesio.

Figure 3: A Tumelled CVC

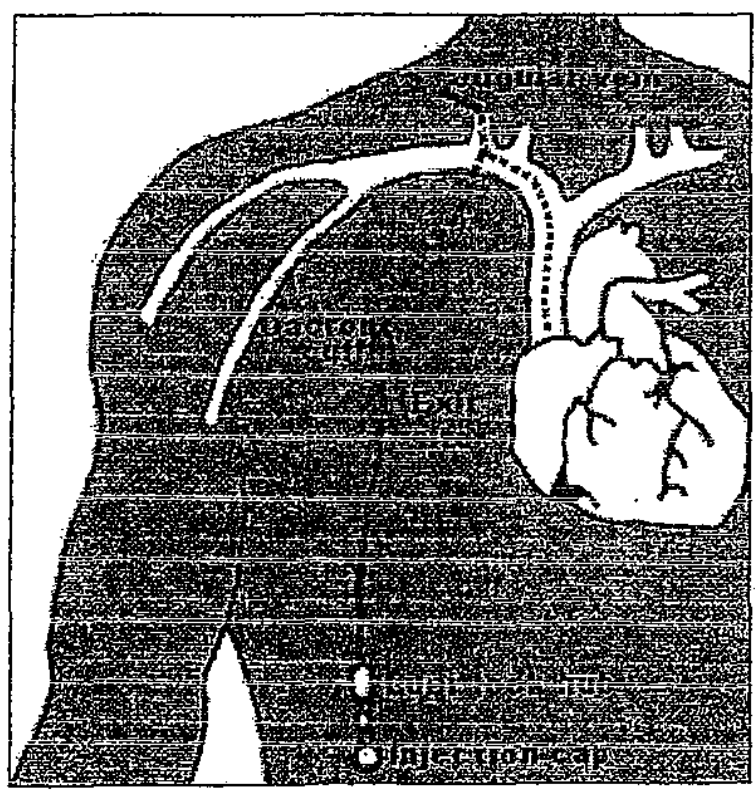

In Radiology, most tunnelled catheters are inserted via the Jugular, not the subclavian vein. Initially, tunnelled CVADs are sutured into place. Sutures are removed after $10-21$ days (refer local policy). 


\section{Implanted Ports}

Implanted ports are a completely closed system consisting of an implantable device with a drug reservoir, or port, with a self sealing system connected to an outlet catheter.

This device is surgically implanted under the skin. A subcutaneous pocket is created to hold the port. The port is usually placed under the pectoral muscles, or skin in the anterior chest wall below the clavicle. The catheter tip is then inserted into the desired central venous vessel, the port and catheter are then connected and the skin is closed. The self sealing septum can withstand up to 2000 nòn coring needle punctures (refer to manufacturer's instructions). They are used for patients requiring long-term intermittent therapy. A special angled non-coring needle is used to access the port reservoir. It is inserted through the skin into the port septum gently, until the base of the reservoir is sensed.

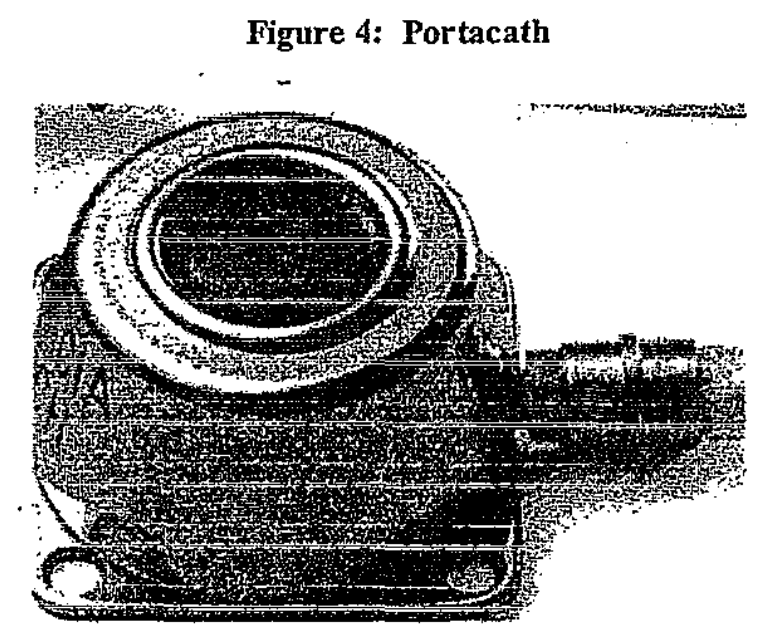

\section{Precautions with Central Venous Access Devices}

Position should be verified radiologically prior to accessing any newly inserted central line.

Avoid immersing any catheter in water. Dressings must be covered/sealed (ie. plastic bag or gladwrap) while patient showers/baths. 


\subsubsection{Glossary of Terms}

\section{Aseptic technique}

For the puipose of this document; aseptic technique refers to a procedural hand hygiene (2-3 minutes with antimicrobial soap), the use of sterile gloves, guards, instruments and equipment, and the maintenance of a non-touch technique throughout the entire procedure. An aseptic technique is required when breaking the line.

\section{'Breaking' the CVAD line}

Any instance where the catheter integrity is compromised, or the catheter lumen hub is open, is considered breaking the line and requires an aseptic technique, For example, administration set or needle free access device changes at the lumen hub, or accidental disconnection at the lummen hub.

\section{Centiral venows access device (CVAD)}

For the purpose of this document the term CVAD refers to:

- Non-Tunnelled central venous catheters (CVCs)

- Peripherally inserted central catheters (PICCs)

- Tunnelled central venous catheters (Hickman lines)

- Implanted poris

\section{Clean technique}

A clean technique refers to a social hand hygiene ( 15 seconds with antimicrobic soap) and the use of a non touch technique, in the instance where a CVAD is not being opened or broken and an aseptic technique is not required.

If there is any risk of blood or body fluid exposure non sterile gloves should be worn.

\section{Heparin lock}

The instillation of heparin into the CVAD lumen, using a positive pressure technique, whenever a CVAD is not in use continuously.

\section{Non-touch technique}

Technique whereby the key parts of the intravenous system or CVC are identified and not touched directly eg needles, syringe tips, line connections, exposed lumen or hub. 


\section{Positive pressure flush technique}

A method of clamping the CVAD as the last $0.2 \mathrm{~mL}$ of flush solution is being instilled, ie. maintaining a constant pressure on the syringe plunger whilst clamping. This will prevent reflux of blood into the tip of the device and help to prevent clotting and blockage.

\section{PlCC dressing pack}

This is a prepared pack, available from Supply Department, containing everything required for a CVAD dressing (except sterile gloves).

\section{Recommended cleaning solution \\ Chlorhexidine and Alcohol swab}

\section{Removal of heparin lock}

For the purpose of the document this refers to the removal and discard of the first 3-5mL blood from previously heplocked devices prior to flushing.

\section{Saline Paydatide Flush}

Using a 'push-pause-push' technique whilst flushing a CVAD with $0.9 \%$ sodium chloride; following administration of a medication, prior to connection of an administration set, following the withdrawal of a blood sannple and prior to heparin locking. This technique creates turbulence inside the catheter lumien aiding the removal of fibrin deposits and drug precipitation.

Figure 5: 'Turbulence' inside the Catheter limen

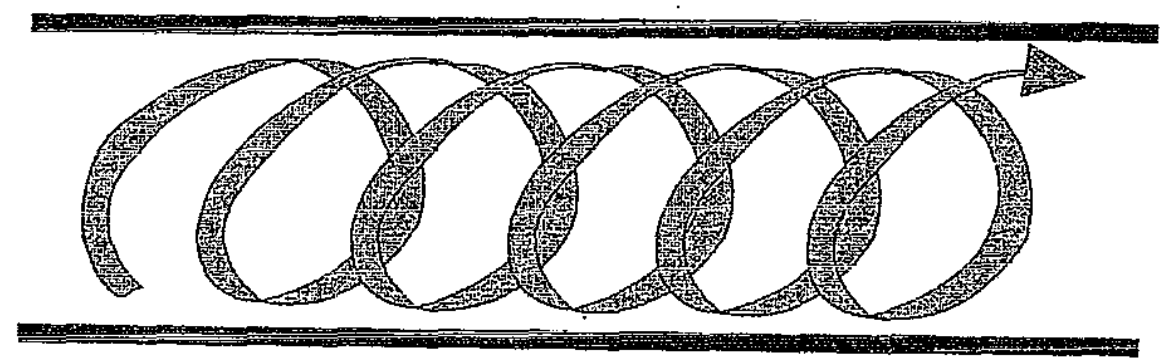

\section{Vaseactive}

A drug that is vasoactive constricts or dilates the veins. 


\subsubsection{CVAD Heplocking Procedure}

\section{Equipment}

$10 \mathrm{~mL}$ luer lock syringes as required

( $5 \mathrm{~mL}$ syringes may be used for heparin locking PICC lines)

Blunt plastic access cannulas

Heparin (as per heparin lock guidelines)

$10 \mathrm{~mL} 0.9 \%$ sodium chloride amponle

Chlorhexidine and alcohol swabs

Needle free access device (if required)

Sterile gloves and sterile gauze (if exposing catheter hub)

\begin{tabular}{|c|c|c|}
\hline 1 & $\begin{array}{l}\text { Ensure patient is informed and obtain } \\
\text { consent. }\end{array}$ & $\begin{array}{l}\text { To ensure the patient understands the } \\
\text { procedure. }\end{array}$ \\
\hline 2 & Social hand hygiene. & \\
\hline 3 & $\begin{array}{l}\text { Aseptically prepare the equipment and } \\
\text { assemble on clean dressing trolley or tray. } \\
\text { Procedural hand Hygiene. }\end{array}$ & \\
\hline 4 & $\begin{array}{l}\text { If using an existing needle free device, use } \\
\text { a clean technique and clean the needle free } \\
\text { access device with Chlorhexidine and } \\
\text { Alcohol swab and allow to evaporate. } \\
\text { or } \\
\text { If exposing the catheter hub, use an aseptic } \\
\text { technique, clean the catheter hub with } \\
\text { Chlorhexidine and Alcohol swab and allow } \\
\text { to dry. Place CVAD on sterile gauze. }\end{array}$ & Reduces the risk of introducing infection. \\
\hline 5 & $\begin{array}{l}\text { For heparin locked CVADs (excluding } \\
\text { preCs) withdraw previous heparin ( } 3-5 \mathrm{~mL}) \\
\text { and discard prior to flushing. If blood cannot } \\
\text { be withdrawn refer to medical team or } \\
\text { nursing staff from the clinical area } \\
\text { specialising in the particular catheter. }\end{array}$ & $\begin{array}{l}\text { Heparin should always be withơrawn and } \\
\text { discarded before attempting to flush line } \\
\text { as routine heparin administration has } \\
\text { been associated with bleeding disorders } \\
\text { and complications. } \\
\text { Or refer to local policy }\end{array}$ \\
\hline 6 & $\begin{array}{l}\text { Saline pulsatile flush. } \\
\text { Do not force if resistance is met. }\end{array}$ & $\begin{array}{l}\text { Creates turbulence inside the catheter } \\
\text { lumen aiding in the removal of fibrin } \\
\text { deposits and the prevention of drug } \\
\text { precipitation } \\
\text { Forcing may result in emboli or catheter } \\
\text { rupture. }\end{array}$ \\
\hline
\end{tabular}




\begin{tabular}{|c|c|c|}
\hline 7 & $\begin{array}{l}\text { Instil heparin solution using positive } \\
\text { pressure flush. }\end{array}$ & $\begin{array}{l}\text { To prevent backflow of blood into the } \\
\text { catheter. }\end{array}$ \\
\hline 8 & $\begin{array}{l}\text { Remove and dispose of equipment correctly. } \\
\text { Perform social hand hygiene. }\end{array}$ & \\
\hline 9 & $\begin{array}{l}\text { Record procedure and any variances in the } \\
\text { patient's clinical record. }\end{array}$ & \\
\hline
\end{tabular}




\subsubsection{Administration of Intermittent Intravenous Medication \\ Objective}

To safely and effectively administer medication through a CVAD.

\section{Personnel Authorised to Perform Procedure \\ IV Authorised staff who: \\ - Have demonstrated competency in dealing with CVAD's \\ - Have the approval of the CCN of the area \\ The correct sequence of administration for intermittent/bolus intravenous medication is: \\ S.A.S.H. (Saline, Administration, Saline, Heparin lock). \\ Equipment \\ Chlorhexidine and alcohol swabs \\ $10 \mathrm{~mL}$ luer lock syringes as required ( $5 \mathrm{~mL}$ syringes may be used to heparin lock PICC lines) \\ Sodium chloride $0.9 \%$ ampoules as required \\ Heparin (refer to Heparin Lock guidelines) \\ Blunt plastic cannula as required \\ Needle free access device (if required) \\ Sterile gloves and sterile gauze (if exposing catheter hub)}

\begin{tabular}{|c|c|c|}
\hline 1 & $\begin{array}{l}\text { Ensure patient is informed and obtain } \\
\text { consent. }\end{array}$ & $\begin{array}{l}\text { To ensure the patient understands the } \\
\text { procedure. }\end{array}$ \\
\hline 2 & Social hand hygiene. & \\
\hline 3 & $\begin{array}{l}\text { Aseptically prepare the equipment and } \\
\text { assemble on clean dressing trolley or tray. } \\
\text { Procedural hand hygiene. }\end{array}$ & \\
\hline \multirow[t]{2}{*}{4} & $\begin{array}{l}\text { If using an existing needle free device, use } \\
\text { a clean technique and clean the needle free } \\
\text { access device with Chlorhexidine and } \\
\text { Alcohol swab and allow to evaporate dry. } \\
\text { or }\end{array}$ & Reduces the risk of introducing infection. \\
\hline & $\begin{array}{l}\text { If exposing the catheter hub, use an aseptic } \\
\text { technique, clean the catheter hub with } \\
\text { Chlorhexidine and Alcohol swab and allow } \\
\text { to evaporate dry. Place CVAD on sterile } \\
\text { gauze. }\end{array}$ & 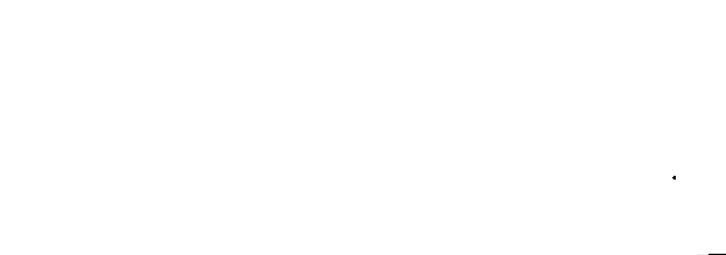 \\
\hline
\end{tabular}




\begin{tabular}{|c|c|c|}
\hline \multicolumn{2}{|c|}{ 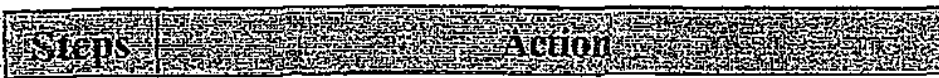 } & (4) \\
\hline 5 & $\begin{array}{l}\text { For heparin locked CVADs (excluding } \\
\text { PICCs), withdraw previous heparin }(3-5 \mathrm{~mL}) \\
\text { and discard prior to flushing. } \\
\text { If blood cannot be withdrawn refer to } \\
\text { medical team or nursing staff experienced } \\
\text { with the particular catheter. }\end{array}$ & $\begin{array}{l}\text { Heparin should always be withdrawn and } \\
\text { discarded before attempting to flush line } \\
\text { as routine heparin administration has } \\
\text { been associated with bleeding disorders } \\
\text { and complications. }\end{array}$ \\
\hline 6 & $\begin{array}{l}\text { Saline pulsatile flush. } \\
\text { Do not force if resistance is met. }\end{array}$ & $\begin{array}{l}\text { Creates turbulence inside the catheter } \\
\text { lumen aiding the removal of fibrin and } \\
\text { drug precipitation. } \\
\text { Forcing may result in emboli or catheter } \\
\text { rupture. }\end{array}$ \\
\hline 7 & Administer medication. & $\begin{array}{l}\text { Follow procedure and administration as } \\
\text { per Drug Treatment Chart and } \\
\text { manufacturer's instructions. }\end{array}$ \\
\hline 8 & Firm Saline pulsatile flush. & $\begin{array}{l}\text { Creates turbulence inside the catheter } \\
\text { lumen aiding the removal of fibrin and } \\
\text { drug precipitation. }\end{array}$ \\
\hline 9 & $\begin{array}{l}\text { Instil 프eparin solution using positive } \\
\text { pressure flush. }\end{array}$ & $\begin{array}{l}\text { To prevent backflow of blood into the } \\
\text { catheter }\end{array}$ \\
\hline 10 & $\begin{array}{l}\text { Remove and dispose of equipment correctly. } \\
\text { Perform hand hygiene. }\end{array}$ & \\
\hline 11 & $\begin{array}{l}\text { Record procedure and any variances in the } \\
\text { patient's clinical record. }\end{array}$ & \\
\hline
\end{tabular}




\subsubsection{Administration of Continuous Infusions}

\section{Objectives}

To safely and effectively administer an intravenous infusion via a CVAD.

\section{Personnel Authorised to Perform Procedure}

Registered Nurse/Anaesthetic Technicians with CDHB IV certification who:

- Have demonstrated competency in dealing with CVAD's

- Have the approval of the CCN/NM of the area

All patients receiving continuous intravenous fluids (including blood products) via a CVAD must have their infusion controlled by an infusion pump.

\section{Exceptions:}

Infusion pumps damage the fragile cell membrane of platelets, therefore it is recommended that platelets be gravity fed via an infusion set for blood products.

\section{Equipment}

Chlorhexidine and alcohol swab

$10 \mathrm{~mL}$ luer lock syringes as required

$10 \mathrm{~mL} 0.9 \%$ sodiun chloride ampoule

Blunt plastic cannula

Threaded lock cannula

IV Administration set

Infusion pump

Fluid/Drug for administration

\section{Connecting an Intravenous Infusion}

\begin{tabular}{|c|c|c|}
\hline 1 & $\begin{array}{l}\text { Ensure patient is informed and obtain } \\
\text { consent. }\end{array}$ & $\begin{array}{l}\text { To ensure the patient understands the } \\
\text { procedure. }\end{array}$ \\
\hline 2 & Procedural hand hygiene. & \\
\hline 3 & $\begin{array}{l}\text { Prime the IV administration set and the } \\
\text { threaded lock cannula. }\end{array}$ & \\
\hline 4 & $\begin{array}{l}\text { Clean the access port with Chlorhexidine } \\
\text { and alcohol swab. Allow to evaporate dry } \\
\text { for } 30 \text { seconds. }\end{array}$ & $\begin{array}{l}\text { Reduces the risk of introducing infection } \\
\text { and provides optimal asepsis. }\end{array}$ \\
\hline
\end{tabular}




\begin{tabular}{|c|c|c|}
\hline 5 & $\begin{array}{l}\text { For heparin locked CVADs (excluding } \\
\text { PICCs), withdraw previous heparin ( } 3-5 \mathrm{~mL} \text { ) } \\
\text { and discard prior to flushing. If blood } \\
\text { cannot be withdrawn refer to medical team } \\
\text { or nursing area specialising in the particular } \\
\text { catheter. } \quad:\end{array}$ & $\begin{array}{l}\text { Heparin should always be withdrawn and } \\
\text { discarded before attempting to flush line } \\
\text { as routine heparin administration has } \\
\text { been associated with bleeding disorders } \\
\text { and complications. } \\
\text { (or refer to local policy) }\end{array}$ \\
\hline 6 & Saline pulsatile flush. & \\
\hline 7 & Do not force if resistance is met. & $\begin{array}{l}\text { Forcing may result in emboli or catheter } \\
\text { rupture. }\end{array}$ \\
\hline 8 & $\begin{array}{l}\text { Attach the primed line by connecting the } \\
\text { threaded lock cannula to the needle free } \\
\text { access device. }\end{array}$ & $\begin{array}{l}\text { The threaded lock cannula is preferred to } \\
\text { the lever lock cannula for CVAD's as it is } \\
\text { more secure and comfortable for the } \\
\text { patient. }\end{array}$ \\
\hline 9 & $\begin{array}{l}\text { Programme infusion pump. Open clamps } \\
\text { and commence infusion at prescribed rate. }\end{array}$ & \\
\hline 10 & $\begin{array}{l}\text { Ensure that all tubing is labelled as per } \\
\text { hospital standards. }\end{array}$ & \\
\hline 11 & $\begin{array}{l}\text { Remove and dispose of waste equipment } \\
\text { correctly. Perform hand hygiene. }\end{array}$ & \\
\hline 12 & $\begin{array}{l}\text { Record procedure and any variance in the } \\
\text { patient's clinical record. }\end{array}$ & \\
\hline
\end{tabular}

\section{Disconnecting an Intravenous Infusion}

\begin{tabular}{|c|c|c|}
\hline 1 & $\begin{array}{l}\text { Ensure patient is informed and obtain } \\
\text { consent. }\end{array}$ & $\begin{array}{l}\text { To ensure the patient understands the } \\
\text { procedure. }\end{array}$ \\
\hline 2 & Use a clean technique throughout procedure. & \\
\hline .3 & $\begin{array}{l}\text { Clamp both the catheter and the infusion } \\
\text { line. }\end{array}$ & \\
\hline . 4 & Disconnect the infusion. & \\
\hline 5 & $\begin{array}{l}\text { Clean the access port with Chlorheridine } \\
\text { and alcohol swab. Allow to evaporate dry } \\
\text { for } 30 \text { seconds. }\end{array}$ & $\begin{array}{l}\text { Reduces the risk of introducing infection } \\
\text { and provides optimal asepsis. }\end{array}$ \\
\hline 6 & $\begin{array}{l}\text { If disconnecting vasoactive drug, } \\
\text { withdraw } 3 \mathrm{~mL} \text { of fluid from the catheter } \\
\text { prior to flushing. }\end{array}$ & $\begin{array}{l}\text { The dead space in the catheter contains } \\
\text { the remains of the infusion. If flushing } \\
\text { without withdrawing the patient can get a } \\
\text { bolus of medication. }\end{array}$ \\
\hline
\end{tabular}




\begin{tabular}{|c|c|c|}
\hline 7 & Firm Saline nulsatile flush & \\
\hline 8 & $\begin{array}{l}\text { Instil Heparin solution using positive } \\
\text { pressure flush. }\end{array}$ & $\begin{array}{l}\text { To prevent backflow of blood into the } \\
\text { catheter. }\end{array}$ \\
\hline 9 & $\begin{array}{l}\text { Remove and dispose of equipment correctly. } \\
\text { Perform hand hygiene. }\end{array}$ & \\
\hline 10 & $\begin{array}{l}\text { Record procedure and any variances in the } \\
\text { patient's clinical record. }\end{array}$ & \\
\hline \multicolumn{3}{|c|}{$\begin{array}{l}\text { If problems experienced with PICC line, contact DSA Nurse via Telephone Office (available } \\
\text { until } 2200 \text { hours). }\end{array}$} \\
\hline
\end{tabular}




\subsubsection{CVAD Dressing Changes}

\section{Objectives}

To ensure that CVAD exit site dressings are managed in a safe and effective manner.

\section{'Personnel Authorised to Perform Procedure}

IV Authorised Staff who:

- Have demonstrated competency in dealing with CVAD's

- Have the approval of the CCN/NM of the area

\section{Frequency}

Every seven days in the case where a transparent high moisture vapour permeable dressing is used or whenever integrity of the dressing is compromised, or blood is present at the exit site.

\section{PICC Lines}

- Sterile steri strips and stat lock should be used to secure the PICC in addition to the transparent, high moisture vapour permeable dressing.

- Replace stat lock pad as required.

- Measure catheter length to check for migration and document. Tliis measurement should be to the marked catheter length of the PICC, not to the catheter hub.

- Mechanical phlebitis is more likely to occur within the first 7 days following insertion of the PICC. It can usually be resolved within 48 hours by the application of heat to the upper arm for 20 minutes, 3 times a day; rest and elevate the arm.

\section{unuelled CVAD}

- Application of a biopatch around the catheter exit site is recommended until the skin tunnel has healed (normally 4 weeks).

- Removal of sutures:

- Entry site sutures after 10 days.

- Exit site sutures should be removed at 21 days by which time the skin tunnel should have granulated around the dacton cuff.

- Additional anchoring with an appropriate device may be indicated for some patients (refer local policy). 


\section{CVCs}

- Use two transparent bioocclusive dressings "sandwiched" together to maintain integrity of dressing and allow neck movement.

\section{Implanted Ports}

Once the wound overlying the skin incision has healed, there is no need for further dressings, unless the patient requires a continuous infusion. In this case a dressing will be required to stabilise the infusion device whilst it remains insitu.

\section{CVAD Dressing Procedure}

\section{Equipment}

PICC dressing pack or equivalent

Sterile gloves

New stat lock pad (if required)

\section{Prior to Procedure}

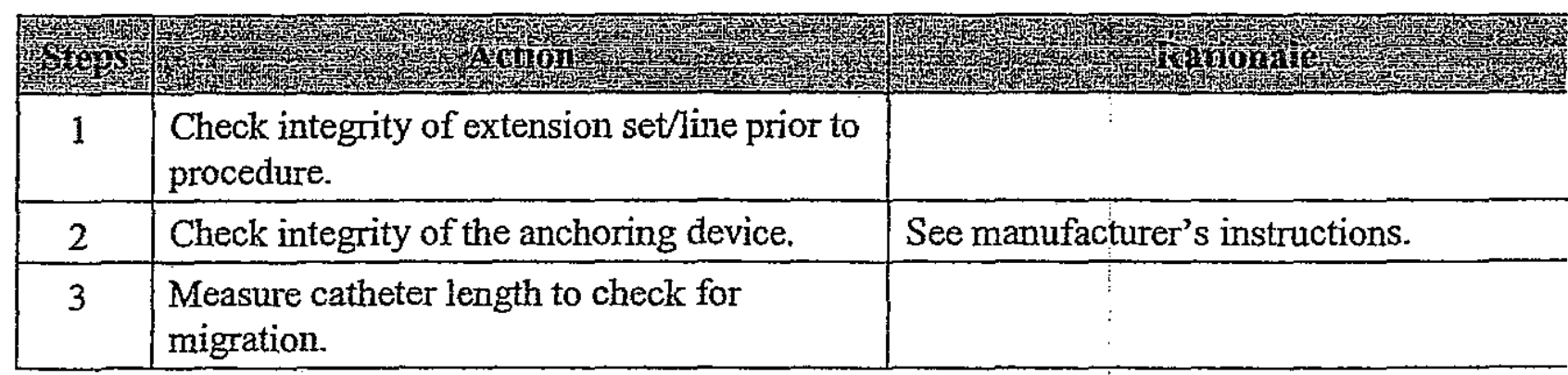

\section{Procedure}

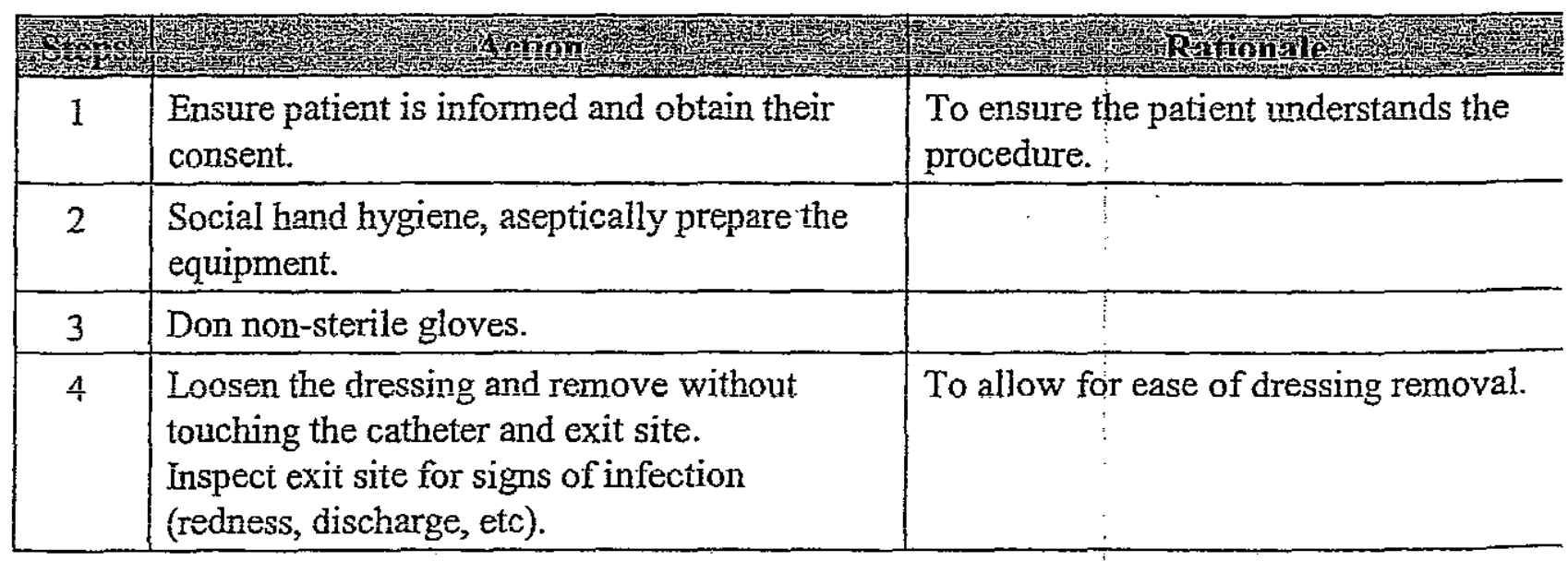




\begin{tabular}{|c|c|c|}
\hline 5 & Procedural hand hygiene. & \\
\hline 6 & $\begin{array}{l}\text { Don sterile gloves. Remove loosened dressing } \\
\text { with forceps, and discard. }\end{array}$ & \\
\hline 7 & $\begin{array}{l}\text { Clean catheter site with chlorhexidine and } \\
\text { alcohol swab, swab in a circular motion. Start } \\
\text { from the exit site, extending out to the area } \\
\text { that will be covered by the dressing. Do not } \\
\text { return to the catheter exit site with the same } \\
\text { swab. }\end{array}$ & $\begin{array}{l}\text { To reduce the risk of introducing } \\
\text { infection and provide optimal asepsis. }\end{array}$ \\
\hline 8 & $\begin{array}{l}\text { Allow to evaporate dry completely, then } \\
\text { repeat. } \\
\text { - Secure PICC line with steristrips } \\
\text { - Apply biopatch if indicated. }\end{array}$ & $\begin{array}{l}\text { To additionally anchor line. } \\
\text { Reduces risk of catheter colonisation } \\
\text { and catheter related blood stream } \\
\text { infections. }\end{array}$ \\
\hline 9 & $\begin{array}{l}\text { Apply transparent high moisture vapour } \\
\text { permeable rate dressing. } \\
\text { Lines can be looped under the dressing for } \\
\text { extra security. Leave extension set / } \\
\text { injection port exposed. }\end{array}$ & $\begin{array}{l}\text { To reduce the risk of introducing } \\
\text { infection and provide optimal asepsis. } \\
\vdots\end{array}$ \\
\hline 10 & $\begin{array}{l}\text { Dispose of all waste as per policy and perform } \\
\text { hand hygiene. }\end{array}$ & \\
\hline 11 & $\begin{array}{l}\text { Document: } \\
\text { - Date of dressing change. } \\
\text { - Physical condition of the site. } \\
\text { - Any nursing intervention. } \\
\text { - Catheter measurements. }\end{array}$ & 、 \\
\hline 12 & $\begin{array}{l}\text { Report to medical staff signs of infection or } \\
\text { changes in skin integrity. }\end{array}$ & . \\
\hline
\end{tabular}




\subsubsection{Extension Set Change}

\section{Objective}

To ensure the safe and effective change of a CVC extension set

\section{Personnel Authorised to Perform Procedure}

IV Authorised staff who:

- Have demonstrated competency in dealing with portacaths

- Have the approval of the CCN/NM of the area

\section{Frequency}

All lines require an extension set with a clamp device if the line itself does not have a clamp. The extension set is connected under strict asepsis at the time of line insertion and is therefore considered as part of the catheter, remaining insitu for the life of the catheter.

Replacement of the extension set is only required if its integrity is compromised ie. leakage, overclamping, contamination, use of blood products or Parental Nutrition, etc.

Once the extension set has been replaced it can no longer be considered part of the catheter and it then becomes necessary to rontinely replace the extension set weekly.

\section{Tquipment}

Dressing pack

Chlorhexidine and alcohol swab

Extension set.

Sodium chloride $0.9 \%$ ampoules.

$10 \mathrm{~mL}$ Syringe luer lock as required.

Needle free Access Port

Blunt plastic cannula as required.

Heparin (refer to page Heparin lock guidelines)

Sterile gloves 


\begin{tabular}{|c|c|c|}
\hline (1) & 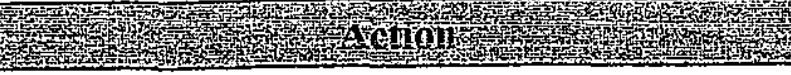 & 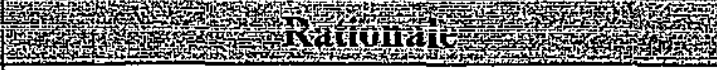 \\
\hline 1 & $\begin{array}{l}\text { Ensure patient is informed and obtain their } \\
\text { consent. }\end{array}$ & $\begin{array}{l}\text { To ensure the patient understands the } \\
\text { procedure. }\end{array}$ \\
\hline 2 & $\begin{array}{l}\text { Social hand hygiene. } \\
\text { Aseptically prepare the equipment. } \\
\text { Draw up the Sodium Chloride ampoule into } \\
\text { 10mL syringe. Place syringe in an accessible, } \\
\text { clean area. }\end{array}$ & \\
\hline 3 & $\begin{array}{l}\text { Procedural hand hygiene. } \\
\text { Don sterile gloves. }\end{array}$ & $\begin{array}{l}\text { An aseptic technique is required when } \\
\text { breaking the line. }\end{array}$ \\
\hline 4 & $\begin{array}{l}\text { Screw the sterile access port onto the new } \\
\text { extension set. }\end{array}$ & \\
\hline 5 & $\begin{array}{l}\text { Prime the set by injecting sodium chloride } \\
\text { into the set and clamp. }\end{array}$ & $\begin{array}{l}\text { Priming the set eliminates all air from the } \\
\text { set. }\end{array}$ \\
\hline 6 & $\begin{array}{l}\text { Check that the extension set attached to the } \\
\text { needle is clamped. }\end{array}$ & Reduces the risk of air embolism. \\
\hline 7 & $\begin{array}{l}\text { Remove existing extension set using sterile } \\
\text { gauze. } \\
\text { (Keep the extension set below the level of } \\
\text { the heart.) }\end{array}$ & Reduces the risk of air embolism. \\
\hline 8 & Attach new extension set. & \\
\hline 9 & Saline pulsatile flush. & \\
\hline 10 & $\begin{array}{l}\text { Instil Heparin lock using a positive pressure } \\
\text { flush. }\end{array}$ & \\
\hline 11 & Remove gloves and perform hand hygiene. & \\
\hline 12 & Remove and dispose of equipment correctly. & \\
\hline 13 & $\begin{array}{l}\text { Record procedure and any variances in the } \\
\text { patient's clinical record. }\end{array}$ & \\
\hline
\end{tabular}




\subsubsection{Blood Sampling from a CVAD}

Objective

To safely obtain blood samples from a CVAD.

ursonnel Authorised to Perform Procedure

IV A.uthorised staff who:

- have demonstrated competence in dealing with CVAD's.

- have the approval of the CCN/NM of the area.

\section{iding Principles}

- Use of any catheter for blood sampling increases the risk of sepsis and of occluding the inner lumen.

- When withdrawing blood from a PICC line do not use vacutainers or large syringes (above $5 \mathrm{~mL}$ ) as they yield high negative pressure causing potential catheter collapse.

- Coagulation testing and aminoglycoside levels are not reliable when collected through a central catheter. To promote greater accuracy a 2-10 $\mathrm{mL}$ discard is required prior to obtaining samples. (Depending on CVAD)

- The lumen of the CVAD must be greater than 22G. Withdrawing blood through a lumen smaller than this may damage the platelets and result in altered laboratory results.

- A CVAD inserted for Dialysis should be managed by renal speciality staff. (CVADs for Dialysis should not be used for blood sampling or administration of $I V$ fluids or medications.)

\section{Xquipment}

Non sterile gloves

Chlorhexidine and alcohol swab

$5 \mathrm{~mL}$ syringes as required (number depends on how much blood needed)

Blood tubes

Heparin lock syringes and flush solutions as required (refer to heparin lock guidelines)

Biunt plastic access carnulas

New needle free access port (if required)

Sterile gloves and dressing towel (if exposing the catheter hub) 


\begin{tabular}{|c|c|c|}
\hline 1 & $\begin{array}{l}\text { Ensure patient is infonner and obtain their } \\
\text { consmint. }\end{array}$ & $\begin{array}{l}\text { To ensure the patient understands the } \\
\text { procedure. }\end{array}$ \\
\hline 2 & $\begin{array}{l}\text { Social hand hygiene, aseptically prepate the } \\
\text { equipment and assemble on clean dressing } \\
\text { trolley or tray. }\end{array}$ & \\
\hline 3 & $\begin{array}{l}\text { Clamp any infusions in progress via all } \\
\text { lumens. Give saline flush, wait one minute. }\end{array}$ & \\
\hline 4 & $\begin{array}{l}\text { If using an existing needle free device, use } \\
\text { a clean techrique and clean he needle fres } \\
\text { access device with chlorhexidine and } \\
\text { alcohol swatb and allow to evaporate dry. } \\
\text { Or } \\
\text { If exposing the catheter hub (preferable for } \\
\text { PICCs) use an aseptic technique to remove } \\
\text { luer, clean the catheter hub with } \\
\text { Chlorinexidine and Alcohol swab and allow } \\
\text { to evaporate dry. Place CVAD on sterile } \\
\text { gauze. } \\
\text { Unclamp selected lumen and aspirate 5- } \\
\text { lomL of blood and reclamp lumen. } \\
\text { Discard blood unless blood cultures are } \\
\text { to be taken. This initial blood draw } \\
\text { should be used as the specimen for the } \\
\text { blood cultures. } \\
\text { Obtain blood specinens as requested, by } \\
\text { slowly withdrawing blood. Clamp } \\
\text { lumen when changing syringes. }\end{array}$ & To ensure laboratory results are accurate. \\
\hline 5 & $\begin{array}{l}\text { Saline pulsatile flush }(\times 2) \text {. Ensure no blood } \\
\text { remains in the needle free access device. } \\
\text { (or change for PlCCs) }\end{array}$ & $\begin{array}{l}\text { Creates turbulence inside the catheter } \\
\text { hmen aiding the removal of fibrin and } \\
\text { drug precipitaiton. }\end{array}$ \\
\hline 6 & $\begin{array}{l}\text { Recommence infusion or if line not in use } \\
\text { instil heparin solution using a positive } \\
\text { pressure fush technique. }\end{array}$ & $\begin{array}{l}\text { To prevent backflow of blood into the } \\
\text { catheter. }\end{array}$ \\
\hline 7 & $\begin{array}{l}\text { Remove and dispose of equipment correctly. } \\
\text { Peifom hand higivise }\end{array}$ & : \\
\hline 8 & $\begin{array}{l}\text { Record procedure and any variances in the } \\
\text { patient's clinical record. }\end{array}$ & \\
\hline
\end{tabular}




\subsubsection{Removal of Non-Cuffed CVC \& PICC in the Ward}

\section{Objective}

To safely remove a uon cuffed CVC in the ward setting.

Note:

- Tunnelled cuffed catheters are removed by Medical Officers/Anaesthetist/ Haematology/Oncology Registrar.

- Patient is usually sedated prior to procedure.

- Dialysis catheters are to be removed by renal specialty staff, Medical Officers/Anaesthetist.

\section{Personnel Authorised to Perform Procedure}

IV Authorised staff who:

- have demonstrated competence in dealing with CVC's and PICC's.

- have the approval of the CCN of the area.

\section{Fquipment}

Dressing pack.

Sterile gloves

Chlorhexidine and alcohol swab

Suture removal scissors/blade (if required)

IV pressure dressing.

\begin{tabular}{|c|l|l|l|}
\hline 1 & $\begin{array}{l}\text { Ensure patient is informed and obtain their } \\
\text { consent. }\end{array}$ & $\begin{array}{l}\text { To ensure the patient understands the } \\
\text { procedure. }\end{array}$ \\
\hline 2 & Cease any infusions. & \\
\hline 3 & $\begin{array}{l}\text { Position patient in the supine position, with } \\
\text { head down if this can be tolerated. }\end{array}$ & $\begin{array}{l}\text { Increases CVP pressure preventing air } \\
\text { being aspirated into the venous system. }\end{array}$ \\
\hline 4 & $\begin{array}{l}\text { Social hand hygiene. } \\
\text { Aseptically prepare equipment. } \\
\text { Remove the CVAD dressing without } \\
\text { touching catheter entry site and discard. }\end{array}$ & \\
\hline 5 & \begin{tabular}{l} 
Procedural hand hygiene. \\
\hline 6
\end{tabular} & $\begin{array}{l}\text { Clean catheter site with Chlorhexidine and } \\
\text { alcohol swab in a circular motion. Start } \\
\text { from the exit site, extending out. Allow to } \\
\text { evaporate dry for } 30 \text { seconds. }\end{array}$ \\
\hline
\end{tabular}




\begin{tabular}{|c|c|c|}
\hline 7 & $\begin{array}{l}\text { Cut suture if present. } \\
\text { Note: During removal of CVAD instruct } \\
\text { patient to perform the valsalva movement } \\
\text { (trying to breathe out with the glottis } \\
\text { closed). If this is not possible, patient is } \\
\text { asked to hold their breath. Removal } \\
\text { performed on expiration. Keep the catheter } \\
\text { lextension set below the level of the heart. }\end{array}$ & $\begin{array}{l}\text { To maintain positive pressure and lessen } \\
\text { the risk of drawing air into the vein. }\end{array}$ \\
\hline 8 & $\begin{array}{l}\text { Remove the catheter by placing sterile gauze } \\
\text { over the catheter site and withdrawing } \\
\text { catheter in a slow constant motion (no } \\
\text { resistance should be felt). }\end{array}$ & $\begin{array}{l}\text { Catheter fracture and embolisation can } \\
\text { occur if the CVAD is removed against } \\
\text { resistance }\end{array}$ \\
\hline 9 & $\begin{array}{l}\text { Stop if you meet resistance. Resi patient. } \\
\text { Seek further advice eg. senior nursing / } \\
\text { medical staff. } \\
\text { If there is difficulty removing a PICC line, it } \\
\text { may be helpful to warm arm. } \\
\text { Sedation may need to be prescribed }\end{array}$ & $\begin{array}{l}\text { Reduces the potential for venous spasm. } \\
\text { Sedation may help to decrease level of } \\
\text { anxiety for the patient and further } \\
\text { contribute to ease of removal. }\end{array}$ \\
\hline 10 & $\begin{array}{l}\text { Using sterile gauze apply pressure over the } \\
\text { exit site until bleeding stops ( } 5 \text { minutes). } \\
\text { May be longer if patient is on anti-coagulant } \\
\text { therapy. } \\
\text { Cover with IV pressure dot or occlusive } \\
\text { sterile dressing. }\end{array}$ & Prevents bleeding and air aspiration. \\
\hline 11 & $\begin{array}{l}\text { Inspect catheter ensuring it is complete with } \\
\text { no ragged edges. }\end{array}$ & $\begin{array}{l}\text { If it is not intact, tip may migrate to the } \\
\text { heart and pulmonary system and urgent } \\
\text { medical assistance will be required. }\end{array}$ \\
\hline 12 & $\begin{array}{l}\text { If infection of catheter is suspected - using } \\
\text { sterile scissors, cut off } 5 \mathrm{~cm} \text { at tip of catheter } \\
\text { and place in specimen container, send to } \\
\text { Laboratory for culture and sensitivity with } \\
\text { appropriate Pathology form. }\end{array}$ & $\begin{array}{l}\text { To identify source of infection and } \\
\text { appropriate treatment. }\end{array}$ \\
\hline 13 & Remove and dispose of equipment correctly. & \\
\hline 14 & Remove gloves and perform hand hygiene. & \\
\hline 15 & $\begin{array}{l}\text { Record procedure and any variances in the } \\
\text { patients clinical record. }\end{array}$ & : \\
\hline 16 & $\begin{array}{l}\text { Remove the dressing after } 24 \text { hours and } \\
\text { assess the site. }\end{array}$ & \\
\hline
\end{tabular}




\subsubsection{Raferences}

Auckland District frealth Board. (2004). Central Venous Catheter Management Paediatric. CVL Management Manual. Pp.1-40

Buswell, R., \& Beyea, S. (1998). Flushing Protocols for Tunneled Central Venous Catheters: An Integrative review of the Literature. The Online Journal of Knowledge Synthesis for Nursing Vol 5, Document Number 3

Canterbury DHB Radiology Services. (1998). PICC/Midline Catheters inserted in radiology. Doryan Mahalm, Jacqui Badger and Steve Cotterell.

Centers for Disease Control and Prevention (2002). Guidelines for the Prevention of Intravascular Catheter Related Infections. MMWR Vol 51. No. RR-10

Corrigan, A. M., Pelletier G., Alexander M. (Eds) (2000). Intravenous Nurse Specialty Care Curriculum for Intravenous Nursing. Philadelphia. Lippincott (Second Ed.)

Com C. (1983). The importance of syringe size when using implanted vascular access devices. Journal of Vascular Access Network. 3(1). Pp 11-18

Department of Health (2001). Guidelines for Preventing Infections associated with the insertion and maintenance of central venous catheters. Journal of Hospital Infection 47 (supplement) S47-S67

Dougherty L., Lamb. J. (1999.) Intravenous Therapy in Nursing Practice. Churchill Livingstone, Edinburgh

Infusion Nursing Standards of Practice (2000) Journal of Intravenous Nursing (supplement) Vol 2365

Joanna Briggs Institute of Evidence Based Nursing and Midwifery (2004) Parental Guidelines, Acute Care Manual.

Johnson \& Johnson Medical (1998). Peripherally Inserted Central Catheters, Patient Selection, Insertion and Maintenance. Division of Ethicon, Inc. Arlington.

Peripherally Inserted Central Catheters (1996). Workshop manual. HDC Corporation.

Terry, J. Baronowski, L., Lonsway, RA. Hedrick, C. (1995) Intravenous Therapy, Clinical Principals and Practice: Saunders, Pennsylvania.

Weinstein, S. M., (2000) Principles and Practice of Intravenous Therapy. Philadelphia. Lippincott $\left(7^{\text {th }}\right.$ Ed). 


\section{Appendix 5 - De-Cloting a PlC Catheter using the 'POP Techinigue'}

De-Clotting a PIC Catheter using the "POP Technique"

\section{Objective}

The "POP technique" is used to de-clot a PIC Catheter by sending a "shock wave" down the catheter. This is created by releasing the syringe plunger, causing it to 'Pop'.

The shock wave releases the thrombus (clot) from the catheter wall, allowing it to be aspirated up and out of the PIC catheter.

\section{Personnel Authorised to Perform this Procedure:}

Registered Nurses with a current IV certificate who

- have competence in dealing with CVAD's

- have the approval of the CCN of the area

\section{Guiding Principles}

- procedure performed using an aseptic technique to avoid contamination of the hub

- in order to avoid disconnection from the extension set, use a $10 \mathrm{ml}$ Luer lock syringe containing 1-2 mls normal saline, to perform the POP technique

- keep the syringe tip facing downwards with normal saline between the plunger and hub of the extension set

- perform pulsatile flushes with two $0.9 \%$ normal saline $(10 \mathrm{~m} / \mathrm{s})$ once thrombus removed and catheter is unblocked, to ensure catheter is completely clear of thrombus

\section{Equipment}

Disposable dressing pack

$310 \mathrm{ml}$ luer lock syringes

1 Sml syringe

$210 \mathrm{ml}$ ampoules $0.9 \%$ Normal saline

$15 \mathrm{ml}$ ampoule Heparinised Saline $50 \mathrm{iu} / 5 \mathrm{ml}$

1 needle free access port (luer plug/CLC 2000)

$1 \quad 1 \%$ Chlorhexidine/alcohol swab (Briemarpak)

1 Statlock with 12" tubing -PRN (Supply code MIV0?09)

2 Blunt Access cannulas

Non-sterile gloves 


\section{Action Steps}

1 Explanation given to patient

2 Fand wash. Don non-sterile gloves

3 Fill $10 \mathrm{ml}$ Luer Lock syringe with $1-2 \mathrm{mls}$ of Normal saline

4 Ensure extension set is clamped

5 Open out $1 \%$ chlorhexidine/alcohol swab (Briemarpak) to hold end of extension set in during procedure, using non-louch technique

6 Remove needle free access port from extension set.

7 Attach luer lock syringe with saline onto hub of extension set

8 Unclamp extension set

9 With syringe tip facing downwards, pull plunger fully back and then release. Release of the plunger may cause a "Pop" sound, sending a shock wave down the plunger

10 Keeping tip of syringe facing downwards, repeat this "pull back and release plunger", at 2 second intervals, up to a maximum of 30 attempts

11 At some stage, you should notice a free flow back of blood through the syringe due to it being unblocked with this "Pop" technique

12 Ensure extension set is clamped

13 Disconnect syringe

14 Place new, primed needle access port onto hub of extension set

15 Vigorously pulsatile flush twice with $10 \mathrm{ml}$ ampoules normal saline

16 "Lock" catheter with $5 \mathrm{mls}$ heparinised saline

17 Remove and dispose of equipment correctly

18 Wash hands

19 Document procedure and result in patient's clinical record

20 Contact nursing staff in DSA, Radiology department, for advice with this, as required

See attached article by Fetzer, S.J. (2004) "Safety and efficacy of the POP technique for restoring patency to occluded PIC catheters". Applied Nursing Research, Vol 17 (4) p297-300. 


\section{Maintenance and Care Plan}

Date:

Dressing Change:

Lear Plug Change:

External Catheter

Mesuremant:

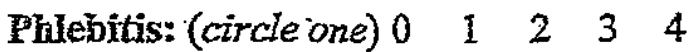

Otrer Complications:

Date:

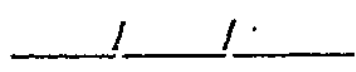

Dressing Chaüge:

Leur Flug Change:

Extermal Catheter

Measurement:

Phlebitis: (circle one) $0 \quad 1 \quad 2 \quad 3 \quad 4$

Other Complications:

Date:

Dressing Change:

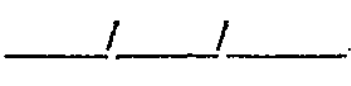

Tear-Plug Change:

Extemal Catheter

î̉easurement:

Phlebitis: (circle one) $0 \begin{array}{lllll}0 & 1 & 2 & 3 & 4\end{array}$

Other Complications:
Date:

Dressing Change:

Leur PIng Change:

Exítingl Catheter

Measurement:

Phlebitis: (circle one) $0 \quad 1 \quad 2 \quad 2 \quad 3 \quad 4$

Other Complications:

\section{Date:}

Dressing Change:

Lemr Plg Change:

Wxternal Catheter

Measurement:

Phlebitis: (circle one) $0 \quad 1 \quad 2 \quad 3 \quad 4$

Other Complications:

\section{Date:}

Dresting Change:

Leur Plag Change:

Extersal Catheter

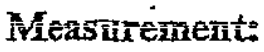

Phlebitis: (circle one) 0 1. $23 \quad 34$

Other Complications: 


\section{Central Venous Access Device Record}

Please circle one

1. INSERTION

Type

PICC

Hickman Catheter

Implanted Port

Reason for Insertion Poor venous access

Continuous infusion

Chemo type

Date of Insertion

Complications of Insertion

Haematoma

Phiebitis

Allergic reaction

Catheter position

Left

Right

. External catheter measurement

Patient Education

Yes: No

'Person attending flush

Nurse - Patient

Relative

Nurse Maude

Referred to District Nurse

Yes

No

Name:

Signature:

Date:

\section{COMPLICATIONS}

\section{Date}

Action

Difficulty with flushing

Haematoma

Phlebitis

Haemorrhage

Thrombus

Name:

Signature:

Date:

3. REMOVAL

Date of Removal

Reason for removal Routine Disease progression Complications

Comments:

Name:

Signature:

Date: 


\section{Central Venous Access Device Record Cancer Type \\ Cancer Site \\ Metastases}

Please circle one

1. INSERTION

Type PICC Hickman Catheter Implanted Port

Reason for Insertion Poor venous access Continuous chemo infusion

Date of Insertion

Intermittent chemo Other-please state:

Complications of Insertion Haematoma Phlebitis Allergic reaction

Catheter position Left Right

External catheter measurement

Paţienté Educat́ion

Yes No

Person attending flush Nurse Patient Relative Nurse Maude

Referred to District Nurse Yes No

Name: $\quad$ Signature:

Date:

\section{COMPLICATIONS}

Date

Action

Difficulty with flushing

Catheter Occlusion

Phlebitis

Infection

Thrombus

PICC Migration

Name:

Signature:

Date:

\section{REMOVAL}

Date of Removal

$\begin{array}{ll}\text { Reason for removal Routine Disease progression } & \text { PICC complications } \\ \text { Chemo complications }\end{array}$

Comments:

Name:

Signature:

Date: 
Appenax $y$ - Camrerbury ystract Heanth board Approval for use of its documents within thin thesis

\section{Canterbury \\ District Health Board \\ Te Poari Hauora ō Waitaha}

General Manąger, Medical \& Surgical Division

Lower Ground Floor, Parkside West

Christchurch Hospital, Christchurch
Tel:

Fax:

E-Mail:
(03) 3640036

(03) 3640453

mark.leggett@cdhb.govt.nz

15 February 2008

Mary Fairhall

46 Kotara Street

Riccarton

Christchurch 8041

Dear Mary

\section{Research Project}

Thank you for your letter of 21 January 2008, in regard to your research project: Peripherally inserted Central Catheter Related Complications Amongst Oncology Patients.

As sought, approval is given to include the following forms, sheets and booklets in the appendix of your thesis document:

* PICC/Midline Patent Information form

* Canterbury DHB 'Guidelines/protocols for accessing PICC

- PICC Maintenance and Care Plan

- Central Venous Access Device Record

- A guide to your PICC/Midline: CLC 200 - saline lock

- Peripherally inserted central catheter PICC Patient information Booklet: Insertion Procedure.

Please accept my apologies for the delay in advising this approval.

Wishing you all the best for the masters study you are undertaking; it is good to see staif furthering their knowledge in areas of interest.

rours sincerely.

Mark Leggett

General Manager

Medical \& Surgical Division

Copy to Robyn Hulme - Acting Director of Nursing

Doryan Mahlm - CCN, DSA, Radiology Department

Bernie Fitzharris- Clinical Director, Oncology Department 